Prepared in cooperation with:

Bureau of Land Managment

City of Gunnison

Colorado River Water Conservation District

Crested Butte South Metropolitan District

Gunnison County

Hinsdale County
Mount Crested Butte Water and Sanitation District National Park Service Town of Crested Butte Upper Gunnison River Water Conservancy District Western State College

\title{
Comparison of Water Years 2004-05 and Historical Water- Quality Data, Upper Gunnison River Basin, Colorado
}

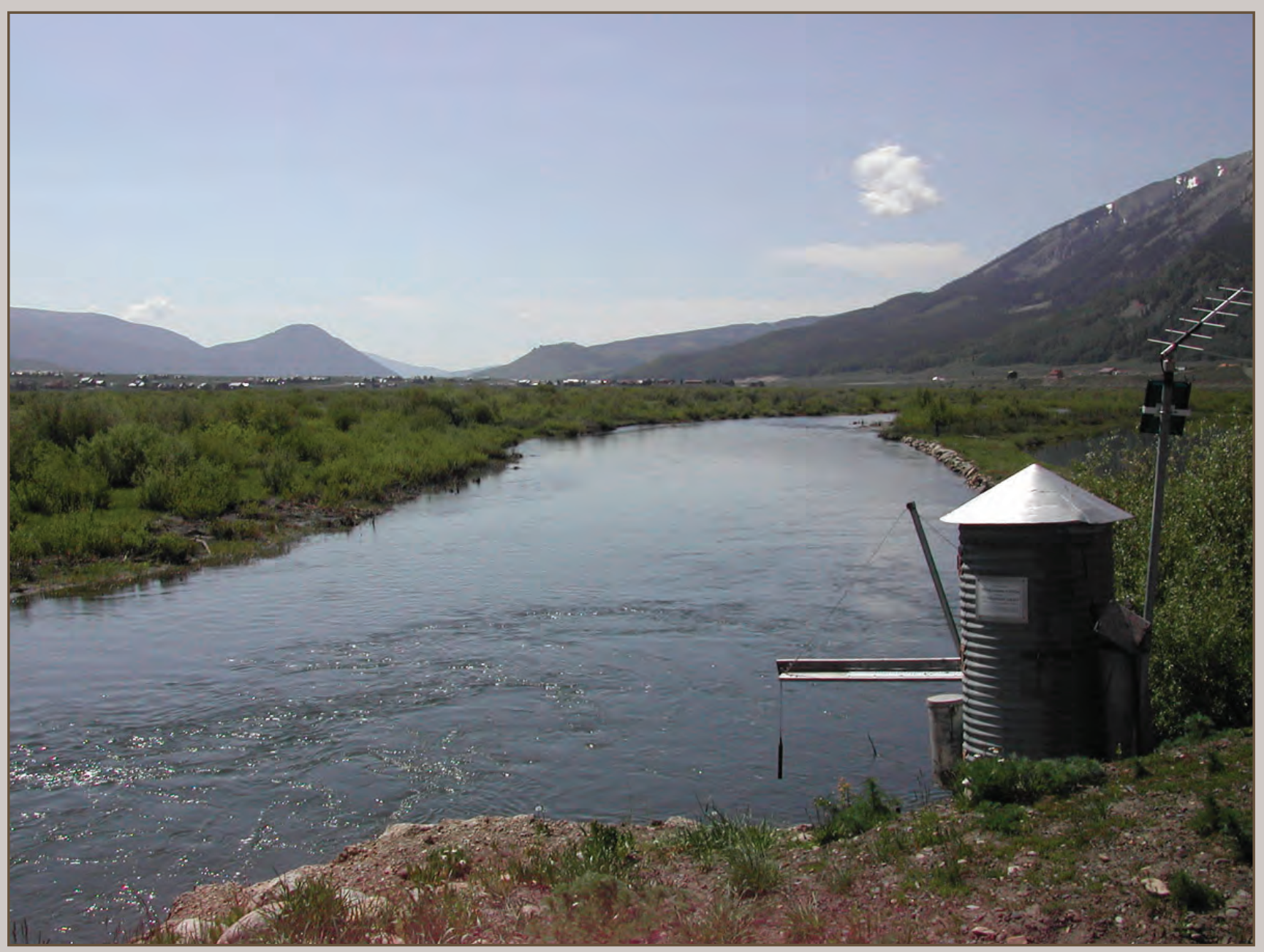

Data Series Report 331

U.S. Department of the Interior

U.S. Geological Survey 


\section{FRONT COVER}

Slate River near Crested Butte, Colorado.

Photograph by David Hartle, U.S. Geological Survey. 


\section{Comparison of Water Years 2004-05 and Historical Water-Quality Data, Upper Gunnison River Basin, Colorado}

By Norman E. Spahr, David M. Hartle, and Paul Diaz

Prepared in cooperation with:

Bureau of Land Management

City of Gunnison

Colorado River Water Conservation District

Crested Butte South Metropolitan District

Gunnison County

Hinsdale County

Mount Crested Butte Water and Sanitation District

National Park Service

Town of Crested Butte

Upper Gunnison River Water Conservancy District

Western State College

Data Series Report 331 


\section{U.S. Department of the Interior DIRK KEMPTHORNE, Secretary}

\section{U.S. Geological Survey \\ Mark D. Myers, Director}

\section{U.S. Geological Survey, Reston, Virginia: 2008}

For product and ordering information:

World Wide Web: http://www.usgs.gov/pubprod

Telephone: 1-888-ASK-USGS

For more information on the USGS — the Federal source for science about the Earth, its natural and living resources, natural hazards, and the environment:

World Wide Web: http://www.usgs.gov

Telephone: 1-888-ASK-USGS

Any use of trade, product, or firm names is for descriptive purposes only and does not imply endorsement by the U.S. Government.

Although this report is in the public domain, permission must be secured from the individual copyright owners to reproduce any copyrighted materials contained within this report.

Suggested citation:

Spahr, N.E., Hartle, D.M, and Diaz, Paul, 2008, Comparison of water years 2004-05 and historical water-quality data, upper Gunnison River Basin, Colorado: U.S. Geological Survey Data Series Report 331, 54 p. 


\section{Contents}

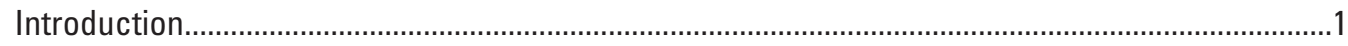

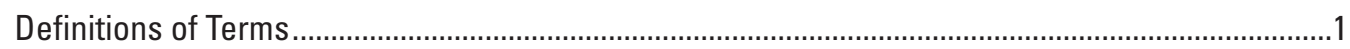

385224106590100 Coal Creek above Mouth at Crested Butte, CO ....................................................6

385325106581200 Washington Gulch below Woods Creek at Mount Crested Butte, CO ....................10

09111500 Slate River near Crested Butte, CO ....................................................................................13

384950106544200 East River above Slate River near Crested Butte, CO..........................................17

09112200 East River below Cement Creek, CO ................................................................................20

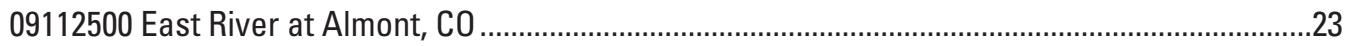

09113500 Ohio Creek near Baldwin ..................................................................................................26

09113980 Ohio Creek above Mouth, near Gunnison, CO ....................................................................29

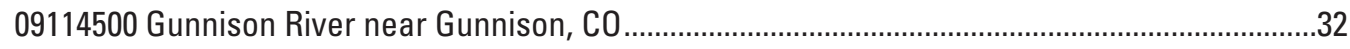

383126106475600 Tomichi Creek below Cochetopa Creek, CO ........................................................35

09119000 Tomichi Creek at Gunnison, CO ...................................................................................39

383103106594200 Gunnison River at County Road 32 below Gunnison, CO .....................................43

380133107190000 Henson Creek at Mouth at Lake City ..................................................................47

380233107180701 Lake Fork Gunnison River near Lake City ...........................................................50

385229106583100 Crested Butte Wastewater Treatment Plant ...........................................................53

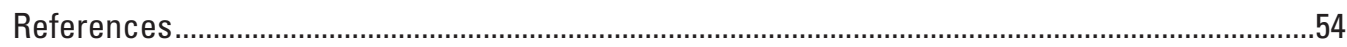

\section{Figures}

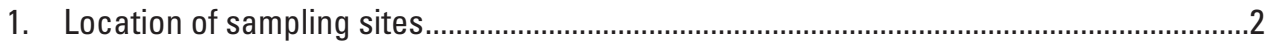

2-30. Graphs showing:

2. Time distribution and streamflow of water-quality samples for Coal Creek above Mouth at Crested Butte .......................................................................6

3. Distribution of selected water-quality constituents relative to time for Coal Creek above Mouth at Crested Butte ..................................................................7

4. Time distribution and streamflow of water-quality samples for Washington Gulch below Woods Creek at Mount Crested Butte.......................................................10

5. Distribution of selected water-quality constituents relative to time for Washington Gulch below Woods Creek at Mount Crested Butte...

6. Daily mean streamflow and time distribution of water-quality samples for Slate River near Crested Butte.

7. Annual streamflow and distribution of selected water-quality constituents relative to time for Slate River near Crested Butte

8. Time distribution and streamflow of water-quality samples for East River above Slate River near Crested Butte

9. Distribution of selected water-quality constituents relative to time for East River above Slate River near Crested Butte.

10. Daily mean streamflow and time distribution of water-quality samples for East River below Cement Creek....

11. Annual streamflow and distribution of selected water-quality constituents relative to time for East River below Cement Creek. 
2-30. Graphs showing:-Continued

12. Daily mean streamflow and time distribution of water-quality samples for East River at Almont.

13. Annual streamflow and distribution of selected water-quality constituents relative to time for East River at Almont.

14. Time distribution and streamflow of water-quality samples for for Ohio Creek near Baldwin.

15. Distribution of selected water-quality constituents relative to time for Ohio Creek near Baldwin.

16. Daily mean streamflow and time distribution of water-quality samples for Ohio Creek above mouth near Gunnison..

17. Annual streamflow and distribution of selected water-quality constituents relative to time for Ohio Creek above mouth near Gunnison ......

18. Daily mean streamflow and time distribution of water-quality samples for Gunnison River at Gunnison.

19. Annual streamflow and distribution of selected water-quality constituents relative to time for Gunnison River at Gunnison

20. Time distribution and streamflow of water-quality samples for Tomichi Creek below Cochetopa Creek.

21. Distribution of selected water-quality constituents relative to time for Tomichi Creek below Cochetopa Creek.

22. Streamflow and time distribution of water-quality samples for Tomichi Creek at Gunnison

23. Annual streamflow and distribution of selected water-quality constituents relative to time for Tomichi Creek at Gunnison.

24. Time distribution and streamflow of water-quality samples for Gunnison River at County Road 32 below Gunnison.

25. Distribution of selected water-quality constituents relative to time for Gunnison River at County Road 32 below Gunnison

26. Time distribution and streamflow of water-quality samples for Henson Creek at mouth at Lake City .

27. Distribution of selected water-quality constituents relative to time for Henson Creek at mouth at Lake City.

28. Time distribution and streamflow of water-quality samples for Lake Fork Gunnison River near Lake City.

29. Distribution of selected water-quality constituents relative to time for Lake Fork Gunnison River near Lake City

30. Distribution of total phosphorus samples relative to time for Crested Butte Wastewater Treatment Plant.

\section{Tables}

1. Summary of individual station results.

2-15. Summary of measured constituents and properties for:

2. Coal Creek above Mouth at Crested Butte station 385224106590100 ..........................

3. Washington Gulch below Woods Creek station 385325106581200 ….......................12

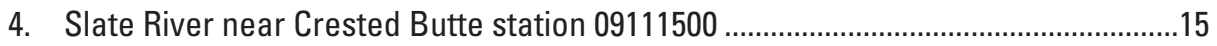

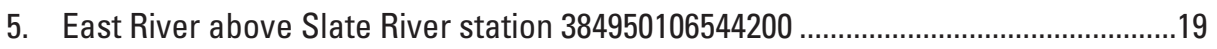


2-15. Summary of measured constituents and properties for:-Continued

6. East River below Cement Creek station 09112200 ......................................................22

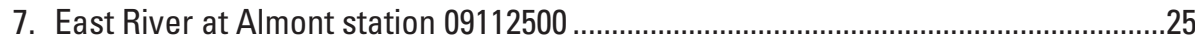

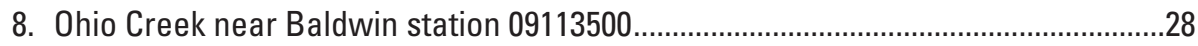

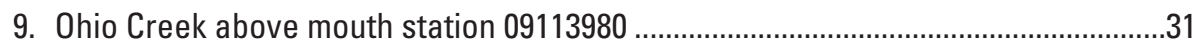

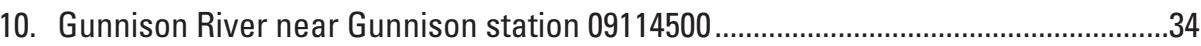

11. Tomichi Creek below Cochetopa Creek station $383126106475600 \ldots \ldots \ldots \ldots \ldots \ldots \ldots \ldots \ldots \ldots . . .37$

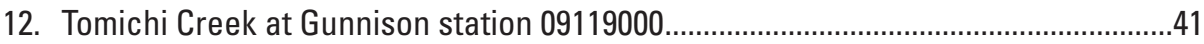

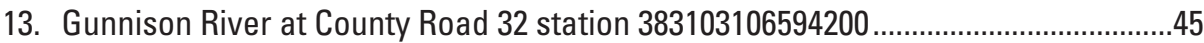

14. Henson Creek at Mouth at Lake City station 380133107190000 ................................49

15. Lake Fork Gunnison River near Lake City station 380233107180701 .........................52

16. Summary of total phosphorus for Crested Butte Wastewater Treatment

Plant site 385229106583100 . .53

\section{Conversion Factors and Datums}

\begin{tabular}{|c|c|c|}
\hline Multiply & By & To obtain \\
\hline \multicolumn{3}{|c|}{ Length } \\
\hline inch & 2.54 & centimeter $(\mathrm{cm})$ \\
\hline inch & 25.4 & millimeter (mm) \\
\hline foot $(\mathrm{ft})$ & 0.3048 & meter $(\mathrm{m})$ \\
\hline mile (mi) & 1.609 & kilometer $(\mathrm{km})$ \\
\hline \multicolumn{3}{|c|}{ Area } \\
\hline acre & 0.4047 & hectare (ha) \\
\hline square foot $\left(\mathrm{ft}^{2}\right)$ & 0.09290 & square meter $\left(\mathrm{m}^{2}\right)$ \\
\hline square mile $\left(\mathrm{mi}^{2}\right)$ & 259.0 & hectare (ha) \\
\hline square mile $\left(\mathrm{mi}^{2}\right)$ & 2.590 & square kilometer $\left(\mathrm{km}^{2}\right)$ \\
\hline \multicolumn{3}{|c|}{ Volume } \\
\hline gallon (gal) & 3.785 & liter $(\mathrm{L})$ \\
\hline gallon (gal) & 0.003785 & cubic meter $\left(\mathrm{m}^{3}\right)$ \\
\hline cubic foot $\left(\mathrm{ft}^{3}\right)$ & 0.02832 & cubic meter $\left(\mathrm{m}^{3}\right)$ \\
\hline acre-foot (acre-ft) & 1,233 & cubic meter $\left(\mathrm{m}^{3}\right)$ \\
\hline \multicolumn{3}{|c|}{ Flow rate } \\
\hline acre-foot per day (acre-ft/d) & 0.01427 & cubic meter per second $\left(\mathrm{m}^{3} / \mathrm{s}\right)$ \\
\hline cubic foot per second $\left(\mathrm{ft}^{3} / \mathrm{s}\right)$ & 0.02832 & cubic meter per second $\left(\mathrm{m}^{3} / \mathrm{s}\right)$ \\
\hline
\end{tabular}

Temperature in degrees Celsius $\left({ }^{\circ} \mathrm{C}\right)$ may be converted to degrees Fahrenheit $\left({ }^{\circ} \mathrm{F}\right)$ as follows:

$$
{ }^{\circ} \mathrm{F}=\left(1.8 \times^{\circ} \mathrm{C}\right)+32
$$

Vertical coordinate information is referenced to the North American Vertical Datum of 1988 (NAVD 88).

Horizontal coordinate information is referenced to the North American Datum of 1983 (NAD 83).

Altitude, as used in this report, refers to distance above the vertical datum.

Specific conductance is given in microsiemens per centimeter at 25 degrees Celsius $(\mu \mathrm{S} / \mathrm{cm}$ at $\left.25^{\circ} \mathrm{C}\right)$.

Concentrations of chemical constituents in water are given either in milligrams per liter (mg/L) or micrograms per liter $(\mu \mathrm{g} / \mathrm{L})$. 



\title{
Comparison of Water Years 2004-05 and Historical Water- Quality Data, Upper Gunnison River Basin, Colorado
}

\author{
By Norman E. Spahr, David M. Hartle, and Paul Diaz
}

\section{Introduction}

Population growth and changes in land use have the potential to affect water quality and quantity in the upper Gunnison River Basin. In 1995, the U.S. Geological Survey (USGS), in cooperation with the Bureau of Land Management, City of Gunnison, Colorado River Water Conservation District, Crested Butte South Metropolitan District, Gunnison County, Hinsdale County, Mount Crested Butte Water and Sanitation District, National Park Service, Town of Crested Butte, Upper Gunnison River Water Conservancy District, and Western State College, established a water-quality monitoring program in the upper Gunnison River Basin to characterize current water-quality conditions and to assess the effects of increased urban development and other land-use changes on water quality. The monitoring network has evolved into two groups of stations-stations that are considered long term and stations that are considered rotational. The long-term stations are monitored to assist in defining temporal changes in water quality (how conditions may change over time). The rotational stations are monitored to assist in the spatial definition of water-quality conditions (how conditions differ throughout the basin) and to address local and short-term concerns. Some stations in the rotational group were changed beginning in water year 2007. Annual summaries of the waterquality data from the monitoring network provide a point of reference for discussions regarding water-quality monitoring in the upper Gunnison River Basin.

This summary includes data collected during water years 2004 and 2005. The introduction provides a map of the sampling sites, definitions of terms, and a one-page summary of selected water-quality conditions at the network stations. The remainder of the summary is organized around the data collected at individual stations. Data collected during water years 2004 and 2005 are compared to historical data, State water-quality standards, and Federal water-quality guidelines. Data were collected following USGS protocols (U.S. Geological Survey, variously dated).

\section{Definitions of Terms}

Constituent results for which the State of Colorado has adopted a "chronic instream standard," or in the absence of that, for which the U.S. Environmental Protection Agency (USEPA) has published a "recommended level," are reported in terms of "concern levels" as defined below:

Low Concern: The majority (85th percentile) of samples for a given site are below one-half the instream standard or recommended level. For example, if a constituent had a standard of 200 and the 85th percentile of the data was less than 100, it would be listed as low concern.

Concern: The 85th percentile of the data is between the instream standard and one-half the standard. This means that 15 percent or more of the data for a given site are nearing or are greater than the standard.

High Concern: The 85th percentile of the data is above the instream standard or recommended level.

Cannot Classify: The data cannot be placed into one of the three concern levels due to detection limits, no instream standard, or lack of samples.

The geometric mean is used for Escherichia coli rather than the 85th percentile.

If a measured constituent is not listed with a concern level, it can be assumed that it is likely a low concern. 


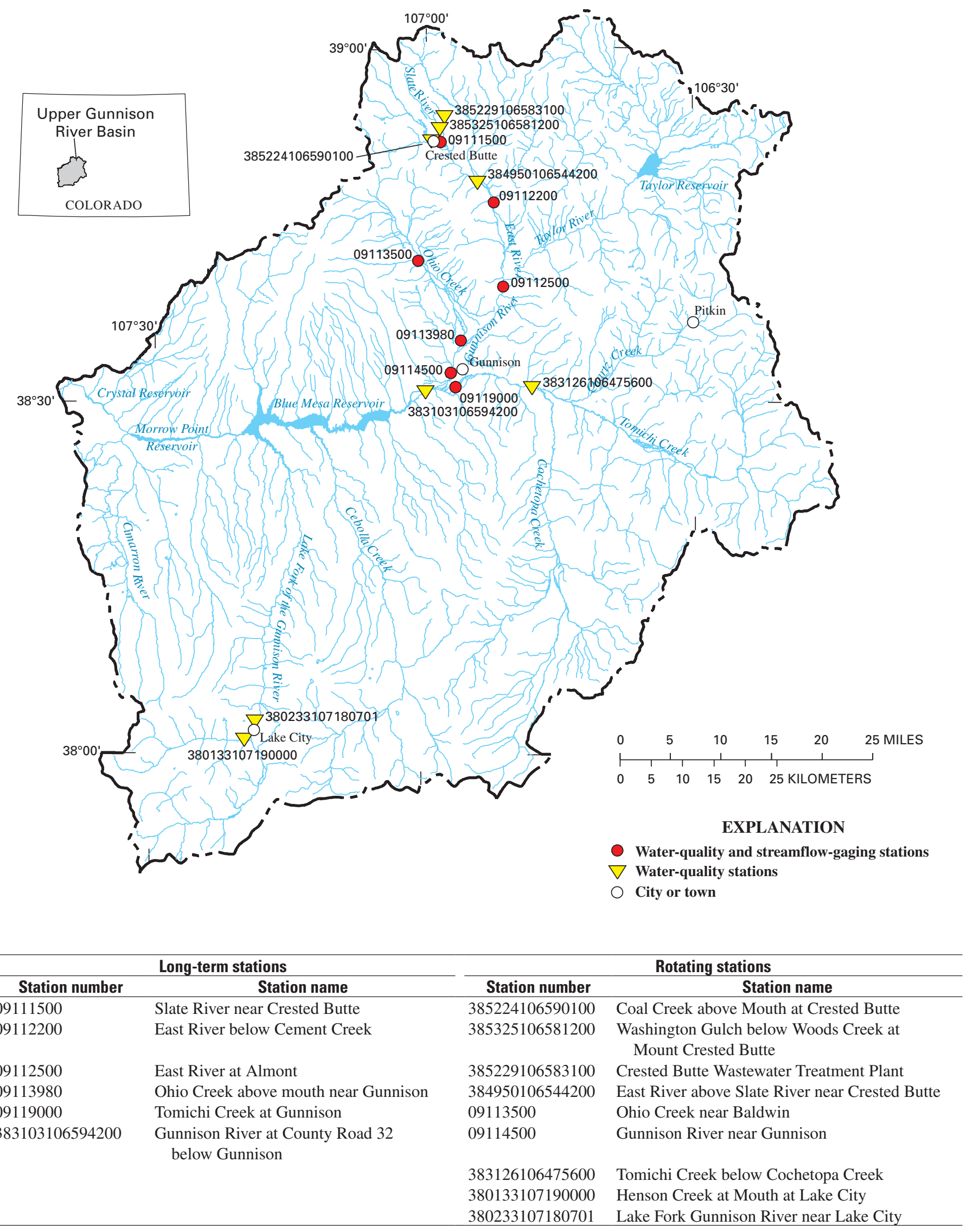

Figure 1. Location of sampling sites. 
The concern levels are consistent with the methods used by the State of Colorado to assess whether stream water-quality standards are being attained. The following is from the Unified Assessment Methodology, Water Quality Control Division, accessed April 2004 at http://www.cdphe.state.co.us/wq/Assessment/assessment_practices_and_methods.htm:

"1. Attainment of chronic chemical standards, in both lotic (streams and rivers) and limnic (lakes and reservoirs) systems, is based upon the 85th percentile of the ranked data, except as otherwise noted below. Percentile values are calculated by ranking individual data points in order of magnitude. Hardness-based metal standards are evaluated by comparing the 85th percentile against the assigned hardness based equation using either the mean hardness at low flow or, when available, paired hardness and flow data. Total recoverable iron is evaluated against the median value, or the 50th percentile. Dissolved oxygen is evaluated at the 15th percentile. Minima $\mathrm{pH}$ is evaluated against the 15 th percentile, maxima at the 85th.

2. Acute standards are evaluated by comparison of raw values against the standard.

3. Sample data that are below detection limits will, in general, be treated as zeroes for assessment of attainment of chronic standards.

4. Attainment of coliform standards is assessed using the geometric mean. Notwithstanding the criterion at item 3 above, coliform data which is reported as less than detect will be treated as a value of one to allow calculation of a geometric mean."

State standards for dissolved oxygen are based on minima, and for $\mathrm{pH}$, they are based on a range. The concern levels are defined as follows:

\section{Dissolved Oxygen Concern Levels:}

Low concern: above $7 \mathrm{mg} / \mathrm{L}$

Concern: between 6 and $7 \mathrm{mg} / \mathrm{L}$

High concern: below $6 \mathrm{mg} / \mathrm{L}$ (below State instream standard)

\section{pH Concern Levels:}

Low concern: between 7 and 8.5

Concern: between 6.5 and 7 or 8.5 and 9.0

High concern: outside the range of 6.5-9.0 (outside of State standards)

Total-Phosphorus: Concern levels are based on the USEPA recommendations of 0.1 milligram per liter $(\mathrm{mg} / \mathrm{L})$ for water not directly flowing into a lake or reservoir (U.S. Environmental Protection Agency, 1986).

Hardness Definitions: (based on Durfor and Besker, 1964, p. 27, as cited in Hem, 1992):

Soft: $0-60 \mathrm{mg} / \mathrm{L}$

Moderately hard: $61-120 \mathrm{mg} / \mathrm{L}$

Hard: $121-180 \mathrm{mg} / \mathrm{L}$

Very hard: greater than $180 \mathrm{mg} / \mathrm{L}$

HUC: Hydrologic Unit Code—A geographic area representing part or all of a surface drainage basin or distinct hydrologic feature. Each hydrologic unit is identified by an 8-digit number.

Censored Value: A value reported as less than a laboratory reporting level, for example $<0.05$.

Laboratory Reporting Level (LRL) generally is equal to twice the yearly determined long-term method detection level (LT-MDL). The LRL controls false-negative error. The probability of falsely reporting a nondetection for a sample that contained an analyte at a concentration equal to or greater than the LRL is predicted to be less than or equal to 1 percent. The value of the LRL will be reported with a "less than" $(<)$ remark code for samples in which the analyte was not detected. The National Water Quality Laboratory collects quality-control data from selected analytical methods on a continuing basis to determine LT-MDLs and to establish LRLs. These values are reevaluated annually based on the most current quality-control data and therefore may change. (Note: Previously, the LRL has been called the nondetection value or NDV—a term which is no longer used.) 
Stream Segment: (from Colorado Department of Public Health and Environment Water Quality Control Commission Regulation number 31)

"(a) For purposes of adopting site-specific classifications and water quality standards, the streams and other surface water bodies shall be identified according to river basin and/or subbasin and specific water segments.

(b) Segments may constitute a specified stretch of a river mainstem, a specific tributary, a specific lake or reservoir, or a generally defined grouping of waters within the basin (e.g., a specific mainstem segment and all tributaries flowing into that mainstem segment.

(c) Segments shall generally be delineated according to the points at which the use, physical characteristics or water quality characteristics of a watercourse are determined to change significantly enough to require a change in use classifications and/or water quality standards. In many cases, such transition points can be specifically identified from available water quality data. In other cases, however, the delineation of segments shall be based upon best judgments of where instream changes in uses, physical characteristics or water quality occur, based upon upstream and downstream data."

Water supply standard refers to waters with a water-supply classification, Colorado Department of Public Health and Environment, Water Quality Control Commission Regulation number 31.11 (6).

303 (d): (from Colorado Department of Public Health and Environment, Colorado's Section 303(d) Listing Methodology, September 9, 2003, accessed at http://www.cdphe.state.co.us/op/wqcc/SpecialTopics/303(d)/303dtmdlpro.html)

"Section 303(d) of the federal Clean Water Act requires states to identify waters where effluent limitations mandated by Section 301(b)(1)(A) and Section 301(b)(1)(B) are not stringent enough to attain water quality standards. These waters are compiled into the Section 303(d) list of impaired waters. The Colorado Section 303(d) List identifies those water bodies, which are impaired by one or more pollutants."

Dissolved and Total: Constituent concentrations listed in the accompanying figures and tables refer to dissolved concentrations unless specifically stated otherwise.

Trend analyses were performed using the seasonal Kendall test (Helsel and Hirsch, 1993) using flow-adjusted concentrations. This analysis measures the monotonic relation between constituent concentration and time. Because the analysis is rank-based, it is resistant to the effects of small sample size, censored data, and non-normal population distributions. A trend was determined to be present when the p-value of the statistical test was less than the decision level (alpha-level) of 0.05. Trends identified as "up" indicate that the flow-adjusted concentrations increased more often than decreased over time. Trends identified as "down" indicate that the flow-adjusted concentration decreased more often than increased over time. A trend identified as "none" indicates there is insufficient evidence for rejection of the null hypothesis, which states that no relation between concentration and time exists. 
Table 1. Summary of individual station results.

[Alk, Alkalinity; Ca, Calcium; Cd, Cadmium; Cl, chloride; Conductance, specific conductance; Al, aluminum; Cu, copper; DO, dissolved oxygen; E. coli, Escherichia coli; Fe, iron; Mg, magnesium; $\mathrm{Mn}$, manganese; Ortho, orthophosphate; $\mathrm{SO}_{4}$, sulfate; $\mathrm{Zn}$, zinc]

\begin{tabular}{|c|c|c|c|c|c|}
\hline $\begin{array}{l}\text { Station name and number } \\
\text { from figure } 1 \text { and table } 1\end{array}$ & Nutrients & $\begin{array}{c}\text { Metals/ } \\
\text { trace elements }\end{array}$ & $\begin{array}{c}\mathrm{pH}, \\
\text { dissolved oxygen }\end{array}$ & E. coli & Trend \\
\hline $\begin{array}{l}\text { Coal Creek above Mouth at } \\
\text { Crested Butte 3852241065901002 }\end{array}$ & Low concern & $\begin{array}{l}\text { High concern: } \mathrm{Cd}, \mathrm{Zn} \\
\text { Concern: } \mathrm{Al}, \mathrm{Cu}\end{array}$ & Low concern & Low concern & $\begin{array}{l}\text { None: } \mathrm{DO}, \mathrm{pH} \text {, conductance, calcium, magnesium, ammonia } \\
\text { plus organic, nitrate, total phosphorus, } \mathrm{Al}, \mathrm{Cd}, \mathrm{Cu}, \mathrm{Fe}, \mathrm{Mn}, \mathrm{Zn} \text {, } \\
\text { suspended sediment, } E \text {. coli }\end{array}$ \\
\hline $\begin{array}{l}\text { Washington Gulch below Woods } \\
\text { Creek at Mount Crested Butte } \\
385325106581200\end{array}$ & $\begin{array}{l}\text { High concern: } \\
\text { total phosphorus }\end{array}$ & No data & Low concern & Low concern & Insufficient data \\
\hline $\begin{array}{l}\text { Slate River near Crested Butte } \\
\quad 09111500\end{array}$ & $\begin{array}{l}\text { Concern: } \\
\quad \text { total phosphorus }\end{array}$ & Concern: $\mathrm{Zn}$ & Low concern & Low concern & $\begin{array}{l}\text { Down: DO, ammonia plus organic } \\
\text { Up: total phosphorus } \\
\text { None: } \mathrm{pH} \text {, conductance, hardness, ammonia, nitrate, orthophosphate, } \\
\text { Fe, } \mathrm{Zn} \text {, sediment }\end{array}$ \\
\hline $\begin{array}{l}\text { East River above Slate River near } \\
\quad \text { Crested Butte } 384950106544200\end{array}$ & Low concern & No data & Low concern & Low concern & None: $\mathrm{DO}, \mathrm{pH}$, conductance, nitrate \\
\hline $\begin{array}{l}\text { East River below Cement Creek } \\
\quad 09112200\end{array}$ & Low concern & Low concern & Low concern & Low concern & $\begin{array}{l}\text { Up: chloride } \\
\text { None: } \mathrm{DO}, \mathrm{pH} \text {, conductance, alkalinity, sulfate, nitrate, sediment }\end{array}$ \\
\hline East River at Almont 09112500 & Low concern & No data & Low concern & Low concern & $\begin{array}{l}\text { Down: DO, nitrate } \\
\text { Up: conductance } \\
\text { None: } \mathrm{pH}\end{array}$ \\
\hline Ohio Creek near Baldwin 09113500 & $\begin{array}{l}\text { Concern: } \\
\quad \text { total phosphorus }\end{array}$ & Low concern: Fe & Low concern & Low concern & Insufficient data \\
\hline $\begin{array}{l}\text { Ohio Creek above Mouth near } \\
\text { Gunnison } 09113980\end{array}$ & $\begin{array}{l}\text { Concern: } \\
\quad \text { total phosphorus }\end{array}$ & No data & Low concern & Low concern & None: DO, pH, conductance, orthophosphate, total phosphorus \\
\hline $\begin{array}{l}\text { Gunnison River near Gunnison } \\
09114500\end{array}$ & Low concern & No data & Low concern & Low concern & $\begin{array}{l}\text { Up: conductance } \\
\text { None: } \mathrm{DO}, \mathrm{pH}\end{array}$ \\
\hline $\begin{array}{l}\text { Tomichi Creek below Cochetopa } \\
\text { Creek } 383126106475600\end{array}$ & $\begin{array}{l}\text { Concern: } \\
\quad \text { total phosphorus }{ }^{1}\end{array}$ & Low concern & Low concern & Low concern & $\begin{array}{l}\text { Down: orthophosphate, total phosphorus } \\
\text { None: DO, pH, conductance, hardness, } \mathrm{Ca}, \mathrm{Mg} \text {, ammonia plus } \\
\text { organic, FE, Mn, E. coli, sediment }\end{array}$ \\
\hline $\begin{array}{l}\text { Tomichi Creek at Gunnison } \\
09119000\end{array}$ & $\begin{array}{l}\text { Concern: } \\
\quad \text { total phosphorus }\end{array}$ & Low concern & Low concern & Low concern & $\begin{array}{l}\text { Down: orthophosphate } \\
\text { None: DO, pH,, conductance, total phosphorus }\end{array}$ \\
\hline $\begin{array}{l}\text { Gunnison River at County } \\
\text { Road } 32 \text { below Gunnison } \\
383103106594200\end{array}$ & $\begin{array}{l}\text { Concern: } \\
\quad \text { total phosphorus }\end{array}$ & Low concern & Low concern & Low concern & $\begin{array}{l}\text { Up: conductance, hardness, } \mathrm{Ca}, \mathrm{Mg} \\
\text { None: } \mathrm{DO}, \mathrm{pH} \text {, total phosphorus, manganese }\end{array}$ \\
\hline $\begin{array}{l}\text { Henson Creek at Mouth at Lake City } \\
380133107190000\end{array}$ & Low concern & Concern: Al, Zn & Low concern & No data & Insufficient data \\
\hline $\begin{array}{l}\text { Lake Fork Gunnison River near Lake } \\
\text { City } 380233107180701\end{array}$ & Low concern & Concern: Al & Low concern & Low concern & Insufficient data \\
\hline
\end{tabular}

${ }^{1}$ Total phosphorus concern levels are based on the USEPA recommendations of 0.1 milligram per liter (mg/L) for water not directly flowing into a lake or reservoir (U.S. Environmental Protection Agency, 1986).
${ }^{2}$ This station name was changed from Coal Creek above Mouth near Crested Butte, CO, to Coal Creek above Mouth at Crested Butte, CO, in 2003. 


\section{Coal Creek above Mouth at Crested Butte, C0'1}

Current Reason for Inclusion: To collect baseline data, characterize effects from mining, and facilitate remediation of the affected surface waters. Part of the rotational group of stations.

\section{General Station Information:}

Location: Pedestrian bridge on Butte Avenue in Crested Butte.

Station Type: USGS water quality

Latitude: 385224

Longitude: 1065901
Drainage area: Not determined Stream segment: 12
HUC: 14020001

\section{USGS Data Summary:}

Period of Record:

Water quality: November 2000-September 2005

General Chemistry:

Water type: Insufficient major-ion data

Hardness: Moderately hard

Dissolved oxygen: Low concern

Nutrients: Low concern

E. coli: Low concern

Trace Elements/Metals:

High concern: Cadmium and zinc

Concern: Aluminum and copper

Other constituents of concern: None

${ }^{1}$ This station name was changed from Coal Creek above Mouth near Crested Butte, CO, to Coal Creek above Mouth at Crested Butte, CO, in 2003.

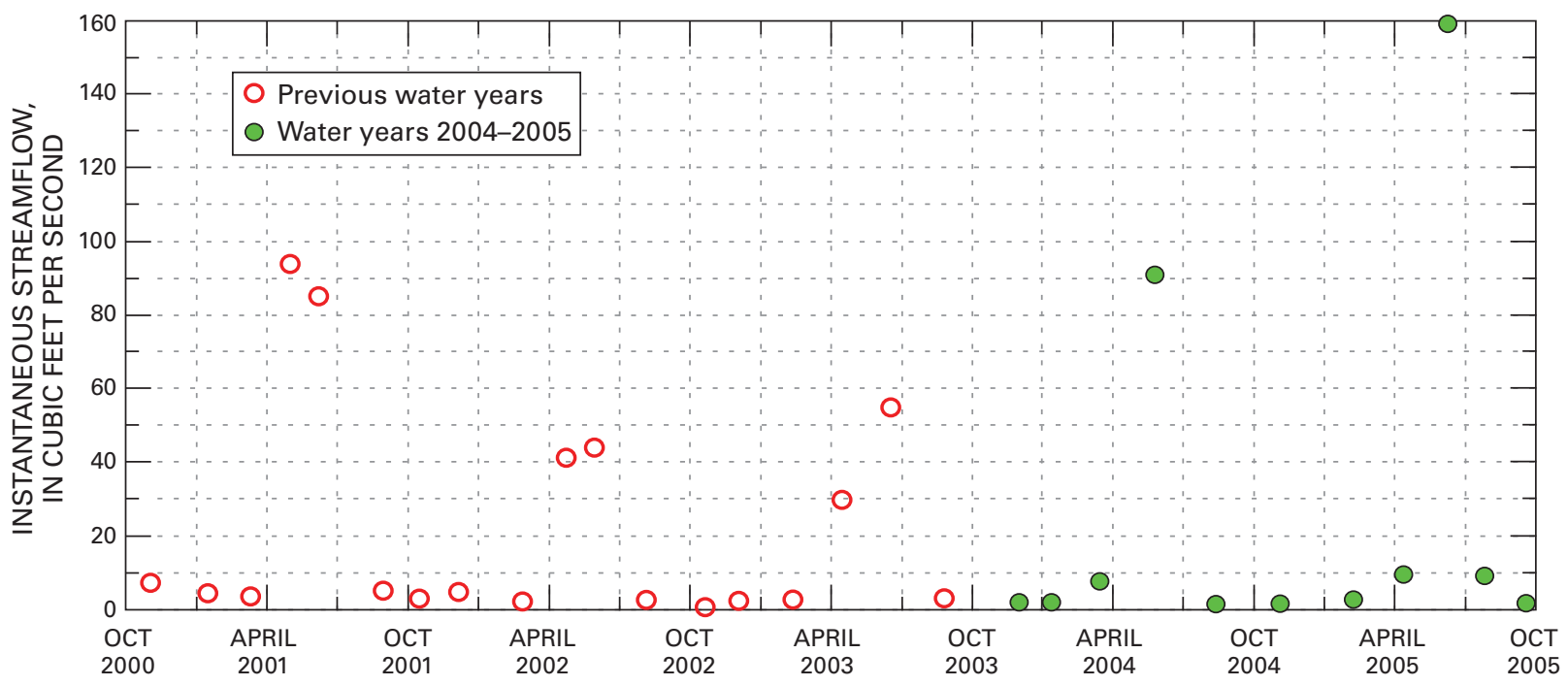

Figure 2. Time distribution and streamflow of water-quality samples for Coal Creek above Mouth at Crested Butte. 

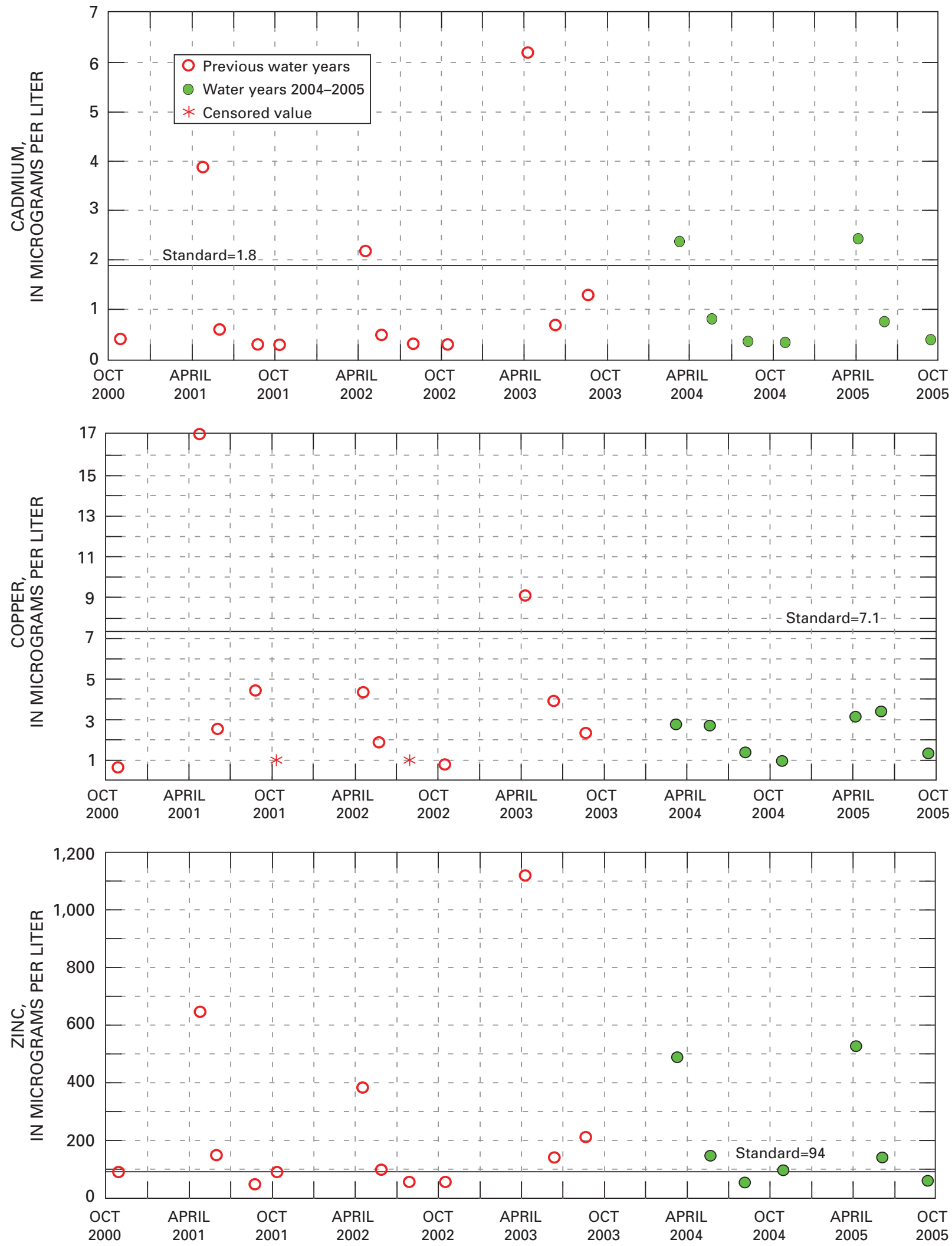

Figure 3. Distribution of selected water-quality constituents relative to time for Coal Creek above Mouth at Crested Butte. 
Table 2. Summary of measured constituents and properties for Coal Creek above Mouth at Crested Butte station 385224106590100.

[This station name was changed from Coal Creek above Mouth near Crested Butte, CO, to Coal Creek above Mouth at Crested Butte, CO, in 2003. mg/L, milligrams per liter; $\mu \mathrm{S} / \mathrm{cm}$, microsiemens per centimeter at $25^{\circ}$ Celsius; $\mu \mathrm{g} / \mathrm{L}$, micrograms per liter; ${ }^{\circ} \mathrm{C}$, degrees Celsius; $\mathrm{CaCO}_{3}$, calcium carbonate; N/A, not applicable; NTU, nephelometric turbidity units; (ch), chronic standard; mL, milliliters; --, no value; LRL, laboratory reporting level; <, less than]

\begin{tabular}{|c|c|c|c|c|c|c|c|c|c|c|c|c|}
\hline \multirow{2}{*}{$\begin{array}{l}\text { Constituent } \\
\text { or property }\end{array}$} & \multirow[b]{2}{*}{ Units } & \multirow{2}{*}{$\begin{array}{c}\text { Period } \\
\text { (water year) }\end{array}$} & \multirow{2}{*}{$\begin{array}{l}\text { Number of } \\
\text { samples }\end{array}$} & \multirow{2}{*}{$\begin{array}{l}\text { Number of } \\
\text { censored } \\
\text { values }\end{array}$} & \multirow[b]{2}{*}{ Median' } & \multicolumn{2}{|c|}{ Maximum } & \multirow{2}{*}{$\begin{array}{c}\text { 85th } \\
\text { percentile }{ }^{1}\end{array}$} & \multicolumn{2}{|c|}{ Standard $^{2}$} & \multirow[b]{2}{*}{ Trend $^{3}$} & \multirow[b]{2}{*}{ Comment } \\
\hline & & & & & & Value & Date & & Value & $\begin{array}{c}\text { Number of } \\
\text { exceedances }\end{array}$ & & \\
\hline \multirow[t]{2}{*}{ Dissolved oxygen } & $\mathrm{mg} / \mathrm{L}$ & $2004-2005$ & 11 & 0 & 9.4 & 10.6 & $01 / 13 / 04$ & 10.1 & 6 & 0 & None & Minimum=7.0 \\
\hline & & 2001-2003 & 18 & 0 & 9.4 & 10.3 & $02 / 26 / 02$ & & & 0 & & 15 th percentile $=7.7$ \\
\hline \multirow[t]{2}{*}{$\mathrm{pH}$} & Standard & 2004-2005 & 11 & 0 & 7.7 & 8.3 & $11 / 04 / 04$ & 8.0 & $6.5-9.0$ & 0 & None & Minimum=7.1 \\
\hline & units & 2001-2003 & 18 & 0 & 7.7 & 8.2 & $08 / 05 / 02$ & & & 0 & & 15 th percentile $=7.4$ \\
\hline \multirow[t]{2}{*}{ Specific conductance } & $\mu \mathrm{S} / \mathrm{cm}$ & 2004-2005 & 11 & 0 & 266 & 348 & $03 / 15 / 04$ & 297 & None & N/A & None & \\
\hline & & 2001-2003 & 18 & 0 & 204 & 365 & $02 / 11 / 03$ & & & N/A & & \\
\hline \multirow[t]{2}{*}{ Temperature } & ${ }^{\circ} \mathrm{C}$ & 2004-2005 & 11 & 0 & 3.4 & 15.8 & $08 / 12 / 04$ & 12 & 20 & 0 & N/A & \\
\hline & & $2001-2003$ & 18 & 0 & 3.4 & 16.3 & $08 / 05 / 02$ & & & 0 & & \\
\hline \multirow[t]{2}{*}{ Hardness (computed) } & $\mathrm{mg} / \mathrm{L}$ as & 2004-2005 & 7 & 0 & 96 & 156 & $03 / 15 / 04$ & 129 & None & N/A & (5) & \\
\hline & $\mathrm{CaCO}_{3}$ & 2001-2003 & 12 & 0 & 60 & 129 & $08 / 05 / 02$ & & & N/A & & \\
\hline \multirow[t]{2}{*}{ Calcium } & $\mathrm{mg} / \mathrm{L}^{3}$ & 2004-2005 & 7 & 0 & 33 & 55 & $03 / 15 / 04$ & 44 & None & N/A & None & \\
\hline & & 2001-2003 & 12 & 0 & 20 & 44 & $08 / 05 / 02$ & & & N/A & & \\
\hline \multirow[t]{2}{*}{ Magnesium } & $\mathrm{mg} / \mathrm{L}$ & $2004-2005$ & 7 & 0 & 3.7 & 4.2 & $03 / 15 / 04$ & 4.7 & None & N/A & None & \\
\hline & & 2001-2003 & 12 & 0 & 3.0 & 4.8 & $08 / 05 / 02$ & & & N/A & & \\
\hline \multirow[t]{2}{*}{ Ammonia } & $\mathrm{mg} / \mathrm{L}$ & 2004-2005 & 11 & 6 & 0 & 0.021 & $03 / 15 / 04$ & 0.007 & None & N/A & (5) & Current LRL $=0.010$ \\
\hline & & 2001-2003 & 18 & 13 & 0 & 0.010 & $04 / 15 / 03$ & & & N/A & & \\
\hline \multirow{2}{*}{$\begin{array}{l}\text { Un-ionized ammonia } \\
\text { (computed) }\end{array}$} & $\mathrm{mg} / \mathrm{L}$ & 2004-2005 & 11 & 6 & 0 & 0.00007 & $03 / 15 / 04$ & 0.00002 & 0.02 & 0 & (5) & \\
\hline & & $2001-2003$ & 18 & 13 & 0 & 0.00011 & $08 / 30 / 01$ & & & 0 & & \\
\hline \multirow{2}{*}{$\begin{array}{l}\text { Ammonia plus organic } \\
\text { nitrogen (total) }\end{array}$} & $\mathrm{mg} / \mathrm{L}$ & 2004-2005 & 11 & 2 & 0.09 & 0.14 & $05 / 26 / 04$ & 0.14 & None & N/A & None & Current LRL $=0.1$ \\
\hline & & 2001-2003 & 18 & 5 & 0.08 & 0.19 & $04 / 15 / 03$ & & & N/A & & \\
\hline \multirow[t]{2}{*}{ Nitrite plus nitrate } & $\mathrm{mg} / \mathrm{L}$ & 2004-2005 & 11 & 2 & 0.04 & 0.20 & 03/15/04 & 0.10 & ${ }^{6} 100$ & 0 & None & Current LRL $=0.016$ \\
\hline & & $2001-2003$ & 18 & 2 & 0.05 & 0.49 & $04 / 15 / 03$ & & & 0 & & \\
\hline \multirow[t]{2}{*}{ Nitrite } & $\mathrm{mg} / \mathrm{L}$ & 2004-2005 & 11 & 5 & 0.001 & 0.001 & ${ }^{4} 04 / 12 / 05$ & 0.001 & 0.05 & 0 & (5) & Current LRL $=0.002$ \\
\hline & & 2001-2003 & 18 & 16 & 0 & 0.002 & $12 / 06 / 01$ & & & 0 & & \\
\hline \multirow[t]{2}{*}{ Orthophosphate } & $\mathrm{mg} / \mathrm{L}$ & $2004-2005$ & 11 & 11 & 0 & ${ }^{7}<0.006$ & - & 0 & None & N/A & (5) & Current LRL $=0.006$ \\
\hline & & $2001-2003$ & 18 & 18 & 0 & ${ }^{7}<0.007$ & - & & & N/A & & \\
\hline \multirow[t]{2}{*}{ Phosphorus (total) } & $\mathrm{mg} / \mathrm{L}$ & 2004-2005 & 11 & 0 & 0.006 & 0.015 & $03 / 15 / 04$ & 0.011 & 0.1 & 0 & None & Current LRL $=0.004$ \\
\hline & & 2001-2003 & 18 & 4 & 0.005 & 0.021 & $05 / 01 / 01$ & & & 0 & & \\
\hline \multirow[t]{2}{*}{ Aluminum } & $\mu \mathrm{g} / \mathrm{L}$ & 2004-2005 & 7 & 0 & 39 & 81 & $06 / 08 / 05$ & 81 & 87 (ch) & 0 & None & Concern \\
\hline & & $2001-2003$ & 12 & 0 & 45 & 240 & $05 / 01 / 01$ & & & 2 & & \\
\hline
\end{tabular}


Table 2. Summary of measured constituents and properties for Coal Creek above Mouth at Crested Butte station 385224106590100.-Continued

[This station name was changed from Coal Creek above Mouth near Crested Butte, CO, to Coal Creek above Mouth at Crested Butte, CO, in 2003. mg/L, milligrams per liter; $\mu$ S/cm, microsiemens per centimeter at $25^{\circ}$ Celsius; $\mu \mathrm{g} / \mathrm{L}$, micrograms per liter; ${ }^{\circ} \mathrm{C}$, degrees Celsius; $\mathrm{CaCO}_{3}$, calcium carbonate; N/A, not applicable; NTU, nephelometric turbidity units; (ch), chronic standard; mL, milliliters; --, no value; $\mathrm{LRL}$, laboratory reporting level; <, less than]

\begin{tabular}{|c|c|c|c|c|c|c|c|c|c|c|c|c|}
\hline \multirow[b]{2}{*}{$\begin{array}{l}\text { Constituent } \\
\text { or property }\end{array}$} & \multirow[b]{2}{*}{ Units } & \multirow{2}{*}{$\begin{array}{c}\text { Period } \\
\text { (water year) }\end{array}$} & \multirow{2}{*}{$\begin{array}{l}\text { Number of } \\
\text { samples }\end{array}$} & \multirow{2}{*}{$\begin{array}{c}\text { Number of } \\
\text { censored } \\
\text { values }\end{array}$} & \multirow[b]{2}{*}{ Median' } & \multicolumn{2}{|c|}{ Maximum } & \multirow{2}{*}{$\begin{array}{c}\text { 85th } \\
\text { percentile }^{1}\end{array}$} & \multicolumn{2}{|c|}{ Standard $^{2}$} & \multirow[b]{2}{*}{ Trend $^{3}$} & \multirow[b]{2}{*}{ Comment } \\
\hline & & & & & & Value & Date & & Value & $\begin{array}{c}\text { Number of } \\
\text { exceedances }\end{array}$ & & \\
\hline \multirow[t]{2}{*}{ Cadmium } & $\mu \mathrm{g} / \mathrm{L}$ & $2004-2005$ & 7 & 0 & 0.76 & 2.4 & $04 / 12 / 05$ & 2.4 & $1.8(\mathrm{ch})$ & 2 & None & High concern \\
\hline & & $2001-2003$ & 12 & 0 & 0.55 & 6.2 & $04 / 15 / 03$ & & & 3 & & \\
\hline \multirow[t]{2}{*}{ Copper } & $\mu \mathrm{g} / \mathrm{L}$ & 2004-2005 & 7 & 0 & 2.7 & 3.4 & $06 / 08 / 05$ & 4.4 & $7.1(\mathrm{ch})$ & 0 & None & Concern \\
\hline & & $2001-2003$ & 12 & 2 & 2.4 & 17.0 & $05 / 01 / 01$ & & & 2 & & \\
\hline \multirow[t]{2}{*}{ Iron } & $\mu \mathrm{g} / \mathrm{L}$ & 2004-2005 & 7 & 2 & 8 & 30 & $05 / 26 / 04$ & 36 & None & N/A & None & \\
\hline & & 2001-2003 & 12 & 0 & 22 & 70 & 05/01/01 & & & N/A & & \\
\hline \multirow[t]{2}{*}{ Lead } & $\mu \mathrm{g} / \mathrm{L}$ & 2004-2005 & 7 & 5 & 0 & 0.5 & ${ }^{4} 06 / 08 / 05$ & 0 & $1.8(\mathrm{ch})$ & 0 & (5) & \\
\hline & & 2001-2003 & 9 & 9 & 0 & ${ }^{7}<1.0$ & -- & & & 0 & & \\
\hline \multirow[t]{2}{*}{ Manganese } & $\mu \mathrm{g} / \mathrm{L}$ & 2004-2005 & 7 & 0 & 25 & 450 & $03 / 15 / 04$ & 277 & 1509 & 0 & None & \\
\hline & & $2001-2003$ & 12 & 0 & 40 & 598 & $04 / 15 / 03$ & & & 0 & & \\
\hline \multirow[t]{2}{*}{ Silver } & $\mu \mathrm{g} / \mathrm{L}$ & 2004-2005 & 7 & 7 & 0 & ${ }^{7}<0.2$ & -- & 0 & $0.20(\mathrm{ch})$ & 0 & (5) & Current LRL $=0.2$ \\
\hline & & $2001-2003$ & 12 & 11 & 0 & 0.1 & $04 / 23 / 02$ & & & 0 & & \\
\hline \multirow[t]{2}{*}{ Zinc } & $\mu \mathrm{g} / \mathrm{L}$ & 2004-2005 & 7 & 0 & 141 & 526 & $04 / 12 / 05$ & 526 & $94(\mathrm{ch})$ & 5 & None & High concern \\
\hline & & $2001-2003$ & 12 & 0 & 121 & 1,120 & $04 / 15 / 03$ & & & 8 & & \\
\hline \multirow[t]{2}{*}{ Suspended sediment } & $\mathrm{mg} / \mathrm{L}$ & 2004-2005 & 7 & 1 & 4 & 13 & $04 / 12 / 05$ & 12 & None & N/A & None & \\
\hline & & $2001-2003$ & 12 & 1 & 2 & 13 & $04 / 15 / 03$ & & & N/A & & \\
\hline \multirow[t]{2}{*}{ Turbidity } & NTU & 2004-2005 & 7 & 0 & 2 & 6 & $03 / 15 / 04$ & 6 & None & N/A & N/A & \\
\hline & & $2001-2003$ & 11 & 0 & 3 & 43 & $05 / 01 / 01$ & & & N/A & & \\
\hline \multirow[t]{2}{*}{ Escherichia coli } & Colonies & 2004-2005 & 11 & 4 & 1 & 22 & $07 / 25 / 05$ & (8) & 126 & 0 & None & Geometric mean $=2.4$ \\
\hline & per $100 \mathrm{~mL}$ & $2001-2003$ & 18 & 4 & 1 & 19 & $08 / 26 / 03$ & & & 0 & & \\
\hline
\end{tabular}

${ }^{1}$ Censored values were replaced with 0 to compute median and 85 th percentiles (coliform censored values replaced with 1 ) see "Definitions of Terms" section.

${ }^{2}$ Colorado Department of Public Health and Environment, classification and numeric standards for Gunnison and Lower Dolores River Basins (2006); and USEPA, Quality criteria for water (1986).

${ }^{3}$ Period of record for trend analysis is water year 2001-water year 2005; flow-adjusted seasonal Kendall method, Helsel and Hirsch, Statistical Methods in Water Resources (1993).

${ }^{4}$ Multiple dates for maximum

${ }^{5}$ Statistic cannot be computed due to number of censored values or insufficient data.

${ }^{6}$ Instream standard for nitrate.

${ }^{7}$ All values censored, current LRL used for maximum.

${ }^{8}$ Use geometric mean for comparison to standard. 


\section{Washington Gulch below Woods Creek at Mount Crested Butte, CO}

Current Reason for Inclusion: To characterize nutrient concentrations. This station is part of the rotational group of stations.

\section{General Station Information:}

Location: 50 feet downstream from the confluence with Woods Creek.

Station Type: USGS water quality

Latitude: 385325

Longitude: 1065812

Drainage area: Not determined

Stream segment: 9
HUC: 14020001

\section{USGS Data Summary:}

Period of Record:

Water quality: November 2000-September 2005

General Chemistry:

Water type: No major-ion data

Hardness: No major-ion data

pH: Low concern

Dissolved oxygen: Low concern

Nutrients:

Total phosphorus: High concern

E. coli: Low concern

Trace Elements/Metals: No data

Other constituents of concern: None

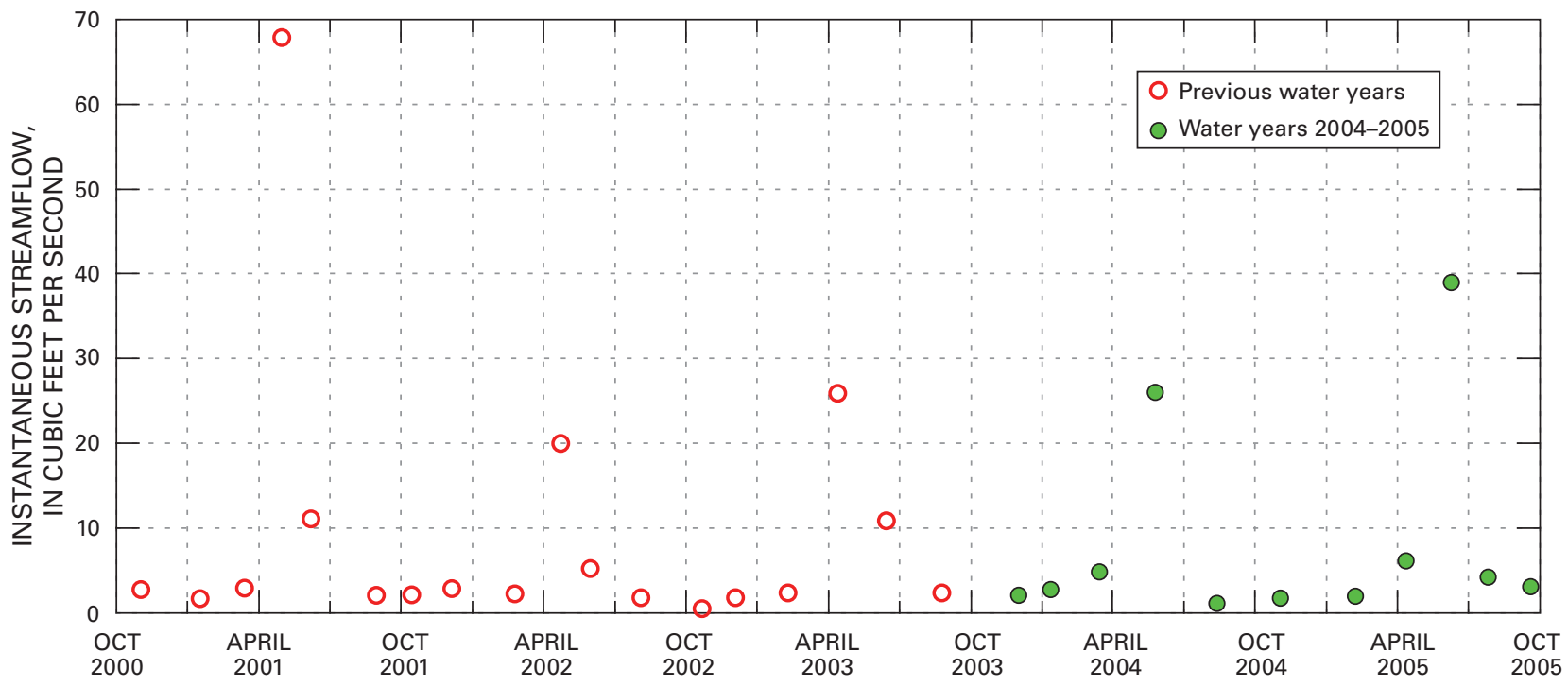

Figure 4. Time distribution and streamflow of water-quality samples for Washington Gulch below Woods Creek at Mount Crested Butte. 

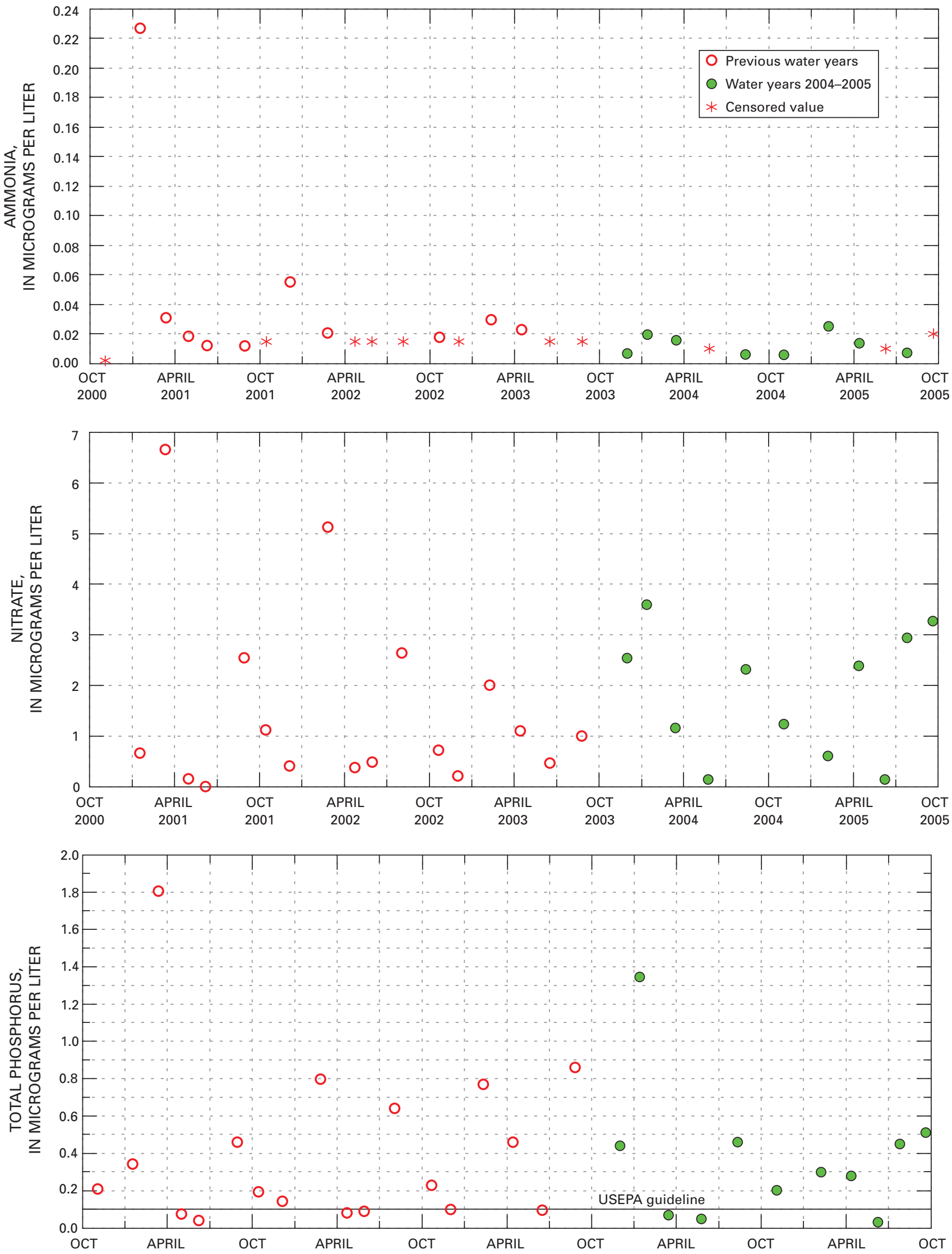

Figure 5. Distribution of selected water-quality constituents relative to time for Washington Gulch below Woods Creek at Mount Crested Butte. 
Table 3. Summary of measured constituents and properties for Washington Gulch below Woods Creek station 385325106581200.

[mg/L, milligrams per liter; $\mu \mathrm{S} / \mathrm{cm}$, microsiemens per centimeter at $25^{\circ}$ Celsius; $\mu \mathrm{g} / \mathrm{L}$, micrograms per liter; ${ }^{\circ} \mathrm{C}$, degrees Celsius; N/A, not applicable; mL, milliliters; --, no value; $\mathrm{LRL}$, laboratory reporting level]

\begin{tabular}{|c|c|c|c|c|c|c|c|c|c|c|c|c|}
\hline \multirow{2}{*}{$\begin{array}{l}\text { Constituent } \\
\text { or property }\end{array}$} & \multirow[b]{2}{*}{ Units } & \multirow{2}{*}{$\begin{array}{c}\text { Period } \\
\text { (water year) }\end{array}$} & \multirow{2}{*}{$\begin{array}{l}\text { Number of } \\
\text { samples }\end{array}$} & \multirow{2}{*}{$\begin{array}{c}\text { Number of } \\
\text { censored } \\
\text { values }\end{array}$} & \multirow[b]{2}{*}{ Median' } & \multicolumn{2}{|c|}{ Maximum } & \multirow{2}{*}{$\begin{array}{c}\text { 85th } \\
\text { percentile }^{1}\end{array}$} & \multicolumn{2}{|c|}{ Standard $^{2}$} & \multirow[b]{2}{*}{ Trend $^{3}$} & \multirow[b]{2}{*}{ Comment } \\
\hline & & & & & & Value & Date & & Value & $\begin{array}{c}\text { Number of } \\
\text { exceedances }\end{array}$ & & \\
\hline \multirow[t]{2}{*}{ Dissolved oxygen } & $\mathrm{mg} / \mathrm{L}$ & 2004-2005 & 11 & 0 & 7.9 & 10.1 & $04 / 12 / 05$ & 9.9 & 6 & 0 & None & Minimum=6.1 \\
\hline & & 2001-2003 & 18 & 0 & 8.7 & 10.2 & 01/17/01 & & & 0 & & 15 th percentile $=7.0$ \\
\hline \multirow[t]{2}{*}{$\mathrm{pH}$} & Standard & 2004-2005 & 11 & 0 & 8.0 & 8.4 & 01/13/04 & 8.2 & $6.5-9.0$ & 0 & None & Minimum $=7.5$ \\
\hline & units & 2001-2003 & 18 & 0 & 8.0 & 8.4 & $11 / 02 / 00$ & & & 0 & & 15 th percentile $=7.8$ \\
\hline \multirow[t]{2}{*}{ Specific conductance } & $\mu \mathrm{S} / \mathrm{cm}$ & 2004-2005 & 11 & 0 & 252 & 331 & $04 / 12 / 05$ & 285 & None & N/A & None & \\
\hline & & 2001-2003 & 18 & 0 & 236 & 361 & 03/13/01 & & & N/A & & \\
\hline \multirow[t]{2}{*}{ Temperature } & ${ }^{\circ} \mathrm{C}$ & 2004-2005 & 11 & 0 & 4.2 & 15.7 & 08/12/04 & 15.5 & 20 & 0 & N/A & \\
\hline & & 2001-2003 & 18 & 0 & 6.4 & 18.0 & 08/05/02 & & & 0 & & \\
\hline \multirow[t]{2}{*}{ Ammonia } & $\mathrm{mg} / \mathrm{L}$ & 2004-2005 & 11 & 3 & 0.007 & 0.025 & 02/07/05 & 0.025 & None & N/A & $\left({ }^{5}\right)$ & Current LRL $=0.010$ \\
\hline & & 2001-2003 & 18 & 8 & 0.012 & 0.227 & 01/17/01 & & & N/A & & \\
\hline \multirow{2}{*}{$\begin{array}{l}\text { Un-ionized ammonia } \\
\text { (computed) }\end{array}$} & $\mathrm{mg} / \mathrm{L}$ & 2004-2005 & 10 & 3 & 0.00010 & 0.00057 & 01/13/04 & 0.00038 & 0.02 & 0 & $\left({ }^{5}\right)$ & \\
\hline & & 2001-2003 & 18 & 8 & 0.00013 & 0.00221 & 01/17/01 & & & 0 & & \\
\hline \multirow{2}{*}{$\begin{array}{l}\text { Ammonia plus organic } \\
\text { nitrogen (total) }\end{array}$} & $\mathrm{mg} / \mathrm{L}$ & 2004-2005 & 11 & 0 & 0.34 & 0.65 & $04 / 12 / 05$ & 0.58 & None & N/A & None & \\
\hline & & 2001-2003 & 18 & 0 & 0.36 & 2.50 & 03/13/01 & & & N/A & & \\
\hline \multirow[t]{2}{*}{ Nitrite plus nitrate } & $\mathrm{mg} / \mathrm{L}$ & 2004-2005 & 11 & 0 & 2.3 & 3.60 & 01/13/04 & 2.9 & ${ }^{6} 10$ & 0 & None & \\
\hline & & 2001-2003 & 18 & 0 & 0.87 & 6.66 & 03/13/01 & & & 0 & & \\
\hline \multirow[t]{2}{*}{ Nitrite } & $\mathrm{mg} / \mathrm{L}$ & 2004-2005 & 11 & 1 & 0.004 & 0.007 & ${ }^{4} 03 / 15 / 04$ & 0.011 & 0.05 & 0 & None & Current LRL $=0.002$ \\
\hline & & 2001-2003 & 18 & 0 & 0.004 & 0.033 & $01 / 17 / 01$ & & & 0 & & \\
\hline \multirow[t]{2}{*}{ Orthophosphate } & $\mathrm{mg} / \mathrm{L}$ & 2004-2005 & 11 & 0 & 0.24 & 1.21 & 01/13/04 & 064 & None & N/A & None & \\
\hline & & 2001-2003 & 18 & 0 & 0.17 & 1.15 & 03/13/01 & & & N/A & & \\
\hline \multirow[t]{2}{*}{ Phosphorus (total) } & $\mathrm{mg} / \mathrm{L}$ & 2004-2005 & 11 & 0 & 0.30 & 1.35 & 01/13/04 & 0.77 & 0.1 & 5 & None & High concern \\
\hline & & 2001-2003 & 18 & 0 & 0.22 & 1.81 & 03/13/01 & & & 13 & & \\
\hline \multirow[t]{2}{*}{ Escherichia coli } & Colonies & 2004-2005 & 11 & 1 & 12 & 170 & $07 / 25 / 05$ & (7) & 126 & 1 & None & Geometric mean $=18$ \\
\hline & per $100 \mathrm{~mL}$ & 2001-2003 & 18 & 3 & 20 & 1,300 & 03/13/01 & & & 4 & & \\
\hline
\end{tabular}

'Censored values were replaced with 0 to compute median and 85th percentiles (coliform censored values replaced with 1). See "Definitions of Terms" section.

${ }^{2}$ Colorado Department of Public Health and Environment, classification and numeric standards for Gunnison and Lower Dolores River Basins (2006); and USEPA, Quality criteria for water (1986).

${ }^{3}$ Period of record for trend analysis is water year 2001-water year 2005; flow-adjusted seasonal Kendall method, Helsel and Hirsch, Statistical Methods in Water Resources (1993).

${ }^{4}$ Multiple dates for maximum.

${ }^{5}$ Statistic cannot be computed due to number of censored values or insufficient data.

${ }^{6}$ Instream standard for nitrate.

${ }^{7}$ Use geometric mean for comparison to standard. 


\section{Slate River near Crested Butte, CO}

Current Reason for Inclusion: Long-term monitoring. This station identifies water quality downstream from Crested Butte.

Historic Reasons for Inclusion: This station is in the segment on the 303(d) list for 1998. Long-term monitoring. The station also characterizes water quality downstream from Crested Butte.

\section{General Station Information:}

Location: 400 feet downstream from Washington Gulch (1 mile east of Crested Butte and 6.3 miles upstream from mouth).

Station Type: USGS water-quality and streamflow gaging

Latitude: 385211

Longitude: 1065808

\section{USGS Data Summary:}

Period of Record:

Water quality:

March 1995-September 2005

Streamflow gaging:

April 1940-September 1951

October 1993-September 2005

General Chemistry:

Water type: Calcium carbonate

Hardness: Moderately hard

pH: Low concern

Dissolved oxygen: Low concern

Nutrients:

Total phosphorus: Concern

E. coli: Low concern

Trace Elements/Metals:

Concern: Zinc

Other constituents of concern: None
Drainage area: $68.9 \mathrm{mi}^{2}$

Stream segment: 7
HUC: 14020002 

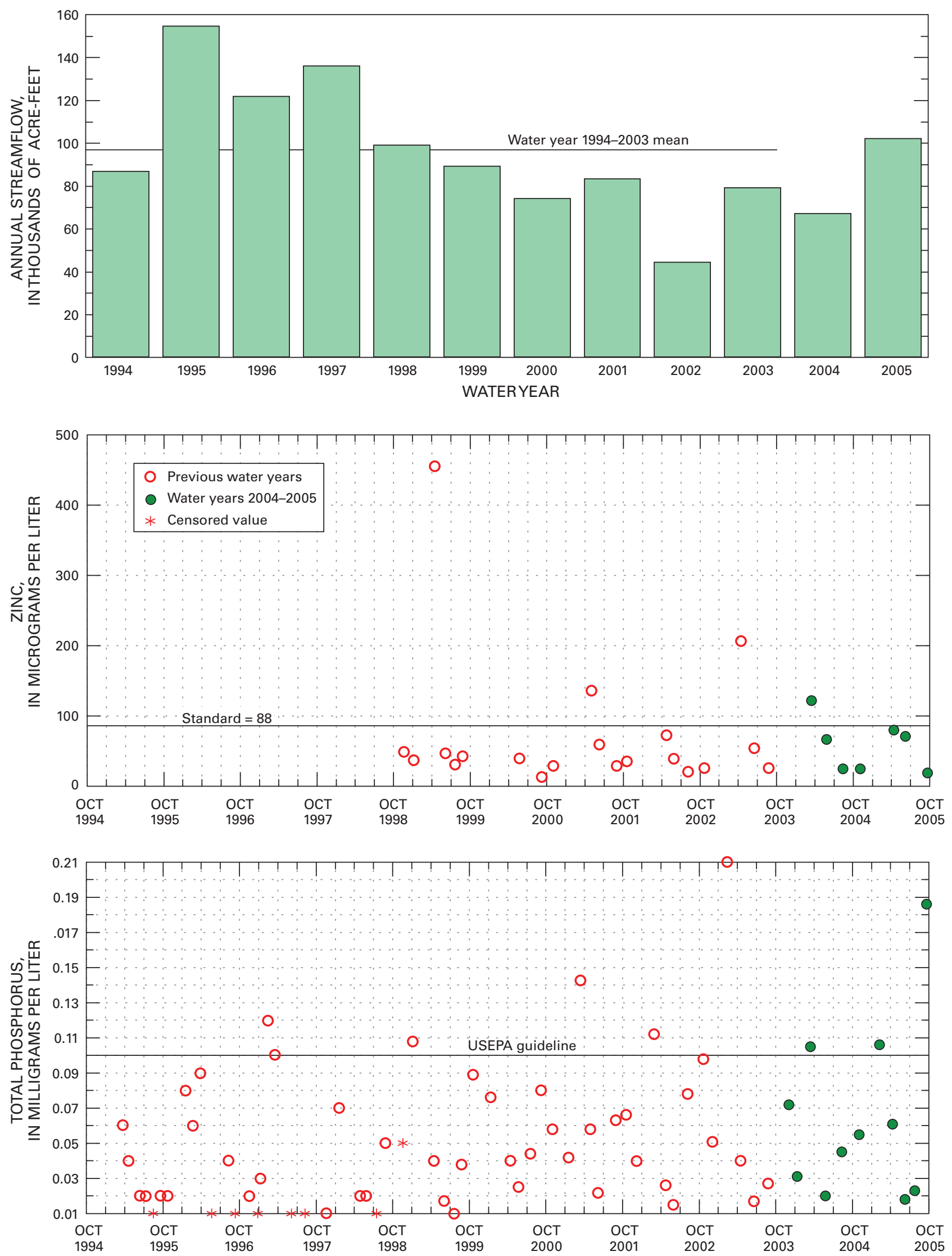

Figure 7. Annual streamflow and distribution of selected water-quality constituents relative to time for Slate River near Crested Butte. 
Table 4. Summary of measured constituents and properties for Slate River near Crested Butte station 09111500.

[mg/L, milligrams per liter; $\mu \mathrm{S} / \mathrm{cm}$, microsiemens per centimeter at $25^{\circ}$ Celsius; $\mu \mathrm{g} / \mathrm{L}$, micrograms per liter; ${ }^{\circ} \mathrm{C}$, degrees Celsius; $\mathrm{CaCO}_{3}$, calcium carbonate; $\mathrm{N} / \mathrm{A}$, not applicable; $\mathrm{NTU}$, nephelometric turbidity units; (ch), chronic standard; $\mathrm{mL}$, milliliters; --, no value; LRL, laboratory reporting level; <, less than; BOD, biochemical oxygen demand]

\begin{tabular}{|c|c|c|c|c|c|c|c|c|c|c|c|c|}
\hline \multirow{2}{*}{$\begin{array}{l}\text { Constituent } \\
\text { or property }\end{array}$} & \multirow[b]{2}{*}{ Units } & \multirow{2}{*}{$\begin{array}{c}\text { Period } \\
\text { (water year) }\end{array}$} & \multirow{2}{*}{$\begin{array}{l}\text { Number of } \\
\text { samples }\end{array}$} & \multirow{2}{*}{$\begin{array}{c}\text { Number of } \\
\text { censored } \\
\text { values }\end{array}$} & \multirow[b]{2}{*}{ Median' } & \multicolumn{2}{|c|}{ Maximum } & \multirow{2}{*}{$\begin{array}{c}\text { 85th } \\
\text { percentile }^{1}\end{array}$} & \multicolumn{2}{|c|}{ Standard ${ }^{2}$} & \multirow[b]{2}{*}{ Trend $^{3}$} & \multirow[b]{2}{*}{ Comment } \\
\hline & & & & & & Value & Date & & Value & $\begin{array}{c}\text { Number of } \\
\text { exceedances }\end{array}$ & & \\
\hline \multirow[t]{2}{*}{ Dissolved oxygen } & $\mathrm{mg} / \mathrm{L}$ & $2004-2005$ & 11 & 0 & 8.8 & 10.2 & $01 / 13 / 04$ & 9.9 & 6 & 0 & Down & Minimum=6.2 \\
\hline & & 1995-2003 & 58 & 0 & 9.2 & 10.6 & $11 / 19 / 96$ & & & 0 & & 15 th percentile $=7.6$ \\
\hline \multirow[t]{2}{*}{$\mathrm{pH}$} & Standard & 2004-2005 & 11 & 0 & 7.4 & 7.8 & ${ }^{4} 11 / 04 / 04$ & 7.8 & $6.5-9.0$ & 0 & None & Minimum $=7.0$ \\
\hline & units & 1995-2003 & 58 & 0 & 7.6 & 8.3 & 05/20/96 & & & 0 & & 15 th percentile $=7.3$ \\
\hline \multirow[t]{2}{*}{ Specific conductance } & $\mu \mathrm{S} / \mathrm{cm}$ & 2004-2005 & 14 & 0 & 174 & 213 & $04 / 12 / 05$ & 206 & None & N/A & None & \\
\hline & & 1995-2003 & 106 & 0 & 158 & 268 & 03/15/94 & & & N/A & & \\
\hline \multirow[t]{2}{*}{ Temperature } & ${ }^{\circ} \mathrm{C}$ & 2004-2005 & 14 & 0 & 7.4 & 18.7 & $07 / 19 / 04$ & 12.0 & 20 & 0 & N/A & \\
\hline & & 1995-2003 & 106 & 0 & 4.0 & 18.0 & 08/05/02 & & & 0 & & \\
\hline \multirow[t]{2}{*}{ Hardness (computed) } & $\mathrm{mg} / \mathrm{L}$ as & 2004-2005 & 7 & 0 & 73 & 90 & $04 / 12 / 05$ & 78 & None & N/A & None & \\
\hline & $\mathrm{CaCO}_{3}$ & $1995-2003$ & 35 & 0 & 66 & 87 & $03 / 25 / 95$ & & & N/A & $(99-05)$ & \\
\hline \multirow[t]{2}{*}{ Calcium } & $\mathrm{mg} / \mathrm{L}$ & 2004-2005 & 7 & 0 & 24 & 29 & $04 / 12 / 05$ & 26 & None & N/A & $\left(5^{5}\right)$ & \\
\hline & & 1995-2003 & 35 & 0 & 22 & 29 & $10 / 17 / 01$ & & & N/A & & \\
\hline \multirow[t]{2}{*}{ Magnesium } & $\mathrm{mg} / \mathrm{L}$ & 2004-2005 & 7 & 0 & 2.9 & 4.7 & $04 / 12 / 05$ & 3.7 & None & N/A & $\left({ }^{5}\right)$ & \\
\hline & & $1995-2003$ & 35 & 0 & 2.7 & 4.2 & $03 / 25 / 95$ & & & N/A & & \\
\hline \multirow[t]{2}{*}{ Ammonia } & $\mathrm{mg} / \mathrm{L}$ & 2004-2005 & 11 & 4 & 0.009 & 0.158 & $03 / 16 / 04$ & 0.181 & None & N/A & None & Current LRL $=0.010$ \\
\hline & & $1995-2003$ & 56 & 7 & 0.060 & 0.779 & $02 / 27 / 02$ & & & N/A & & \\
\hline \multirow{2}{*}{$\begin{array}{l}\text { Un-ionized ammonia } \\
\text { (computed) }\end{array}$} & $\mathrm{mg} / \mathrm{L}$ & 2004-2005 & 11 & 4 & 0.00001 & 0.00046 & $08 / 12 / 05$ & 0.00092 & 0.02 & 0 & $\left({ }^{5}\right)$ & \\
\hline & & 1995-2003 & 56 & 7 & 0.00028 & 0.00370 & $08 / 25 / 99$ & & & 0 & & \\
\hline \multirow{2}{*}{$\begin{array}{l}\text { Ammonia plus organic } \\
\text { nitrogen (total) }\end{array}$} & $\mathrm{mg} / \mathrm{L}$ & 2004-2005 & 11 & 0 & 0.10 & 0.28 & $03 / 16 / 04$ & 0.32 & None & N/A & Down & \\
\hline & & 1995-2003 & 56 & 18 & 0.16 & 0.96 & $02 / 27 / 02$ & & & N/A & & \\
\hline \multirow[t]{2}{*}{ Nitrite plus nitrate } & $\mathrm{mg} / \mathrm{L}$ & 2004-2005 & 11 & 0 & 0.18 & 0.41 & $12 / 03 / 03$ & 0.36 & ${ }^{6} 10$ & 0 & None & \\
\hline & & $1995-2003$ & 56 & 0 & 0.17 & 0.71 & $02 / 18 / 97$ & & & 0 & & \\
\hline \multirow[t]{2}{*}{ Nitrite } & $\mathrm{mg} / \mathrm{L}$ & 2004-2005 & 11 & 1 & 0.002 & 0.007 & $09 / 20 / 05$ & 0.01 & 0.05 & 0 & $\left({ }^{5}\right)$ & Current LRL $=0.002$ \\
\hline & & 1995-2003 & 56 & 25 & 0.002 & 0.030 & ${ }^{4} 10 / 24 / 95$ & & & 0 & & \\
\hline \multirow[t]{2}{*}{ Orthophosphate } & $\mathrm{mg} / \mathrm{L}$ & 2004-2005 & 11 & 1 & 0.019 & 0.145 & $09 / 20 / 05$ & 0.07 & None & N/A & None & Current LRL $=0.006$ \\
\hline & & $1995-2003$ & 56 & 15 & 0.021 & 0.162 & $02 / 11 / 03$ & & & N/A & & \\
\hline \multirow[t]{2}{*}{ Phosphorus (total) } & $\mathrm{mg} / \mathrm{L}$ & 2004-2005 & 11 & 0 & 0.055 & 0.186 & $09 / 20 / 05$ & 0.090 & 0.1 & 3 & up & Concern \\
\hline & & 1995-2003 & 56 & 8 & 0.040 & 0.210 & $02 / 11 / 03$ & & & 6 & & \\
\hline \multirow[t]{2}{*}{ Aluminum } & $\mu \mathrm{g} / \mathrm{L}$ & 2004-2005 & 7 & 0 & 7 & 34 & 06/08/05 & 30 & 87 (ch) & 0 & $\left({ }^{5}\right)$ & \\
\hline & & 1999-2003 & 17 & 2 & 20 & 70 & $05 / 02 / 01$ & & & 0 & & \\
\hline Cadmium & $\mu \mathrm{g} / \mathrm{L}$ & 2004-2005 & 7 & 1 & 0.21 & 0.42 & $05 / 26 / 04$ & 0.42 & $1.7(\mathrm{ch})$ & 0 & $\left({ }^{5}\right)$ & Current LRL $=0.04$ \\
\hline & & 1999-2003 & 20 & 5 & 0.20 & 1.2 & 04/14/99 & & & 0 & & \\
\hline
\end{tabular}


Table 4. Summary of measured constituents and properties for Slate River near Crested Butte station 09111500.—Continued

[mg/L, milligrams per liter; $\mu \mathrm{S} / \mathrm{cm}$, microsiemens per centimeter at $25^{\circ}$ Celsius; $\mu \mathrm{g} / \mathrm{L}$, micrograms per liter; ${ }^{\circ} \mathrm{C}$, degrees Celsius; $\mathrm{CaCO}_{3}$, calcium carbonate; $\mathrm{N} / \mathrm{A}$, not applicable; $\mathrm{NTU}$, nephelometric turbidity units; (ch), chronic standard; $\mathrm{mL}$, milliliters; --, no value; LRL, laboratory reporting level; <, less than; BOD, biochemical oxygen demand]

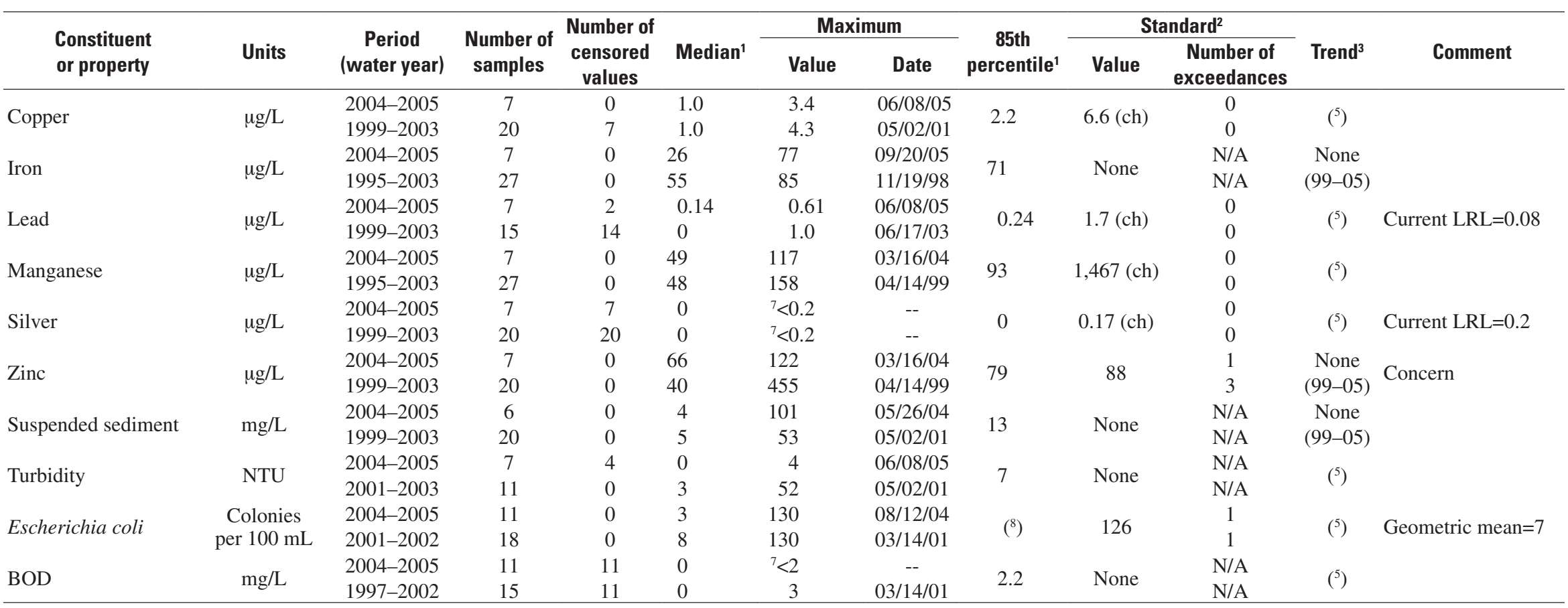

${ }^{1}$ Censored values were replaced with 0 to compute median and 85th percentiles (coliform censored values replaced with 1). See "Definitions of Terms" section.

${ }^{2}$ Colorado Department of Public Health and Environment, classification and numeric standards for Gunnison and Lower Dolores River Basins (2001); and USEPA, Quality criteria for water (1986).

${ }^{3}$ Period of record for trend analysis is water year 1996-water year 2005; flow-adjusted seasonal Kendall method, Helsel and Hirsch, Statistical Methods in Water Resources (1993).

${ }^{4}$ Multiple dates for maximum.

${ }^{5}$ Statistic cannot be computed due to number of censored values or insufficient data.

Instream standard for nitrate.

${ }^{7}$ All values censored, current LRL used for maximum.

${ }^{8}$ Use geometric mean for comparison to standard. 


\section{East River above Slate River near Crested Butte, CO}

Current Reason for Inclusion: This station allows contributions of the Slate and East Rivers to be distinguished and is part of the rotational group of stations.

\section{General Station Information:}

Location: Located 100 feet upstream from confluence with Slate River and 4.7 miles southeast of Crested Butte.

Station Type: USGS water quality

Latitude: 384850

Drainage area: $102 \mathrm{mi}^{2}$

HUC: 14020001

Longitude: 1065336

Stream segment: 5

USGS Data Summary:

Period of Record:

Water quality: April 1995-September 2005

General Chemistry:

Water type: Calcium carbonate

Hardness: Hard

pH: Low concern

Dissolved oxygen: Low concern

Nutrients: Low concern

E. coli: Low concern

Trace Elements/Metals: No data

Other constituents of concern: None

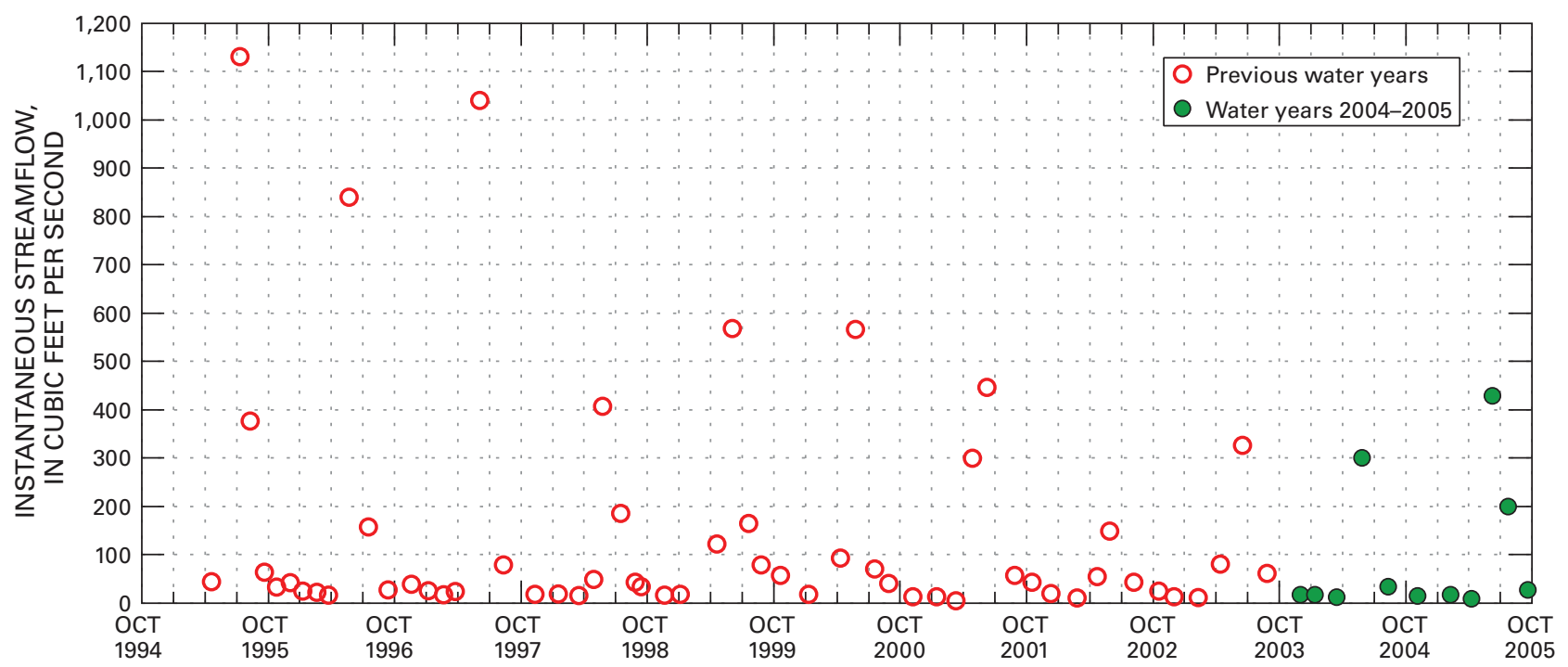

Figure 8. Time distribution and streamflow of water-quality samples for East River above Slate River near Crested Butte. 

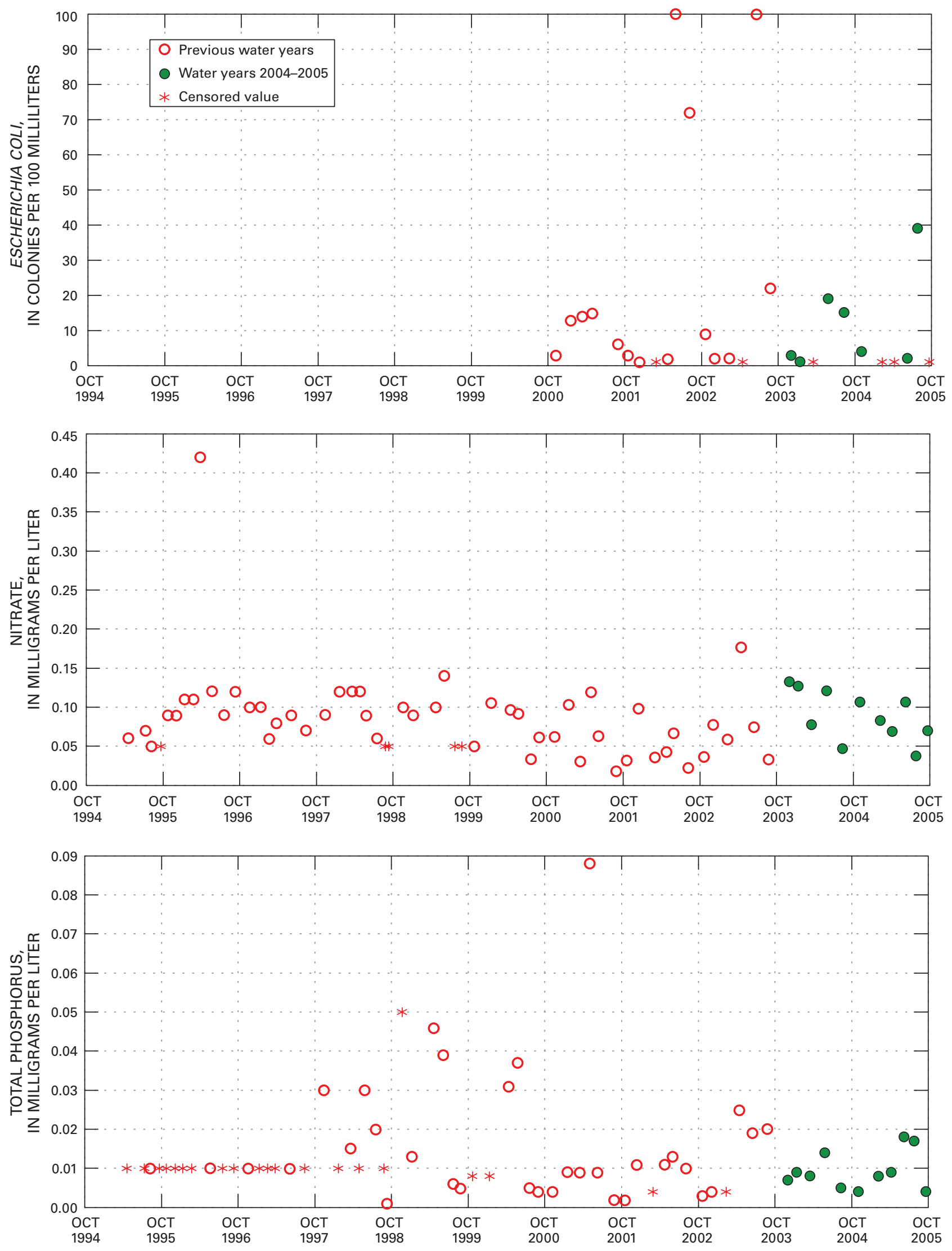

Figure 9. Distribution of selected water-quality constituents relative to time for East River above Slate River near Crested Butte. 
Table 5. Summary of measured constituents and properties for East River above Slate River station 384950106544200.

[mg/L, milligrams per liter; $\mu \mathrm{S} / \mathrm{cm}$, microsiemens per centimeter at $25^{\circ}$ Celsius; $\mu \mathrm{g} / \mathrm{L}$, micrograms per liter; ${ }^{\circ} \mathrm{C}$, degrees Celsius; N/A, not applicable; mL, milliliters; --, no value; LRL, laboratory reporting level; BOD, biochemical oxygen demand]

\begin{tabular}{|c|c|c|c|c|c|c|c|c|c|c|c|c|}
\hline \multirow{2}{*}{$\begin{array}{l}\text { Constituent } \\
\text { or property }\end{array}$} & \multirow[b]{2}{*}{ Units } & \multirow{2}{*}{$\begin{array}{c}\text { Period } \\
\text { (water year) }\end{array}$} & \multirow{2}{*}{$\begin{array}{l}\text { Number of } \\
\text { samples }\end{array}$} & \multirow{2}{*}{$\begin{array}{c}\text { Number of } \\
\text { censored } \\
\text { values }\end{array}$} & \multirow[b]{2}{*}{ Median' } & \multicolumn{2}{|c|}{ Maximum } & \multirow[b]{2}{*}{$\begin{array}{c}\text { 85th } \\
\text { percentile }\end{array}$} & \multicolumn{2}{|c|}{ Standard ${ }^{2}$} & \multirow[b]{2}{*}{ Trend $^{3}$} & \multirow[b]{2}{*}{ Comment } \\
\hline & & & & & & Value & Date & & Value & $\begin{array}{c}\text { Number of } \\
\text { exceedances }\end{array}$ & & \\
\hline \multirow[t]{2}{*}{ Dissolved oxygen } & $\mathrm{mg} / \mathrm{L}$ & 2004-2005 & 11 & 0 & 9.6 & 11.4 & $04 / 07 / 05$ & 10.7 & 6 & 0 & None & Minimum=7.3 \\
\hline & & $1995-2003$ & 56 & 0 & 9.7 & 12.4 & $03 / 26 / 97$ & & & 0 & & 15 th percentile $=7.9$ \\
\hline \multirow[t]{2}{*}{$\mathrm{pH}$} & Standard & 2004-2005 & 11 & 0 & 8.3 & 8.9 & 03/16/04 & 8.4 & $6.5-9.0$ & 0 & None & Minimum=7.4 \\
\hline & units & 1995-2003 & 57 & 0 & 8.2 & 8.6 & ${ }^{4} 03 / 14 / 01$ & & & 0 & & 15 th percentile $=8.1$ \\
\hline \multirow[t]{2}{*}{ Specific conductance } & $\mu \mathrm{S} / \mathrm{cm}$ & 2004-2005 & 11 & 0 & 334 & 356 & 01/13/04 & 337 & None & N/A & None & \\
\hline & & $1995-2003$ & 56 & 0 & 317 & 361 & $11 / 08 / 00$ & & & N/A & & \\
\hline \multirow[t]{2}{*}{ Temperature } & ${ }^{\circ} \mathrm{C}$ & 2004-2005 & 11 & 0 & 3.6 & 16.0 & 08/11/04 & 11.0 & 20 & 0 & N/A & \\
\hline & & $1995-2003$ & 56 & 0 & 4.0 & 14.5 & $05 / 30 / 02$ & & & 0 & & \\
\hline \multirow[t]{2}{*}{ Ammonia } & $\mathrm{mg} / \mathrm{L}$ & 2004-2005 & 11 & 4 & 0.008 & 0.037 & $01 / 13 / 04$ & 0.030 & None & N/A & $\left({ }^{5}\right)$ & Current LRL $=0.01$ \\
\hline & & $1995-2003$ & 56 & 30 & 0 & 0.076 & $12 / 12 / 01$ & & & N/A & & \\
\hline \multirow{2}{*}{$\begin{array}{l}\text { Un-ionized ammonia } \\
\text { (computed) }\end{array}$} & $\mathrm{mg} / \mathrm{L}$ & 2004-2005 & 11 & 4 & 0.0001 & 0.00082 & 03/16/04 & 0.0007 & 0.02 & 0 & $\left({ }^{5}\right)$ & \\
\hline & & $1995-2003$ & 56 & 30 & 0 & 0.00156 & $02 / 19 / 97$ & & & 0 & & \\
\hline \multirow{2}{*}{$\begin{array}{l}\text { Ammonia plus organic } \\
\text { nitrogen (total) }\end{array}$} & $\mathrm{mg} / \mathrm{L}$ & 2004-2005 & 11 & 3 & 0.08 & 0.14 & $01 / 13 / 04$ & 0.13 & None & N/A & $\left({ }^{5}\right)$ & Current LRL=0.1 \\
\hline & & $1995-2003$ & 56 & 29 & 0 & 0.42 & $05 / 02 / 01$ & & & N/A & & \\
\hline \multirow[t]{2}{*}{ Nitrite plus nitrate } & $\mathrm{mg} / \mathrm{L}$ & 2004-2005 & 11 & 0 & 0.08 & 0.13 & $12 / 03 / 03$ & 0.12 & ${ }^{6} 10$ & 0 & None & \\
\hline & & $1995-2003$ & 56 & 5 & 0.08 & 0.42 & $03 / 27 / 96$ & & & 0 & & \\
\hline \multirow[t]{2}{*}{ Nitrite } & $\mathrm{mg} / \mathrm{L}$ & 2004-2005 & 11 & 2 & 0.001 & 0.003 & 03/16/04 & 0.002 & 0.05 & 0 & $\left({ }^{5}\right)$ & Current LRL $=0.002$ \\
\hline & & $1995-2003$ & 56 & 41 & 0 & 0.020 & ${ }^{4} 10 / 25 / 95$ & & & 0 & & \\
\hline \multirow[t]{2}{*}{ Orthophosphate } & $\mathrm{mg} / \mathrm{L}$ & 2004-2005 & 11 & 6 & 0 & 0.004 & ${ }^{4} 04 / 07 / 05$ & 0.004 & None & N/A & $\left({ }^{5}\right)$ & Current LRL $=0.007$ \\
\hline & & $1995-2003$ & 56 & 42 & 0 & 0.040 & 03/27/96 & & & N/A & & \\
\hline \multirow[t]{2}{*}{ Phosphorus (total) } & $\mathrm{mg} / \mathrm{L}$ & 2004-2005 & 11 & 0 & 0.008 & 0.018 & 06/08/05 & 0.020 & 0.1 & 0 & $\left({ }^{5}\right)$ & \\
\hline & & 1995-2003 & 55 & 21 & 0.004 & 0.088 & 05/02/01 & & & 0 & & \\
\hline \multirow[t]{2}{*}{ Escherichia coli } & Colonies per & 2004-2005 & 11 & 4 & 2 & 39 & $07 / 26 / 05$ & $\left({ }^{7}\right)$ & 126 & 0 & $\left({ }^{5}\right)$ & Geometric mean $=5$ \\
\hline & $100 \mathrm{~mL}$ & 2001-2003 & 17 & 2 & 6 & 100 & ${ }^{4} 05 / 30 / 02$ & & & 0 & & \\
\hline \multirow[t]{2}{*}{ BOD } & $\mathrm{mg} / \mathrm{L}$ & 2004-2005 & 11 & 10 & 0 & 1.0 & 09/20/05 & 0.9 & None & N/A & $\left({ }^{5}\right)$ & \\
\hline & & 1995-2003 & 39 & 31 & 0 & 2.6 & ${ }^{4} 04 / 19 / 95$ & & & N/A & & \\
\hline
\end{tabular}

${ }^{1}$ Censored values were replaced with 0 to compute median and 85th percentiles (coliform censored values replaced with 1). See "Definitions of Terms" section.

${ }^{2}$ Colorado Department of Public Health and Environment, classification and numeric standards for Gunnison and Lower Dolores River Basins (2001); and USEPA, Quality criteria for water (1986).

${ }^{3}$ Period of record for trend analysis is water year 1996-water year 2003; flow-adjusted seasonal Kendall method, Helsel and Hirsch, Statistical Methods in Water Resources (1993).

${ }^{4}$ Multiple dates for maximum.

${ }^{5}$ Statistic cannot be computed due to number of censored values or insufficient data.

${ }^{6}$ Instream standard for nitrate.

${ }^{7}$ Use geometric mean for comparison to standard. 


\section{East River below Cement Creek, CO}

Current Reason for Inclusion: Long-term monitoring. First station on East River downstream from the confluence with the Slate River.

\section{General Site Information:}

Location: 1.6 miles downstream from Cement Creek (8.5 miles southeast of Crested Butte).

Station Type: USGS water quality and streamflow gaging

Latitude: 384703

Longitude: 1065613
Drainage area: $238 \mathrm{mi}^{2}$

Stream segment: 5
HUC: 14020001

\section{USGS Data Summary:}

Period of Record:

Water quality:

October 1993-September 2005

Streamflow gaging:

October 1963-September 1972

October 1979-September 1981

October 1993-September 2005

General Chemistry:

Water type: Calcium carbonate

Hardness: Moderately hard

pH: Low concern

Dissolved oxygen: Low concern

Nutrients: Low concern

E. coli: Low concern

Trace Elements/Metals: Low concern (based on historical information 1994-2001)

Other constituents of concern: None

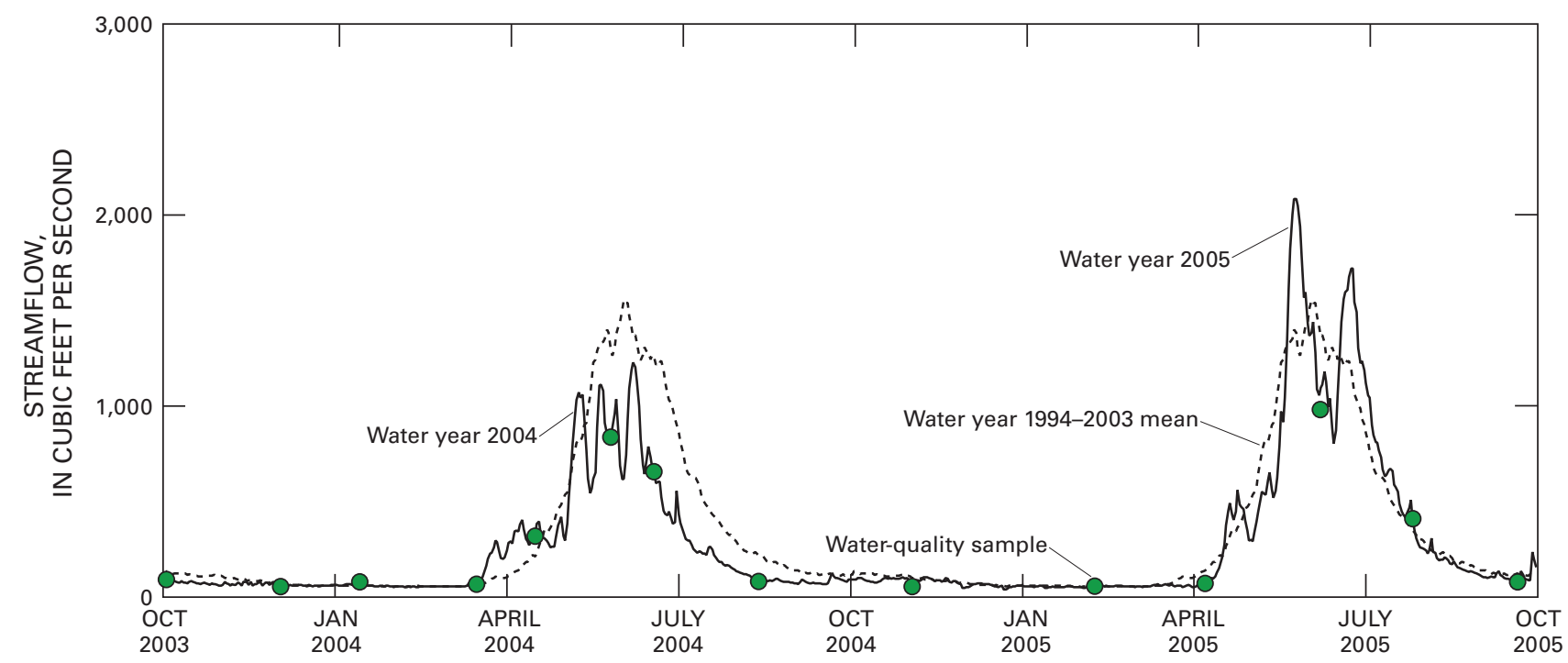

Figure 10. Daily mean streamflow and time distribution of water-quality samples for East River below Cement Creek. 

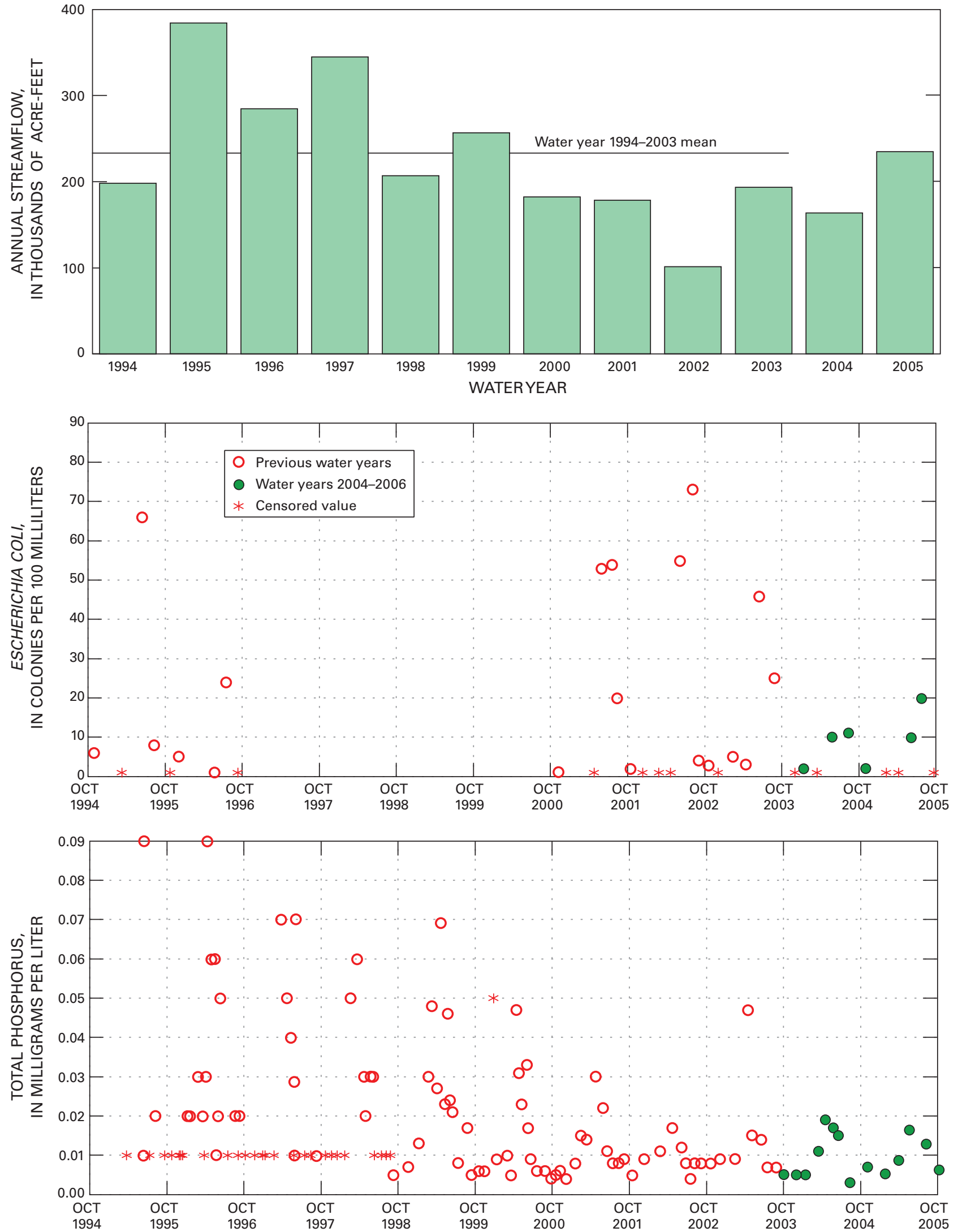

Figure 11. Annual streamflow and distribution of selected water-quality constituents relative to time for East River below Cement Creek. 
[mg/L, milligrams per liter; $\mu \mathrm{S} / \mathrm{cm}$, microsiemens per centimeter at $25^{\circ}$ Celsius; $\mu \mathrm{g} / \mathrm{L}$, micrograms per liter; ${ }^{\circ} \mathrm{C}$, degrees Celsius; N/A, not applicable; $\mathrm{mL}$, milliliters; $\mathrm{LRL}$, laboratory reporting level]

\begin{tabular}{|c|c|c|c|c|c|c|c|c|c|c|c|c|}
\hline \multirow{2}{*}{$\begin{array}{l}\text { Constituent } \\
\text { or property }\end{array}$} & \multirow[b]{2}{*}{ Units } & \multirow{2}{*}{$\begin{array}{c}\text { Period } \\
\text { (water year) }\end{array}$} & \multirow{2}{*}{$\begin{array}{l}\text { Number of } \\
\text { samples }\end{array}$} & \multirow{2}{*}{$\begin{array}{c}\text { Number of } \\
\text { censored } \\
\text { values }\end{array}$} & \multirow[b]{2}{*}{ Median' } & \multicolumn{2}{|c|}{ Maximum } & \multirow{2}{*}{$\begin{array}{c}\text { 85th } \\
\text { percentile }^{1}\end{array}$} & \multicolumn{2}{|c|}{ Standard $^{2}$} & \multirow[b]{2}{*}{ Trend $^{3}$} & \multirow[b]{2}{*}{ Comment } \\
\hline & & & & & & Value & Date & & Value & $\begin{array}{c}\text { Number of } \\
\text { exceedances }\end{array}$ & & \\
\hline \multirow[t]{2}{*}{ Dissolved oxygen } & $\mathrm{mg} / \mathrm{L}$ & 2004-2005 & 14 & 0 & 8.5 & 12.0 & $02 / 08 / 05$ & 10.8 & 6 & 0 & None & Minimum=7.1 \\
\hline & & 1994-2003 & 120 & 0 & 9.4 & 12.7 & $03 / 25 / 94$ & & & 0 & & 15 th percentile $=8.0$ \\
\hline \multirow[t]{2}{*}{$\mathrm{pH}$} & Standard & 2004-2005 & 14 & 0 & 8.4 & 8.8 & ${ }^{4} 01 / 14 / 04$ & 8.6 & $6.5-9.0$ & 0 & None & Minimum=7.6 \\
\hline & units & 1994-2003 & 120 & 0 & 8.3 & 9.0 & ${ }^{4} 02 / 27 / 96$ & & & 0 & & 15 th percentile $=8.0$ \\
\hline \multirow[t]{2}{*}{ Specific conductance } & $\mu \mathrm{S} / \mathrm{cm}$ & 2004-2005 & 14 & 0 & 290 & 328 & 08/13/04 & 314 & None & N/A & None & \\
\hline & & 1994-2003 & 120 & 0 & 278 & 335 & 09/03/02 & & & N/A & & \\
\hline \multirow[t]{2}{*}{ Temperature } & ${ }^{\circ} \mathrm{C}$ & 2004-2005 & 14 & 0 & 7.6 & 15.6 & $07 / 26 / 05$ & 11.9 & 20 & 0 & N/A & \\
\hline & & 1994-2003 & 120 & 0 & 5.6 & 17.2 & 08/05/98 & & & 0 & & \\
\hline \multirow[t]{2}{*}{ Alkalinity } & $\mathrm{mg} / \mathrm{L}$ & 2004-2005 & 14 & 0 & 114 & 142 & ${ }^{4} 01 / 14 / 04$ & 122 & None & N/A & None & \\
\hline & & 1995-2003 & 97 & 0 & 103 & 136 & $02 / 26 / 02$ & & & N/A & & \\
\hline \multirow[t]{2}{*}{ Sulfate } & $\mathrm{mg} / \mathrm{L}$ & 2004-2005 & 14 & 0 & 32 & 40 & $01 / 14 / 04$ & 36 & 250 & 0 & None & \\
\hline & & $1995-2003$ & 112 & 0 & 30 & 40 & $02 / 12 / 03$ & & & 0 & & \\
\hline \multirow[t]{2}{*}{ Chloride } & $\mathrm{mg} / \mathrm{L}$ & 2004-2005 & 14 & 0 & 2.0 & 3.8 & $03 / 16 / 04$ & 2.1 & 250 & 0 & Up & \\
\hline & & $1995-2003$ & 112 & 0 & 1.3 & 3.7 & $01 / 18 / 96$ & & & 0 & & \\
\hline \multirow[t]{2}{*}{ Ammonia } & $\mathrm{mg} / \mathrm{L}$ & 2004-2005 & 14 & 7 & 0.002 & 0.011 & ${ }^{4} 06 / 07 / 05$ & 0.027 & None & N/A & $\left({ }^{5}\right)$ & Current LRL $=0.01$ \\
\hline & & 1994-2003 & 119 & 56 & 0.003 & 0.110 & 03/19/98 & & & N/A & & \\
\hline \multirow{2}{*}{$\begin{array}{l}\text { Un-ionized ammonia } \\
\text { (computed) }\end{array}$} & $\mathrm{mg} / \mathrm{L}$ & 2004-2005 & 14 & 7 & 0.00003 & 0.00074 & $02 / 08 / 05$ & 0.00066 & 0.02 & 0 & $\left({ }^{5}\right)$ & \\
\hline & & 1994-2003 & 119 & 56 & 0.00007 & 0.00705 & 03/19/98 & & & 0 & & \\
\hline \multirow{2}{*}{$\begin{array}{l}\text { Ammonia plus organic } \\
\text { nitrogen (total) }\end{array}$} & $\mathrm{mg} / \mathrm{L}$ & 2004-2005 & 14 & 2 & 0.09 & 0.17 & $04 / 16 / 04$ & 0.19 & None & N/A & $\left({ }^{5}\right)$ & Current LRL $=0.1$ \\
\hline & & $1995-2003$ & 113 & 44 & 0.08 & 0.40 & ${ }^{4} 04 / 23 / 97$ & & & N/A & & \\
\hline \multirow[t]{2}{*}{ Nitrite plus nitrate } & $\mathrm{mg} / \mathrm{L}$ & 2004-2005 & 14 & 0 & 0.08 & 0.19 & $04 / 16 / 04$ & 0.16 & ${ }^{6} 10$ & 0 & None & \\
\hline & & 1994-2003 & 119 & 5 & 0.09 & 0.32 & 05/06/03 & & & 0 & & \\
\hline \multirow[t]{2}{*}{ Nitrite } & $\mathrm{mg} / \mathrm{L}$ & 2004-2005 & 14 & 4 & 0.001 & 0.002 & ${ }^{4} 04 / 07 / 05$ & 0.002 & 0.05 & 0 & $\left({ }^{5}\right)$ & Current LRL $=0.002$ \\
\hline & & 1994-2003 & 119 & 72 & 0 & 0.020 & ${ }^{4} 03 / 27 / 96$ & & & 0 & & \\
\hline \multirow[t]{2}{*}{ Orthophosphate } & $\mathrm{mg} / \mathrm{L}$ & 2004-2005 & 14 & 13 & 0 & 0.003 & $11 / 03 / 04$ & 0.010 & None & N/A & $\left({ }^{5}\right)$ & Current LRL $=0.006$ \\
\hline & & 1994-2003 & 119 & 81 & 0 & 0.040 & $02 / 19 / 98$ & & & N/A & & \\
\hline \multirow[t]{2}{*}{ Phosphorus (total) } & $\mathrm{mg} / \mathrm{L}$ & 2004-2005 & 14 & 0 & 0.008 & 0.019 & $04 / 16 / 04$ & 0.030 & 0.1 & 0 & $\left({ }^{5}\right)$ & \\
\hline & & $1995-2003$ & 113 & 27 & 0.009 & 0.090 & ${ }^{4} 06 / 16 / 95$ & & & 0 & & \\
\hline \multirow[t]{2}{*}{ Suspended sediment } & $\mathrm{mg} / \mathrm{L}$ & 2004-2005 & 13 & 2 & 2 & 10 & 06/07/05 & 19 & None & N/A & None & \\
\hline & & 1995-2003 & 92 & 1 & 3 & 241 & $06 / 16 / 95$ & & & N/A & & \\
\hline Escherichia coli & Colonies per & 2004-2005 & 11 & 5 & 2 & 20 & $07 / 26 / 05$ & $\left({ }^{7}\right)$ & 126 & 0 & $\left({ }^{5}\right)$ & Geometric mean $=5$ \\
\hline & $100 \mathrm{~mL}$ & 1994-2003 & 31 & 8 & 4 & 82 & 06/01/94 & & & 0 & & \\
\hline
\end{tabular}

${ }^{1}$ Censored values were replaced with 0 to compute median and 85th percentiles (coliform censored values replaced with 1). See "Definitions of Terms" section.

${ }^{2}$ Colorado Department of Public Health and Environment, classification and numeric standards for Gunnison and Lower Dolores River Basins (2006); and USEPA, Quality criteria for water (1986).

${ }^{3}$ Period of record for trend analysis is water year 1996-water year 2003; flow-adjusted seasonal Kendall method, Helsel and Hirsch, Statistical Methods in Water Resources (1993).

${ }^{4}$ Multiple dates for maximum.

${ }^{5}$ Statistic cannot be computed due to number of censored values or insufficient data.

${ }^{6}$ Instream standard for nitrate.

${ }^{7}$ Use geometric mean for comparison to standard. 


\section{East River at Almont, CO}

Current Reason for Inclusion: Allows contributions of the East River to be identified. Long-term monitoring.

\section{General Station Information:}

Location: 400 feet upstream from confluence with Taylor River.

Station Type: USGS water quality and streamflow gaging

Latitude: 383952

Longitude: 1065051
Drainage area: $289 \mathrm{mi}^{2}$

Stream segment: 5

HUC: 14020001

\section{USGS Data Summary:}

Period of Record:

Water quality: October 1990-September 2005

Streamflow gaging: October 1934-September 2005

General Chemistry:

Water type: Calcium carbonate

Hardness: Hard

pH: Low concern

Dissolved oxygen: Low concern

Nutrients: Low concern

E. coli: Low concern

Trace Elements/Metals: No data

Other constituents of concern: None

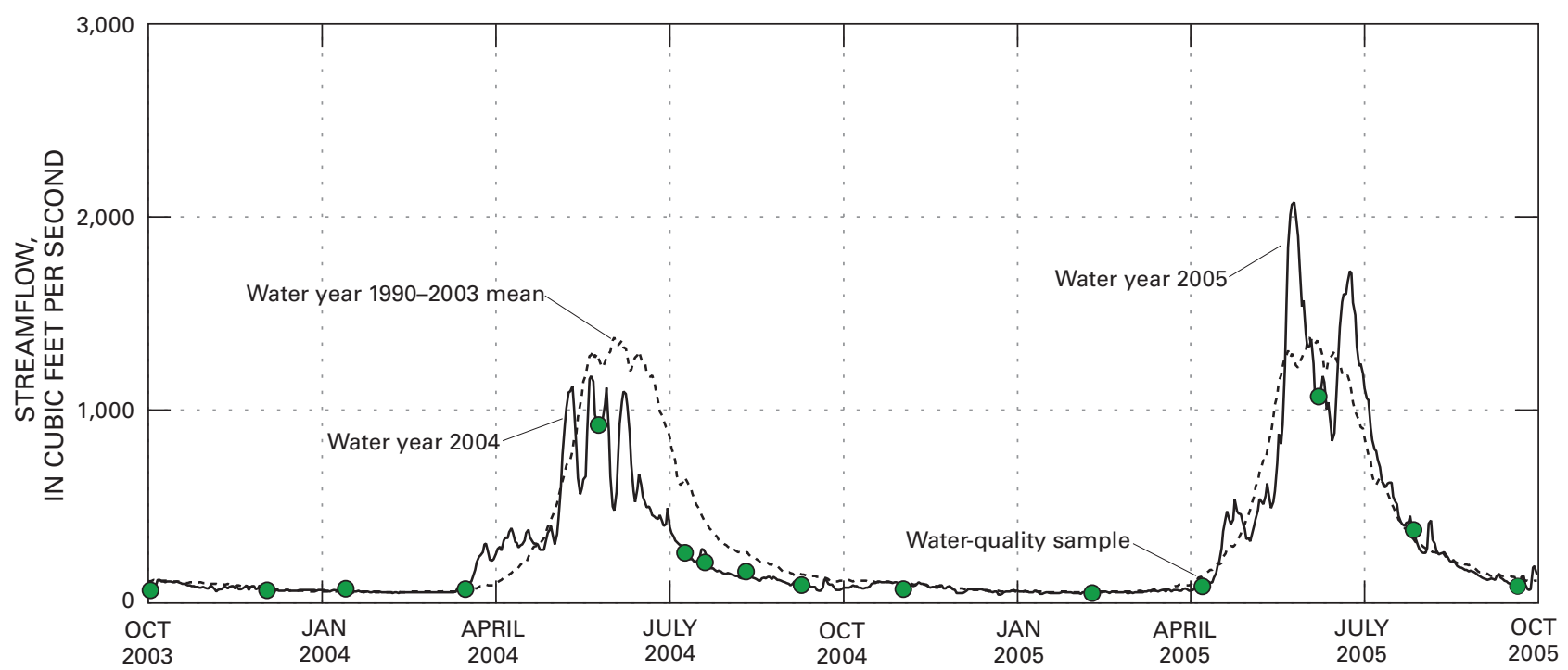

Figure 12. Daily mean streamflow and time distribution of water-quality samples for East River at Almont. 

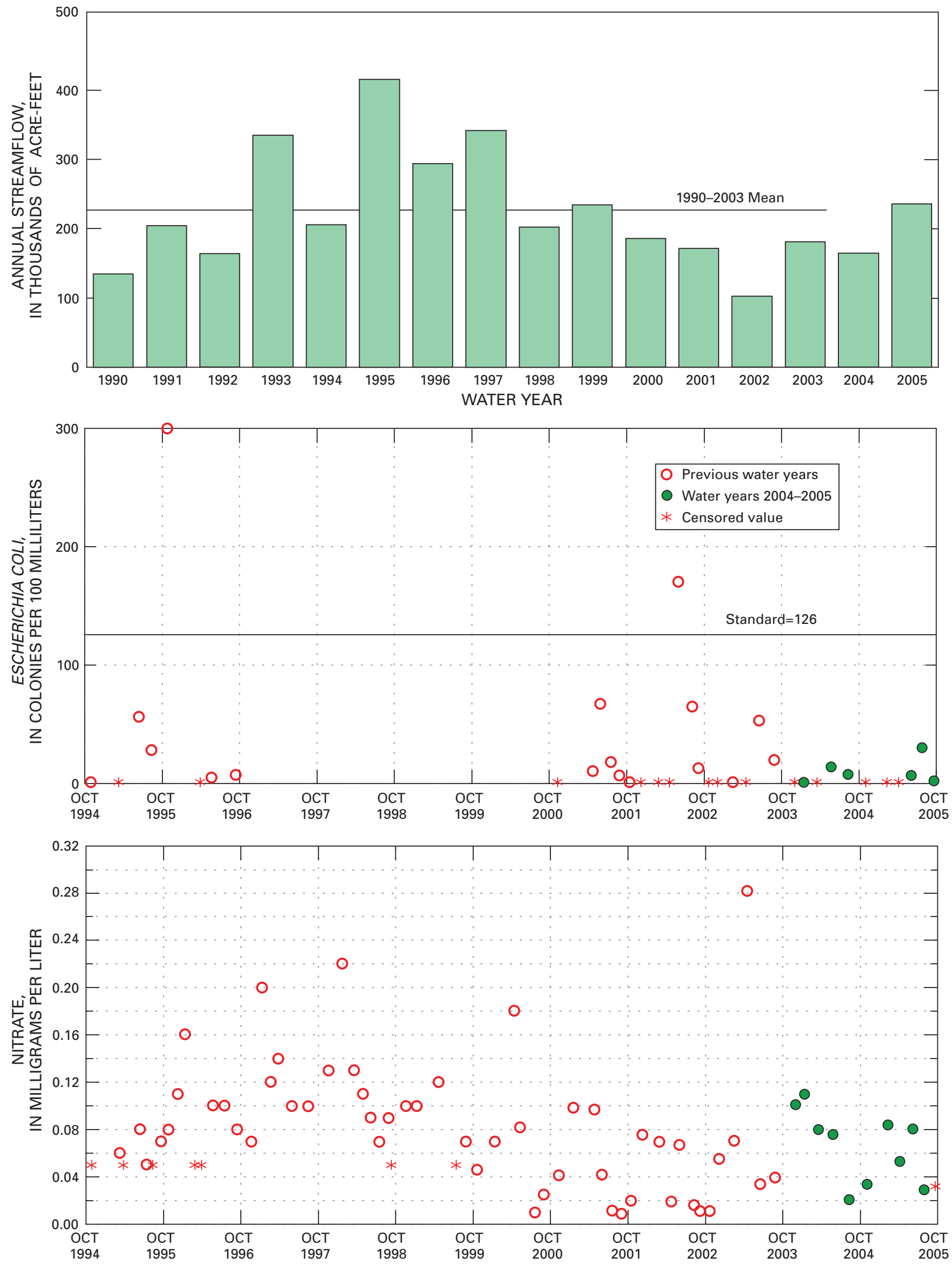

Figure 13. Annual streamflow and distribution of selected water-quality constituents relative to time for East River at Almont. 
Table 7. Summary of measured constituents and properties for East River at Almont station 09112500.

[mg/L, milligrams per liter; $\mu \mathrm{S} / \mathrm{cm}$, microsiemens per centimeter at $25^{\circ}$ Celsius; $\mu \mathrm{g} / \mathrm{L}$, micrograms per liter; ${ }^{\circ} \mathrm{C}$, degrees Celsius; $\mathrm{N} / \mathrm{A}$, not applicable; $\mathrm{mL}$, milliliters; $\mathrm{LRL}$, laboratory reporting level]

\begin{tabular}{|c|c|c|c|c|c|c|c|c|c|c|c|c|}
\hline \multirow[b]{2}{*}{$\begin{array}{l}\text { Constituent } \\
\text { or property }\end{array}$} & \multirow[b]{2}{*}{ Units } & \multirow{2}{*}{$\begin{array}{c}\text { Period } \\
\text { (water year) }\end{array}$} & \multirow[b]{2}{*}{$\begin{array}{l}\text { Number of } \\
\text { samples }\end{array}$} & \multirow{2}{*}{$\begin{array}{c}\text { Number of } \\
\text { censored } \\
\text { values }\end{array}$} & \multirow{2}{*}{ Median' } & \multicolumn{2}{|c|}{ Maximum } & \multirow[b]{2}{*}{$\begin{array}{c}\text { 85th } \\
\text { percentile }^{1}\end{array}$} & \multicolumn{2}{|c|}{ Standard $^{2}$} & \multirow[b]{2}{*}{ Trend $^{3}$} & \multirow[b]{2}{*}{ Comment } \\
\hline & & & & & & Value & Date & & Value & $\begin{array}{c}\text { Number of } \\
\text { exceedances }\end{array}$ & & \\
\hline \multirow[t]{2}{*}{ Dissolved oxygen } & $\mathrm{mg} / \mathrm{L}$ & $2004-2005$ & 11 & 0 & 8.3 & 10.5 & $12 / 03 / 03$ & 11.0 & 6 & 0 & Down & Minimum=7.0 \\
\hline & & $1991-2003$ & 75 & 0 & 9.4 & 12.8 & ${ }^{4} 11 / 17 / 93$ & & & 0 & & 15 th percentile $=8.0$ \\
\hline \multirow[t]{2}{*}{$\mathrm{pH}$} & Standard & 2004-2005 & 11 & 0 & 8.3 & 8.7 & 03/16/04 & 8.6 & $6.5-9.0$ & 0 & None & Minimum $=7.5$ \\
\hline & units & $1991-2003$ & 76 & 0 & 8.3 & 8.9 & ${ }^{4} 11 / 17 / 93$ & & & 0 & & 15 th percentile $=8.1$ \\
\hline \multirow[t]{2}{*}{ Specific conductance } & $\mu \mathrm{S} / \mathrm{cm}$ & 2004-2005 & 15 & 0 & 309 & 349 & 09/09/04 & 330 & None & $\mathrm{N} / \mathrm{A}$ & $\mathrm{Up}$ & \\
\hline & & 1990-2003 & 172 & 0 & 298 & 369 & $10 / 22 / 91$ & & & N/A & & \\
\hline \multirow[t]{2}{*}{ Temperature } & ${ }^{\circ} \mathrm{C}$ & 2004-2005 & 15 & 0 & 11.4 & 16.8 & 07/09/04 & 14 & 20 & 0 & N/A & \\
\hline & & $1990-2003$ & 174 & 0 & 7.4 & 18.5 & $07 / 17 / 02$ & & & 0 & & \\
\hline \multirow[t]{2}{*}{ Ammonia } & $\mathrm{mg} / \mathrm{L}$ & 2004-2005 & 11 & 4 & 0.005 & 0.009 & 06/07/05 & 0.020 & None & N/A & $\left({ }^{5}\right)$ & Current LRL $=0.01$ \\
\hline & & $1994-2003$ & 64 & 36 & 0 & 0.040 & ${ }^{4} 11 / 20 / 96$ & & & N/A & & \\
\hline \multirow{2}{*}{$\begin{array}{l}\text { Un-ionized ammonia } \\
\text { (computed) }\end{array}$} & $\mathrm{mg} / \mathrm{L}$ & 2004-2005 & 11 & 4 & 0.0001 & 0.0005 & 08/11/04 & 0.0007 & 0.02 & 0 & $\left({ }^{5}\right)$ & \\
\hline & & 1994-2003 & 64 & 36 & 0 & 0.0033 & $07 / 17 / 96$ & & & 0 & & \\
\hline \multirow{2}{*}{$\begin{array}{l}\text { Ammonia plus organic } \\
\text { nitrogen (total) }\end{array}$} & $\mathrm{mg} / \mathrm{L}$ & 2004-2005 & 11 & 1 & 0.11 & 0.12 & ${ }^{4} 03 / 16 / 04$ & 0.16 & None & N/A & $\left({ }^{5}\right)$ & Current LRL $=0.1$ \\
\hline & & 1994-2003 & 58 & 25 & 0.07 & 0.36 & $04 / 21 / 99$ & & & N/A & & \\
\hline \multirow[t]{2}{*}{ Nitrite plus nitrate } & $\mathrm{mg} / \mathrm{L}$ & 2004-2005 & 11 & 1 & 0.08 & 0.11 & $01 / 14 / 04$ & 0.11 & ${ }^{6} 10$ & 0 & Down & Current LRL $=0.032$ \\
\hline & & 1994-2003 & 63 & 8 & 0.07 & 0.28 & $04 / 16 / 03$ & & & 0 & & \\
\hline \multirow[t]{2}{*}{ Nitrite } & $\mathrm{mg} / \mathrm{L}$ & 2004-2005 & 11 & 1 & 0.001 & 0.002 & ${ }^{4} 04 / 07 / 05$ & 0.002 & 0.05 & 0 & $\left({ }^{5}\right)$ & Current LRL $=0.002$ \\
\hline & & 1994-2003 & 64 & 44 & 0 & 0.030 & $11 / 20 / 96$ & & & 0 & & \\
\hline \multirow[t]{2}{*}{ Orthophosphate } & $\mathrm{mg} / \mathrm{L}$ & 2004-2005 & 11 & 9 & 0 & 0.003 & ${ }^{4} 11 / 02 / 04$ & 0.001 & None & N/A & $\left({ }^{5}\right)$ & Current LRL $=0.006$ \\
\hline & & 1994-2003 & 63 & 52 & 0 & 0.020 & 06/03/98 & & & N/A & & \\
\hline \multirow[t]{2}{*}{ Phosphorus (total) } & $\mathrm{mg} / \mathrm{L}$ & 2004-2005 & 11 & 0 & 0.009 & 0.023 & $06 / 07 / 05$ & 0.021 & 0.1 & 0 & $\left({ }^{5}\right)$ & \\
\hline & & 1994-2003 & 58 & 17 & 0.005 & 0.064 & $04 / 21 / 99$ & & & 0 & & \\
\hline \multirow[t]{2}{*}{ Escherichia coli } & Colonies & 2004-2005 & 11 & 5 & 1 & 30 & $07 / 27 / 05$ & $\left({ }^{7}\right)$ & 126 & 0 & $\left({ }^{5}\right)$ & Geometric mean $=6$ \\
\hline & per $100 \mathrm{~mL}$ & $1991-2003$ & 42 & 13 & 6 & 300 & $10 / 26 / 95$ & & & 2 & & \\
\hline
\end{tabular}

'Censored values were replaced with 0 to compute median and 85 th percentiles (coliform censored values replaced with 1). See "Definitions of Terms" section.

${ }^{2}$ Colorado Department of Public Health and Environment, classification and numeric standards for Gunnison and Lower Dolores River Basins (2001); and USEPA, Quality criteria for water (1986).

${ }^{3}$ Period of record for trend analysis is water year 1996-water year 2003; flow-adjusted seasonal Kendall method, Helsel and Hirsch, Statistical Methods in Water Resources (1993).

${ }^{4}$ Multiple dates for maximum.

${ }^{5}$ Statistic cannot be computed due to number of censored values or insufficient data.

${ }^{6}$ Instream standard for nitrate.

${ }^{7}$ Use geometric mean for comparison to standard. 


\section{Ohio Creek near Baldwin}

Current Reason for Inclusion: To provide baseline water-quality data in anticipation of possible energy development upstream.

\section{General Station Information:}

Station Type: USGS water quality

Latitude: 384208

Longitude: 1065952
Drainage area: $121 \mathrm{mi}^{2}$

Stream segment: 16

HUC: 14020002

\section{USGS Data Summary:}

Period of Record:

Water quality: December 2003 to September 2005

General Chemistry:

Water type: Insufficient major-ion data

pH: Low concern

Dissolved oxygen: Low concern

Nutrients:

Total phosphorus: Concern

E. coli: Low concern

Trace Elements/Metals:

Iron: Low concern

Other constituents of concern: None

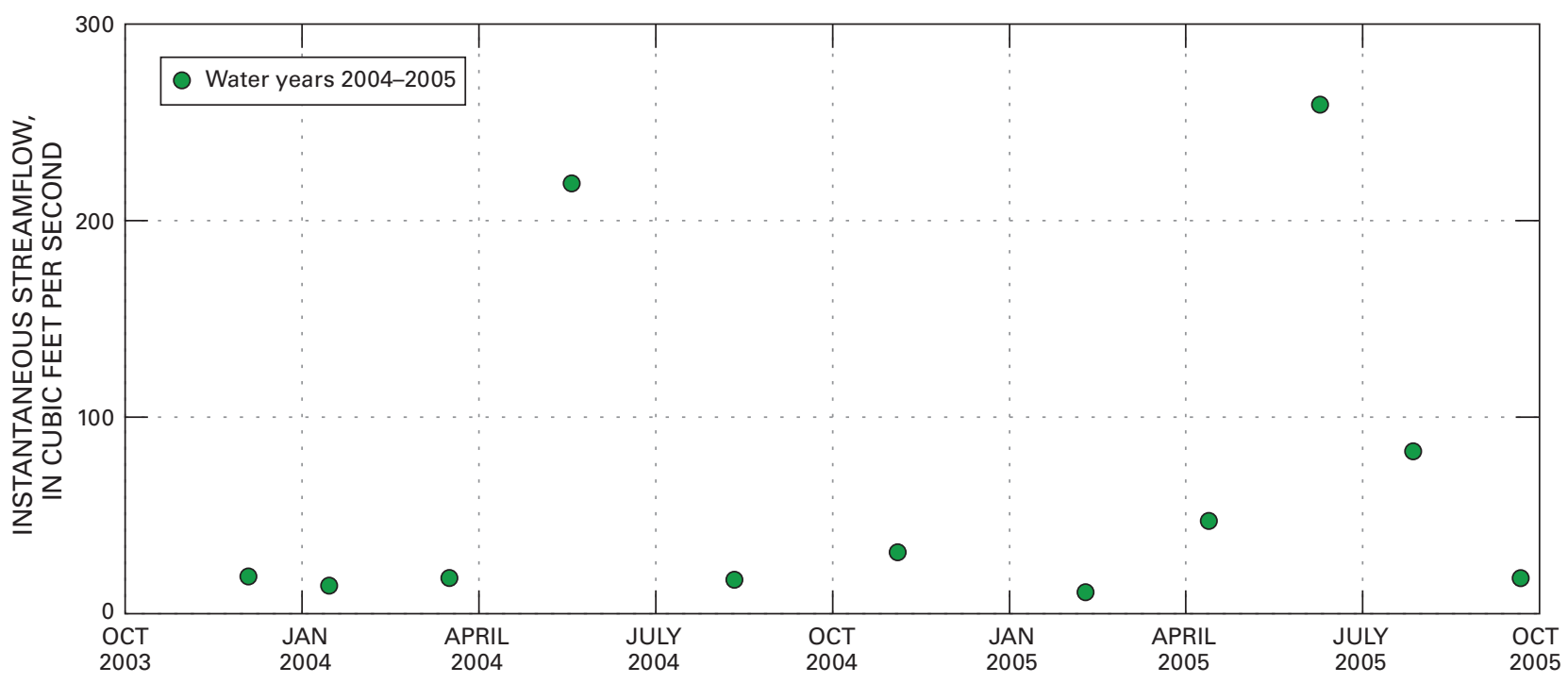

Figure 14. Time distribution and streamflow of water-quality samples for for Ohio Creek near Baldwin. 

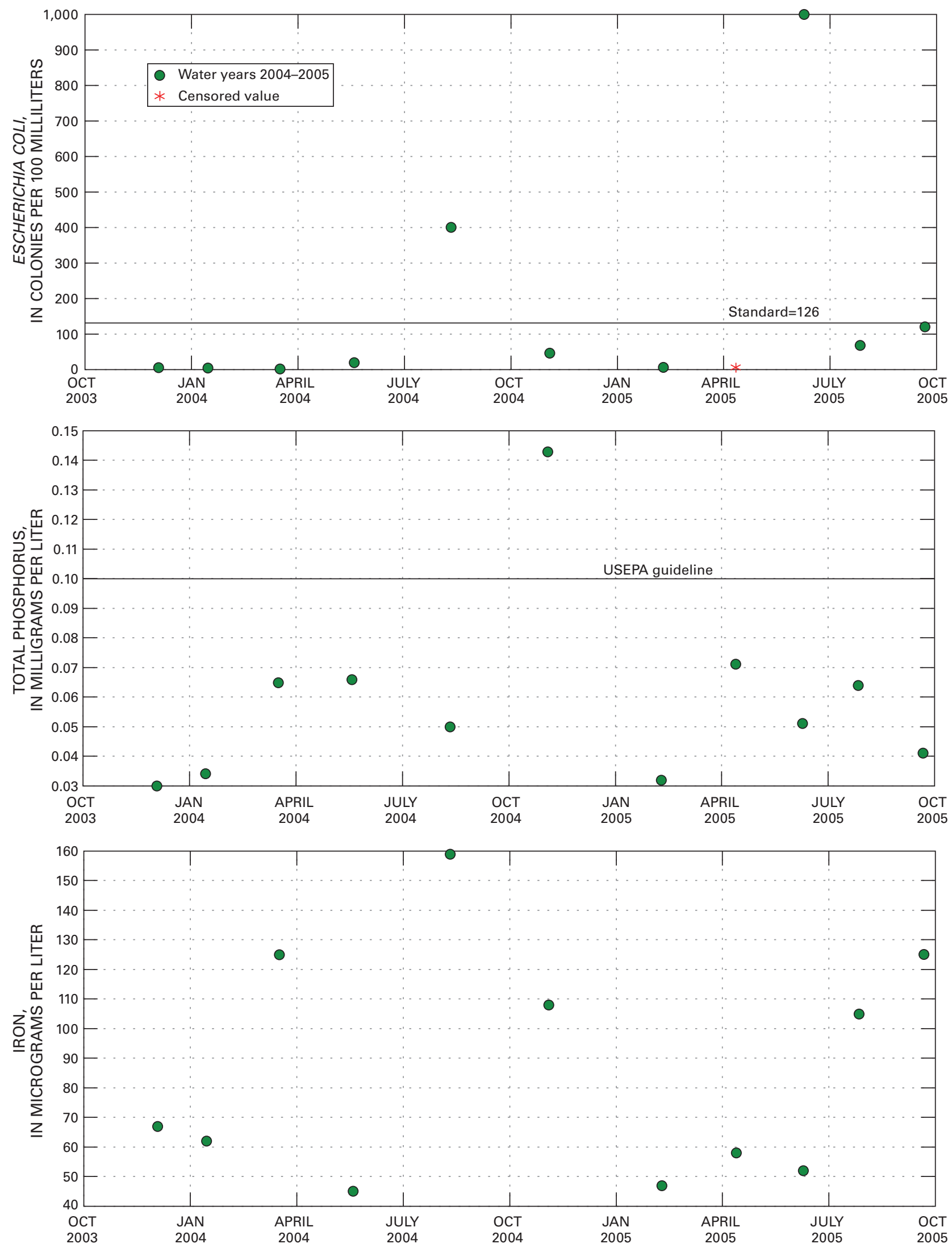

Figure 15. Distribution of selected water-quality constituents relative to time for Ohio Creek near Baldwin. 
Table 8. Summary of measured constituents and properties for Ohio Creek near Baldwin station 09113500

$\left[\mathrm{mg} / \mathrm{L}\right.$, milligrams per liter; $\mu \mathrm{S} / \mathrm{cm}$, microsiemens per centimeter at $25^{\circ}$ Celsius; $\mu \mathrm{g} / \mathrm{L}$, micrograms per liter; ${ }^{\circ} \mathrm{C}$, degrees Celsius; N/A, not applicable; NTU, nephelometric turbidity units; mL, milliliters; $\mathrm{LRL}$, laboratory reporting level]

\begin{tabular}{|c|c|c|c|c|c|c|c|c|c|c|c|c|}
\hline \multirow[b]{2}{*}{$\begin{array}{l}\text { Constituent } \\
\text { or property }\end{array}$} & \multirow[b]{2}{*}{ Units } & \multirow{2}{*}{$\begin{array}{c}\text { Period } \\
\text { (water year) }\end{array}$} & \multirow{2}{*}{$\begin{array}{l}\text { Number of } \\
\text { samples }\end{array}$} & \multirow{2}{*}{$\begin{array}{l}\text { Number of } \\
\text { censored } \\
\text { values }\end{array}$} & \multirow[b]{2}{*}{ Median' } & \multicolumn{2}{|c|}{ Maximum } & \multirow[b]{2}{*}{$\begin{array}{c}\text { 85th } \\
\text { percentile }^{1}\end{array}$} & \multicolumn{2}{|c|}{ Standard $^{2}$} & \multirow[b]{2}{*}{ Trend $^{3}$} & \multirow[b]{2}{*}{ Comment } \\
\hline & & & & & & Value & Date & & Value & $\begin{array}{c}\text { Number of } \\
\text { exceedances }\end{array}$ & & \\
\hline Dissolved oxygen & $\mathrm{mg} / \mathrm{L}$ & $2004-2005$ & 11 & 0 & 9.5 & 10.5 & $04 / 13 / 05$ & 10.1 & 6 & 0 & N/A & $\begin{array}{l}\text { Minimum }=7.3 \\
15 \text { th percentile }=8.5\end{array}$ \\
\hline $\mathrm{pH}$ & $\begin{array}{l}\text { Standard } \\
\text { units }\end{array}$ & 2004-2005 & 11 & 0 & 7.8 & 8.2 & $09 / 21 / 05$ & 8.0 & $6.5-9.0$ & 0 & N/A & $\begin{array}{l}\text { Minimum }=7.2 \\
15 \text { th percentile }=7.4\end{array}$ \\
\hline Specific conductance & $\mu \mathrm{S} / \mathrm{cm}$ & 2004-2005 & 11 & 0 & 134 & 213 & $04 / 13 / 05$ & 203 & None & N/A & N/A & \\
\hline Temperature & ${ }^{\circ} \mathrm{C}$ & 2004-2005 & 11 & 0 & 2 & 15.7 & $07 / 27 / 05$ & 10.9 & 20 & 0 & N/A & \\
\hline Ammonia & $\mathrm{mg} / \mathrm{L}$ & 2004-2005 & 11 & 9 & 0 & 0.008 & $09 / 21 / 05$ & 0.007 & None & N/A & N/A & Current LRL $=0.01$ \\
\hline $\begin{array}{l}\text { Un-ionized ammonia } \\
\text { (computed) }\end{array}$ & $\mathrm{mg} / \mathrm{L}$ & 2004-2005 & 11 & 9 & 0 & 0.0002 & $09 / 21 / 05$ & 0.00003 & 0.02 & 0 & N/A & \\
\hline $\begin{array}{l}\text { Ammonia plus organic } \\
\text { nitrogen (total) }\end{array}$ & $\mathrm{mg} / \mathrm{L}$ & 2004-2005 & 11 & 0 & 0.26 & 0.40 & $11 / 04 / 04$ & 0.38 & None & N/A & N/A & \\
\hline Nitrite plus nitrate & $\mathrm{mg} / \mathrm{L}$ & 2004-2005 & 11 & 4 & 0.03 & 0.09 & $01 / 15 / 04$ & 0.08 & ${ }^{6} 10$ & 0 & N/A & Current LRL $=0.016$ \\
\hline Nitrite & $\mathrm{mg} / \mathrm{L}$ & 2004-2005 & 11 & 3 & 0.001 & 0.002 & 02/09/05 & 0.001 & 0.05 & 0 & N/A & Current LRL $=0.002$ \\
\hline Orthophosphate & $\mathrm{mg} / \mathrm{L}$ & 2004-2005 & 11 & 0 & 0.012 & 0.024 & 03/17/04 & 0.019 & None & N/A & N/A & Current LRL $=0.006$ \\
\hline Phosphorus (total) & $\mathrm{mg} / \mathrm{L}$ & 2004-2005 & 11 & 0 & 0.051 & 0.143 & $11 / 04 / 04$ & 0.071 & 0.1 & 1 & N/A & Concern \\
\hline Sulfate & $\mathrm{mg} / \mathrm{L}$ & 2004-2005 & 11 & 0 & 9.8 & 37.9 & 03/17/04 & 34.1 & ${ }^{6} 250$ & 0 & N/A & \\
\hline Iron & $\mu \mathrm{g} / \mathrm{L}$ & 2004-2005 & 11 & 0 & 67 & 159 & $08 / 11 / 04$ & 125 & 6300 & 0 & N/A & \\
\hline Sediment & $\mathrm{mg} / \mathrm{L}$ & 2004-2005 & 11 & 0 & 7 & 74 & $11 / 04 / 04$ & 53 & None & & N/A & \\
\hline Turbidity & NTU & 2004-2005 & 11 & 0 & 4.6 & 8.7 & 04/13/05 & 6.6 & None & & N/A & \\
\hline Escherichia coli & $\begin{array}{l}\text { Colonies } \\
\text { per } 100 \mathrm{~mL}\end{array}$ & 2004-2005 & 11 & 1 & 19 & 1,000 & 06/09/05 & $\left({ }^{7}\right)$ & 126 & 2 & N/A & Geometric mean $=22$ \\
\hline
\end{tabular}

${ }^{1}$ Censored values were replaced with 0 to compute median and 85th percentiles (coliform censored values replaced with 1). See "Definitions of Terms" section.

${ }^{2}$ Colorado Department of Public Health and Environment, classification and numeric standards for Gunnison and Lower Dolores River Basins (2006); and USEPA, Quality criteria for water (1986).

${ }^{3}$ Insufficient data for trend analysis.

${ }^{4}$ Multiple dates for maximum.

${ }^{5}$ Statistic cannot be computed due to number of censored values or insufficient data.

${ }^{6}$ Instream standard for nitrate, WS standard for sulfate and iron.

${ }^{7}$ Use geometric mean for comparison to standard. 


\section{Ohio Creek above Mouth, near Gunnison, CO}

Current Reason for Inclusion: This station identifies the contributions of the Ohio Creek Basin, which includes development and historical mining. Improves the spatial distribution of the network. Long-term monitoring.

\section{General Station Information:}

Location: County Road 48 bridge, 1.1 miles upstream from the confluence with the Gunnison River, and 3.1 miles north of Gunnison. The gage is on the left bank.

Station Type: USGS water quality and streamflow gaging

Latitude: 383516

Longitude: 1065551
Drainage area: $161 \mathrm{mi}^{2}$

Stream segment: 16

HUC: 14020002

\section{USGS Data Summary:}

Period of Record:

Water quality: October 1996-September 2005

Data prior to September 1998 published as station 383516106555000

Streamflow gaging: December 1998-September 2005

General Chemistry:

Water type: Calcium carbonate

Hardness: Moderately hard

pH: Low concern

Dissolved oxygen: Low concern

Nutrients: Total phosphorus: Concern

E. coli: Low concern

Trace Elements/Metals: No data

Other constituents of concern: None

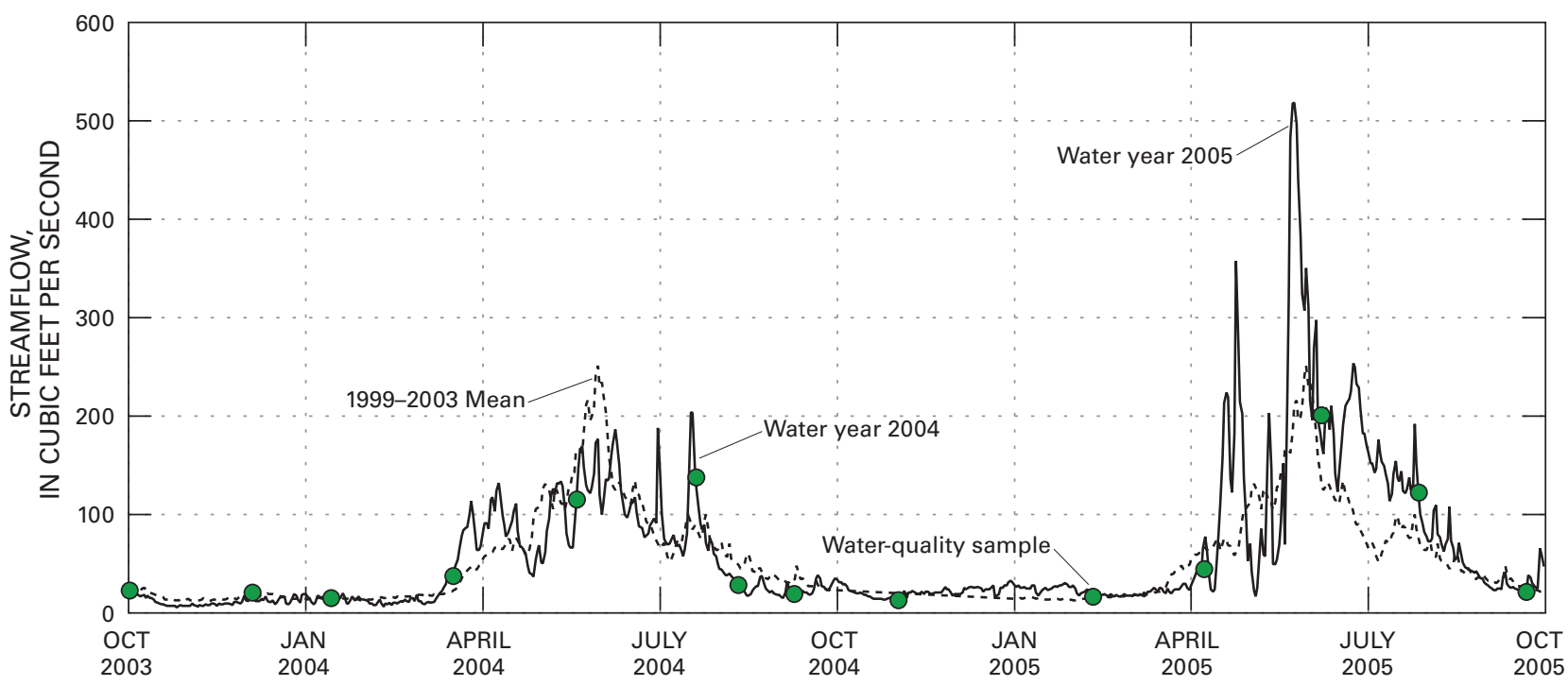

Figure 16. Daily mean streamflow and time distribution of water-quality samples for Ohio Creek above mouth near Gunnison. 

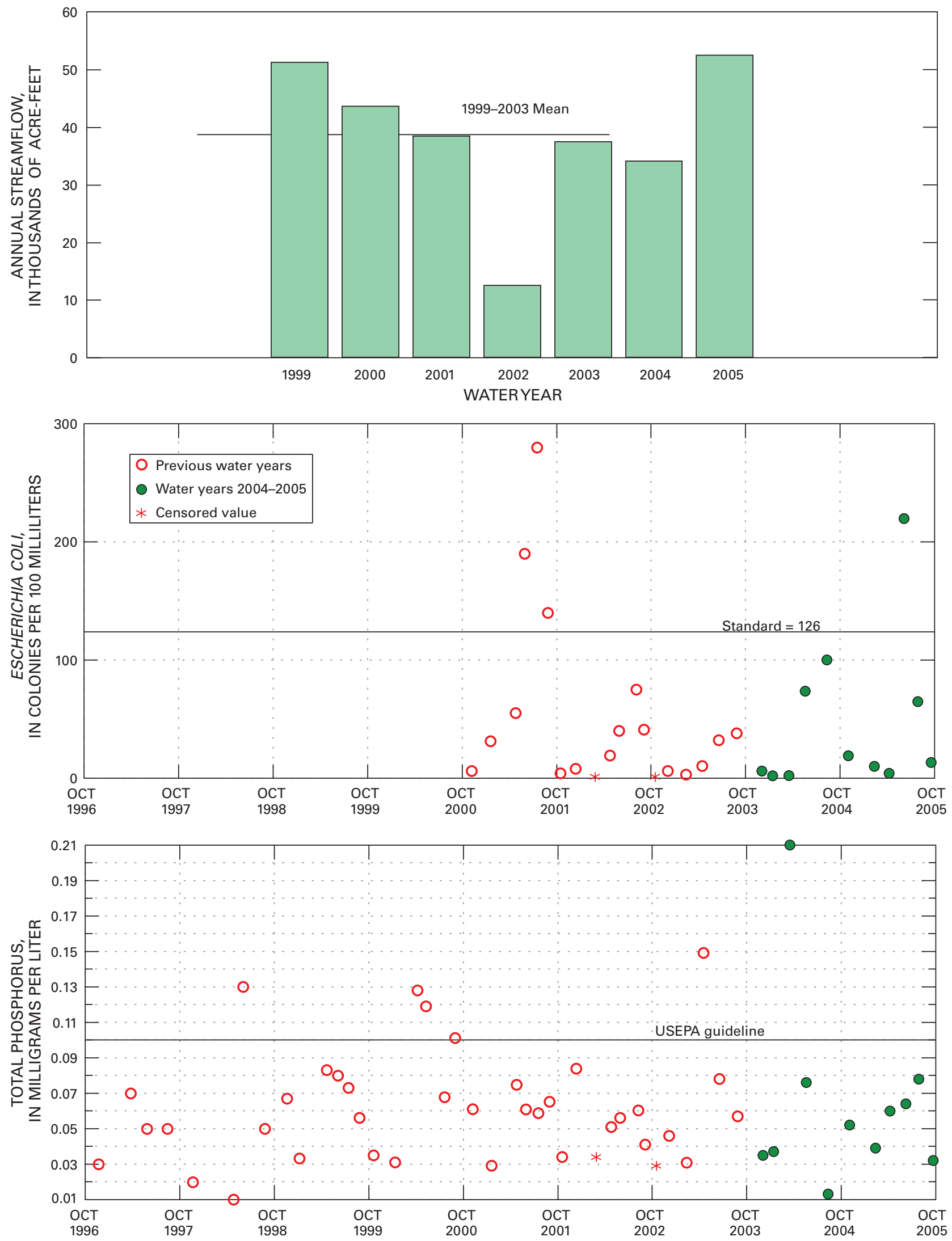

Figure 17. Annual streamflow and distribution of selected water-quality constituents relative to time for Ohio Creek above mouth near Gunnison. 
Table 9. Summary of measured constituents and properties for Ohio Creek above mouth station 09113980.

$\left[\mathrm{mg} / \mathrm{L}\right.$, milligrams per liter; $\mu \mathrm{S} / \mathrm{cm}$, microsiemens per centimeter at $25^{\circ}$ Celsius; $\mu \mathrm{g} / \mathrm{L}$, micrograms per liter; ${ }^{\circ} \mathrm{C}$, degrees Celsius; N/A, not applicable; $\mathrm{mL}$, milliliters; $\mathrm{LRL}$, laboratory reporting level; BOD, biological oxygen demand]

\begin{tabular}{|c|c|c|c|c|c|c|c|c|c|c|c|c|}
\hline \multirow{2}{*}{$\begin{array}{l}\text { Constituent } \\
\text { or property }\end{array}$} & \multirow[b]{2}{*}{ Units } & \multirow{2}{*}{$\begin{array}{c}\text { Period } \\
\text { (water year) }\end{array}$} & \multirow{2}{*}{$\begin{array}{l}\text { Number of } \\
\text { samples }\end{array}$} & \multirow{2}{*}{$\begin{array}{c}\text { Number of } \\
\text { censored } \\
\text { values }\end{array}$} & \multirow[b]{2}{*}{ Median' } & \multicolumn{2}{|c|}{ Maximum } & \multirow{2}{*}{$\begin{array}{c}\text { 85th } \\
\text { percentile }^{1}\end{array}$} & \multicolumn{2}{|c|}{ Standard $^{2}$} & \multirow[b]{2}{*}{ Trend $^{3}$} & \multirow[b]{2}{*}{ Comment } \\
\hline & & & & & & Value & Date & & Value & $\begin{array}{c}\text { Number of } \\
\text { exceedances }\end{array}$ & & \\
\hline \multirow[t]{2}{*}{ Dissolved oxygen } & $\mathrm{mg} / \mathrm{L}$ & 2004-2005 & 11 & 0 & 8.9 & 11.0 & $04 / 08 / 05$ & 10.3 & 6 & 0 & None & Minimum=6.7 \\
\hline & & $1997-2003$ & 39 & 0 & 8.7 & 11.0 & ${ }^{4} 11 / 19 / 97$ & & & 0 & & 15 th percentile $=7.5$ \\
\hline \multirow[t]{2}{*}{$\mathrm{pH}$} & Standard & 2004-2005 & 11 & 0 & 8.1 & 8.5 & $11 / 02 / 04$ & 8.3 & $6.5-9.0$ & 0 & None & Minimum=7.6 \\
\hline & units & 1997-2003 & 39 & 0 & 8.1 & 8.6 & 09/04/02 & & & 0 & & 15 th percentile $=7.9$ \\
\hline \multirow[t]{2}{*}{ Specific conductance } & $\mu \mathrm{S} / \mathrm{cm}$ & 2004-2005 & 14 & 0 & 251 & 359 & $11 / 02 / 04$ & 339 & None & N/A & None & \\
\hline & & 1997-2003 & 61 & 0 & 223 & 438 & $10 / 02 / 02$ & & & N/A & & \\
\hline \multirow[t]{2}{*}{ Temperature } & ${ }^{\circ} \mathrm{C}$ & 2004-2005 & 14 & 0 & 11.8 & 18.8 & $07 / 20 / 04$ & 17.0 & 20 & 0 & N/A & \\
\hline & & 1997-2003 & 61 & 0 & 8.5 & 22.5 & $07 / 16 / 02$ & & & 2 & & \\
\hline \multirow[t]{2}{*}{ Ammonia } & $\mathrm{mg} / \mathrm{L}$ & 2004-2005 & 11 & 4 & 0.005 & 0.080 & $03 / 17 / 04$ & 0.010 & None & N/A & $\left({ }^{5}\right)$ & Current LRL $=0.01$ \\
\hline & & 1997-2003 & 39 & 19 & 0.002 & 0.030 & $04 / 29 / 98$ & & & N/A & & \\
\hline \multirow{2}{*}{$\begin{array}{l}\text { Un-ionized ammonia } \\
\text { (computed) }\end{array}$} & $\mathrm{mg} / \mathrm{L}$ & 2004-2005 & 11 & 4 & 0.00005 & 0.00089 & $03 / 17 / 04$ & 0.00024 & 0.02 & 0 & $\left({ }^{5}\right)$ & \\
\hline & & 1997-2003 & 39 & 19 & 0.00003 & 0.00131 & $08 / 25 / 99$ & & & 0 & & \\
\hline \multirow{2}{*}{$\begin{array}{l}\text { Ammonia plus organic } \\
\text { nitrogen (total) }\end{array}$} & $\mathrm{mg} / \mathrm{L}$ & 2004-2005 & 11 & 0 & 0.25 & 0.93 & $03 / 17 / 04$ & 0.43 & None & N/A & $\left({ }^{5}\right)$ & Current LRL $=0.1$ \\
\hline & & 1997-2003 & 39 & 4 & 0.23 & 0.59 & $04 / 16 / 03$ & & & N/A & & \\
\hline \multirow{2}{*}{ Nitrite plus nitrate } & $\mathrm{mg} / \mathrm{L}$ & 2004-2005 & 11 & 4 & 0.018 & 0.10 & $03 / 17 / 04$ & 0.06 & ${ }^{6} 10$ & 0 & $\left({ }^{5}\right)$ & Current LRL $=0.016$ \\
\hline & & 1997-2003 & 39 & 10 & 0.013 & 0.17 & $04 / 16 / 03$ & & & 0 & & \\
\hline \multirow[t]{2}{*}{ Nitrite } & $\mathrm{mg} / \mathrm{L}$ & 2004-2005 & 11 & 1 & 0.001 & 0.003 & $03 / 17 / 04$ & 0.002 & 0.05 & 0 & $\left({ }^{5}\right)$ & Current LRL $=0.004$ \\
\hline & & $1997-2003$ & 39 & 25 & 0 & 0.030 & $11 / 22 / 96$ & & & 0 & & \\
\hline \multirow[t]{2}{*}{ Orthophosphate } & $\mathrm{mg} / \mathrm{L}$ & 2004-2005 & 11 & 0 & 0.017 & 0.080 & 03/17/04 & 0.029 & None & N/A & None & \\
\hline & & $1997-2003$ & 39 & 0 & 0.020 & 0.046 & $04 / 07 / 00$ & & & N/A & & \\
\hline \multirow[t]{2}{*}{ Phosphorus (total) } & $\mathrm{mg} / \mathrm{L}$ & 2004-2005 & 11 & 0 & 0.052 & 0.210 & $03 / 17 / 04$ & 0.083 & 0.1 & 1 & None & Concern \\
\hline & & $1997-2003$ & 39 & 0 & 0.057 & 0.149 & $04 / 16 / 03$ & & & 5 & & \\
\hline \multirow[t]{2}{*}{ Escherichia coli } & Colonies & 2004-2005 & 11 & 0 & 13 & 220 & $06 / 07 / 05$ & $\left({ }^{7}\right)$ & 126 & 1 & $\left({ }^{5}\right)$ & Geometric mean $=18$ \\
\hline & per $100 \mathrm{~mL}$ & 2001-2003 & 19 & 2 & 31 & 280 & 07/19/01 & & & 3 & & \\
\hline \multirow[t]{2}{*}{ BOD } & $\mathrm{mg} / \mathrm{L}$ & 2004-2005 & 11 & 7 & 0 & 4.0 & $03 / 17 / 04$ & 1.6 & None & N/A & $\left({ }^{5}\right)$ & \\
\hline & & $1997-2003$ & 30 & 19 & 0 & 2.9 & $04 / 07 / 00$ & & & N/A & & \\
\hline
\end{tabular}

'Censored values were replaced with 0 to compute median and 85th percentiles (coliform censored values replaced with 1). See "Definitions of Terms" section.

${ }^{2}$ Colorado Department of Public Health and Environment, classification and numeric standards for Gunnison and Lower Dolores River Basins (2006); and USEPA, Quality criteria for water (1986).

${ }^{3}$ Period of record for trend analysis is water year 1997-water year 2005; flow-adjusted seasonal Kendall method, Helsel and Hirsch, Statistical Methods in Water Resources (1993).

${ }^{4}$ Multiple dates for maximum.

${ }^{5}$ Statistic cannot be computed due to number of censored values or insufficient data.

${ }^{6}$ Instream standard for nitrate.

${ }^{7}$ Use geometric mean for comparison to standard. 


\section{Gunnison River near Gunnison, CO}

Current Reason for Inclusion: This station is upstream from the City of Gunnison treatment-plant discharge and is part of the rotational group of stations.

\section{General Station Information:}

Location: 0.7 mile downstream from Antelope Creek and 1.2 miles west of Gunnison. The gage is on the right bank.

Station Type: USGS water quality and streamflow gaging

Latitude: 383231

Longitude: 1065657

\section{Drainage area: $1,012 \mathrm{mi}^{2}$}

Stream segment: 14
HUC: 14020002

\section{USGS Data Summary:}

Period of Record:

Water quality: April 1995-September 2005

Streamflow gaging: October 1944-September 2005

General Chemistry:

Water type: Calcium carbonate

Hardness: Moderately hard

pH: Low concern

Dissolved oxygen: Low concern

Nutrients: Low concern

E. coli: Low concern

Trace Elements/Metals: No data

Other constituents of concern: None

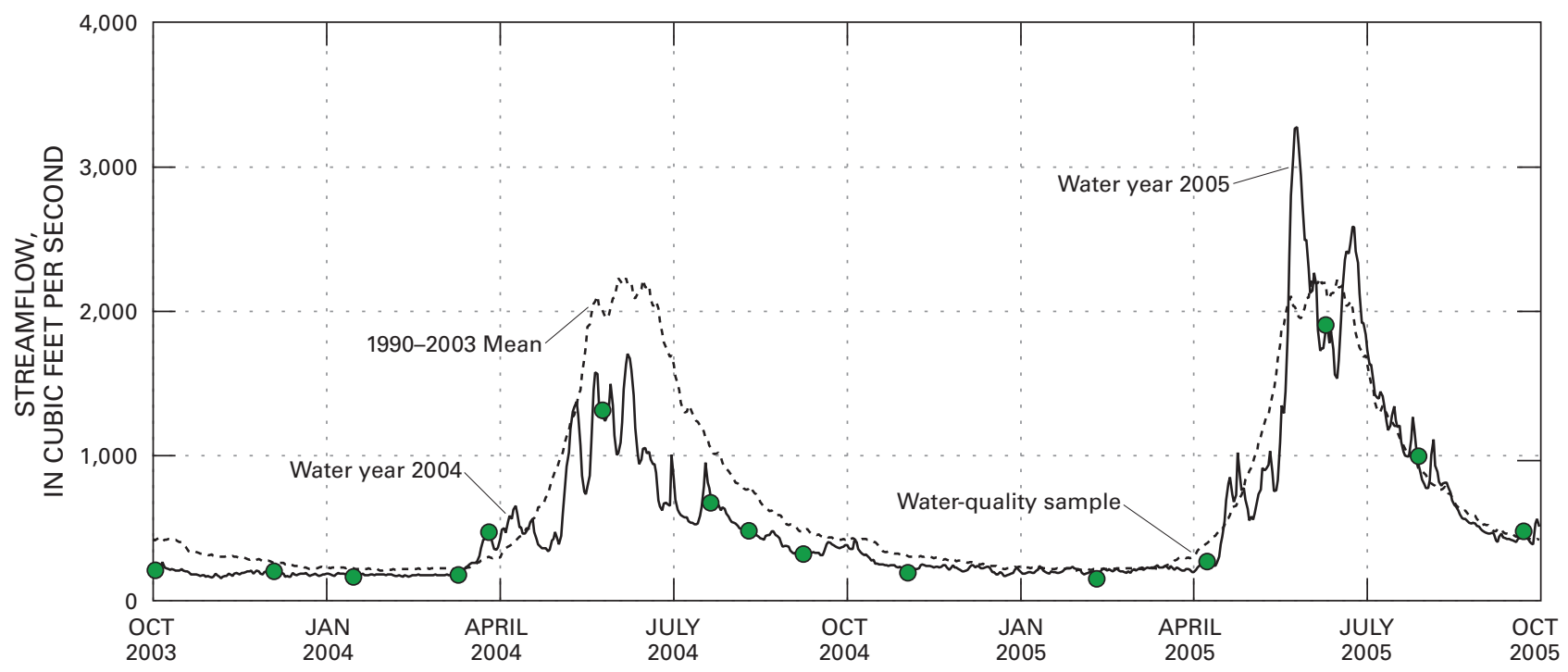

Figure 18. Daily mean streamflow and time distribution of water-quality samples for Gunnison River at Gunnison. 

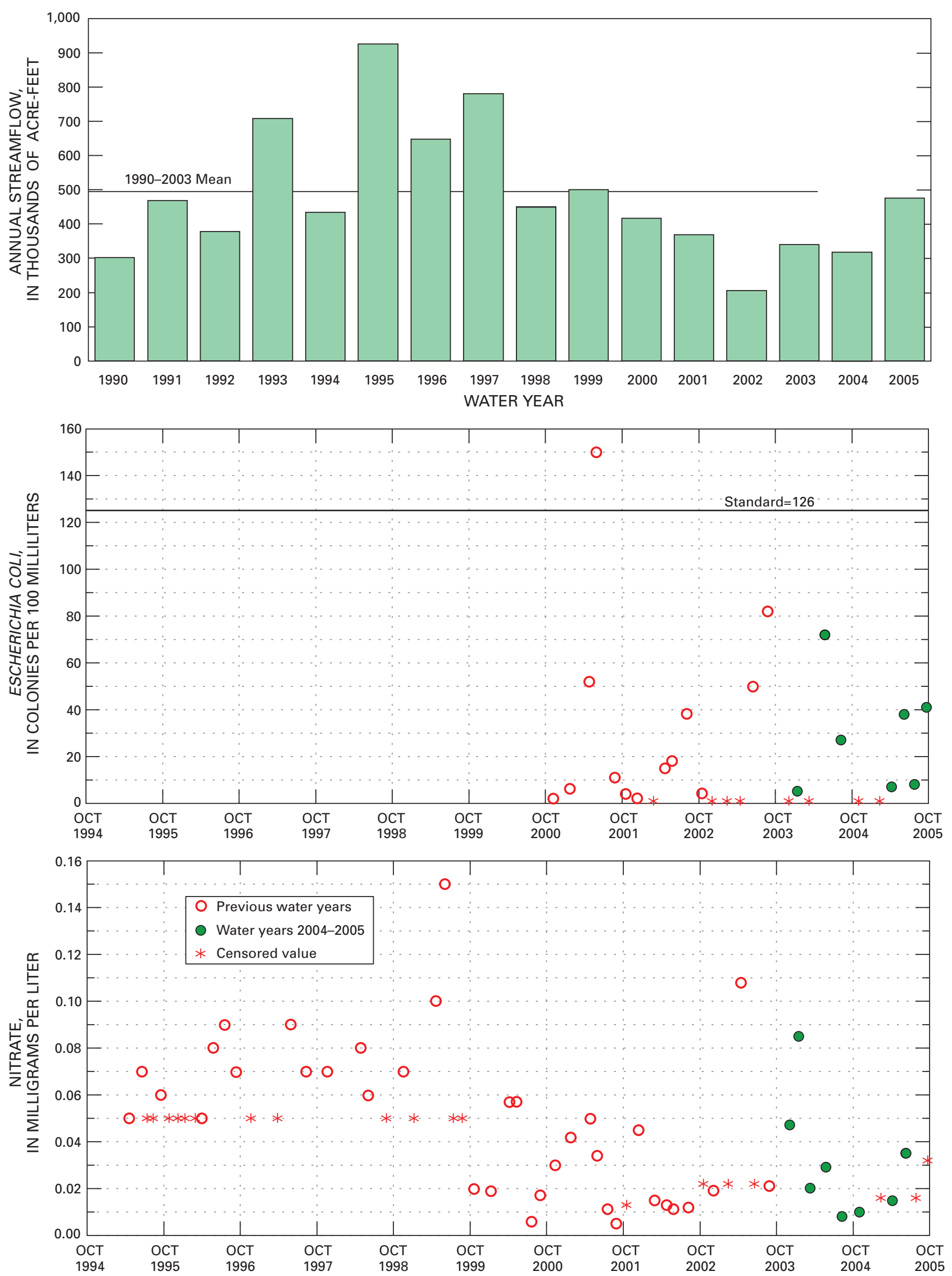

Figure 19. Annual streamflow and distribution of selected water-quality constituents relative to time for Gunnison River at Gunnison. 
Table 10. Summary of measured constituents and properties for Gunnison River near Gunnison station 09114500.

[mg/L, milligrams per liter; $\mu \mathrm{S} / \mathrm{cm}$, microsiemens per centimeter at $25^{\circ}$ Celsius; $\mu \mathrm{g} / \mathrm{L}$, micrograms per liter; ${ }^{\circ} \mathrm{C}$, degrees Celsius; N/A, not applicable; $\mathrm{mL}$, milliliters; $\mathrm{LRL}$, laboratory reporting level; BOD, biological oxygen demand]

\begin{tabular}{|c|c|c|c|c|c|c|c|c|c|c|c|c|}
\hline \multirow{2}{*}{$\begin{array}{l}\text { Constituent } \\
\text { or property }\end{array}$} & \multirow[b]{2}{*}{ Units } & \multirow{2}{*}{$\begin{array}{c}\text { Period } \\
\text { (water year) }\end{array}$} & \multirow{2}{*}{$\begin{array}{l}\text { Number of } \\
\text { samples }\end{array}$} & \multirow{2}{*}{$\begin{array}{l}\text { Number of } \\
\text { censored } \\
\text { values }\end{array}$} & \multirow[b]{2}{*}{ Median $^{1}$} & \multicolumn{2}{|c|}{ Maximum } & \multirow{2}{*}{$\begin{array}{c}\text { 85th } \\
\text { percentile }\end{array}$} & \multicolumn{2}{|c|}{ Standard ${ }^{2}$} & \multirow[b]{2}{*}{ Trend $^{3}$} & \multirow[b]{2}{*}{ Comment } \\
\hline & & & & & & Value & Date & & Value & $\begin{array}{c}\text { Number of } \\
\text { exceedances }\end{array}$ & & \\
\hline \multirow[t]{2}{*}{ Dissolved oxygen } & $\mathrm{mg} / \mathrm{L}$ & 2004-2005 & 11 & 0 & 9.8 & 13.4 & $02 / 10 / 05$ & 11.1 & 6 & 0 & None & Minimum=7.7 \\
\hline & & 1995-2003 & 51 & 0 & 9.4 & 13.0 & $12 / 04 / 02$ & & & 0 & & 15 th percentile $=8.2$ \\
\hline \multirow[t]{2}{*}{$\mathrm{pH}$} & Standard & 2004-2005 & 11 & 0 & 8.2 & 8.8 & $02 / 10 / 05$ & 8.4 & $6.5-9.0$ & 0 & None & Minimum=7.6 \\
\hline & units & 1995-2003 & 51 & 0 & 8.2 & 8.8 & $04 / 16 / 03$ & & & 0 & & 15 th percentile $=8.0$ \\
\hline \multirow[t]{2}{*}{ Specific conductance } & $\mu \mathrm{S} / \mathrm{cm}$ & 2004-2005 & 15 & 0 & 230 & 269 & $11 / 02 / 04$ & 241 & None & N/A & Up & \\
\hline & & 1995-2003 & 105 & 0 & 212 & 330 & $06 / 17 / 03$ & & & N/A & & \\
\hline \multirow[t]{2}{*}{ Temperature } & ${ }^{\circ} \mathrm{C}$ & 2004-2005 & 15 & 0 & 9.8 & 17.1 & $07 / 21 / 04$ & 13.4 & 20 & 0 & N/A & \\
\hline & & 1995-2003 & 105 & 0 & 7.4 & 17.0 & 08/28/03 & & & 0 & & \\
\hline \multirow[t]{2}{*}{ Ammonia } & $\mathrm{mg} / \mathrm{L}$ & 2004-2005 & 11 & 9 & 0 & 0.006 & $04 / 08 / 05$ & 0.020 & None & N/A & $\left({ }^{5}\right)$ & Current LRL $=0.01$ \\
\hline & & $1995-2003$ & 51 & 33 & 0 & 0.050 & 06/04/98 & & & N/A & & \\
\hline \multirow{2}{*}{$\begin{array}{l}\text { Un-ionized ammonia } \\
\text { (computed) }\end{array}$} & $\mathrm{mg} / \mathrm{L}$ & 2004-2005 & 11 & 9 & 0 & 0.00249 & $02 / 10 / 05$ & 0.00034 & 0.02 & 0 & $\left({ }^{5}\right)$ & \\
\hline & & $1995-2003$ & 51 & 33 & 0 & 0.00121 & $11 / 21 / 96$ & & & 0 & & \\
\hline \multirow{2}{*}{$\begin{array}{l}\text { Ammonia plus organic } \\
\text { nitrogen (total) }\end{array}$} & $\mathrm{mg} / \mathrm{L}$ & 2004-2005 & 11 & 0 & 0.14 & 0.25 & $05 / 25 / 04$ & 0.22 & None & N/A & $\left({ }^{5}\right)$ & \\
\hline & & $1995-2003$ & 51 & 19 & 0.09 & 0.46 & $04 / 27 / 01$ & & & N/A & & \\
\hline \multirow{2}{*}{ Nitrite plus nitrate } & $\mathrm{mg} / \mathrm{L}$ & 2004-2005 & 11 & 3 & 0.015 & 0.085 & $01 / 15 / 04$ & 0.07 & ${ }^{6} 10$ & 0 & $\left({ }^{5}\right)$ & Current LRL $=0.016$ \\
\hline & & $1995-2003$ & 51 & 16 & 0.019 & 0.150 & 06/03/99 & & & 0 & & \\
\hline \multirow[t]{2}{*}{ Nitrite } & $\mathrm{mg} / \mathrm{L}$ & 2004-2005 & 11 & 4 & 0.001 & 0.001 & ${ }^{4} 09 / 22 / 08$ & 0.001 & 0.05 & 0 & $\left({ }^{5}\right)$ & Current LRL $=0.002$ \\
\hline & & $1995-2003$ & 51 & 42 & 0 & 0.011 & 06/04/98 & & & 0 & & \\
\hline \multirow[t]{2}{*}{ Orthophosphate } & $\mathrm{mg} / \mathrm{L}$ & 2004-2005 & 11 & 7 & 0 & 0.005 & $04 / 08 / 05$ & 0.010 & None & N/A & $\left({ }^{5}\right)$ & Current LRL $=0.006$ \\
\hline & & $1995-2003$ & 51 & 34 & 0 & 0.030 & ${ }^{4} 04 / 20 / 96$ & & & N/A & & \\
\hline \multirow[t]{2}{*}{ Phosphorus (total) } & $\mathrm{mg} / \mathrm{L}$ & 2004-2005 & 11 & 0 & 0.022 & 0.041 & 08/10/04 & 0.030 & 0.1 & 0 & $\left(5^{5}\right)$ & \\
\hline & & $1995-2003$ & 50 & 11 & 0.014 & 0.120 & $06 / 19 / 95$ & & & 1 & & \\
\hline \multirow[t]{2}{*}{ Escherichia coli } & Colonies & 2004-2005 & 11 & 4 & 7 & 72 & $05 / 25 / 04$ & $\left({ }^{7}\right)$ & 126 & 0 & $\left({ }^{5}\right)$ & Geometric mean $=7$ \\
\hline & per $100 \mathrm{~mL}$ & 2001-2003 & 17 & 4 & 6 & 150 & $05 / 30 / 01$ & & & 1 & & \\
\hline \multirow[t]{2}{*}{ BOD } & $\mathrm{mg} / \mathrm{L}$ & $2004-2005$ & 10 & 8 & 0 & 2.6 & $02 / 10 / 05$ & 1.2 & None & N/A & $(5)$ & \\
\hline & & $1995-2003$ & 38 & 31 & 0 & 2.6 & $09 / 21 / 95$ & & & N/A & & \\
\hline
\end{tabular}

${ }^{1}$ Censored values were replaced with 0 to compute median and 85th percentiles (coliform censored values replaced with 1). See "Definitions of Terms" section.

${ }^{2}$ Colorado Department of Public Health and Environment, classification and numeric standards for Gunnison and Lower Dolores River Basins (2006); and USEPA, Quality criteria for water (1986).

${ }^{3}$ Period of record for trend analysis is water year 1997-water year 2003; flow-adjusted seasonal Kendall method, Helsel and Hirsch, Statistical Methods in Water Resources (1993).

${ }^{4}$ Multiple dates for maximum.

${ }^{5}$ Statistic cannot be computed due to number of censored values or insufficient data.

${ }^{6}$ Instream standard for nitrate.

${ }^{7}$ Use geometric mean for comparison to standard. 


\section{Tomichi Creek below Cochetopa Creek, CO}

General Station Information:

Location: One mile downstream from confluence with Cochetopa Creek and 4 miles northwest of Parlin.

Station Type: USGS water quality

Latitude: 383126

Longitude: 1064756
Drainage area: $984 \mathrm{mi}^{2}$

Stream segment: 18

HUC: 14020003

\section{USGS Data Summary:}

Period of Record:

Water quality: November 2000 - September 2005

General Chemistry:

Water type: Major ion data not collected

Hardness: Moderately hard

pH: Low concern

Dissolved oxygen: Low concern

Nutrients:

Phosphorus: Concern

E. coli: Low concern

Trace Elements/Metals: Low concern

Other constituents of concern: None

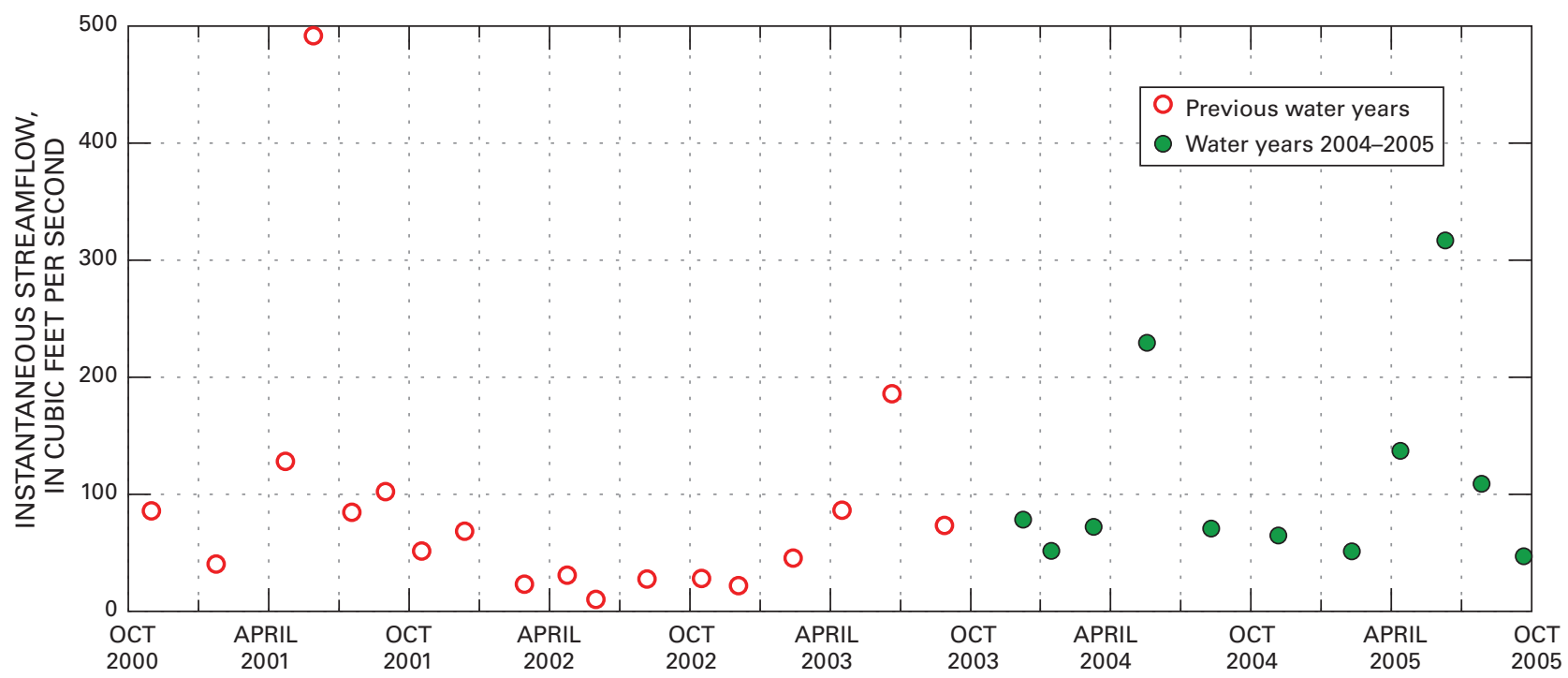

Figure 20. Time distribution and streamflow of water-quality samples for Tomichi Creek below Cochetopa Creek. 

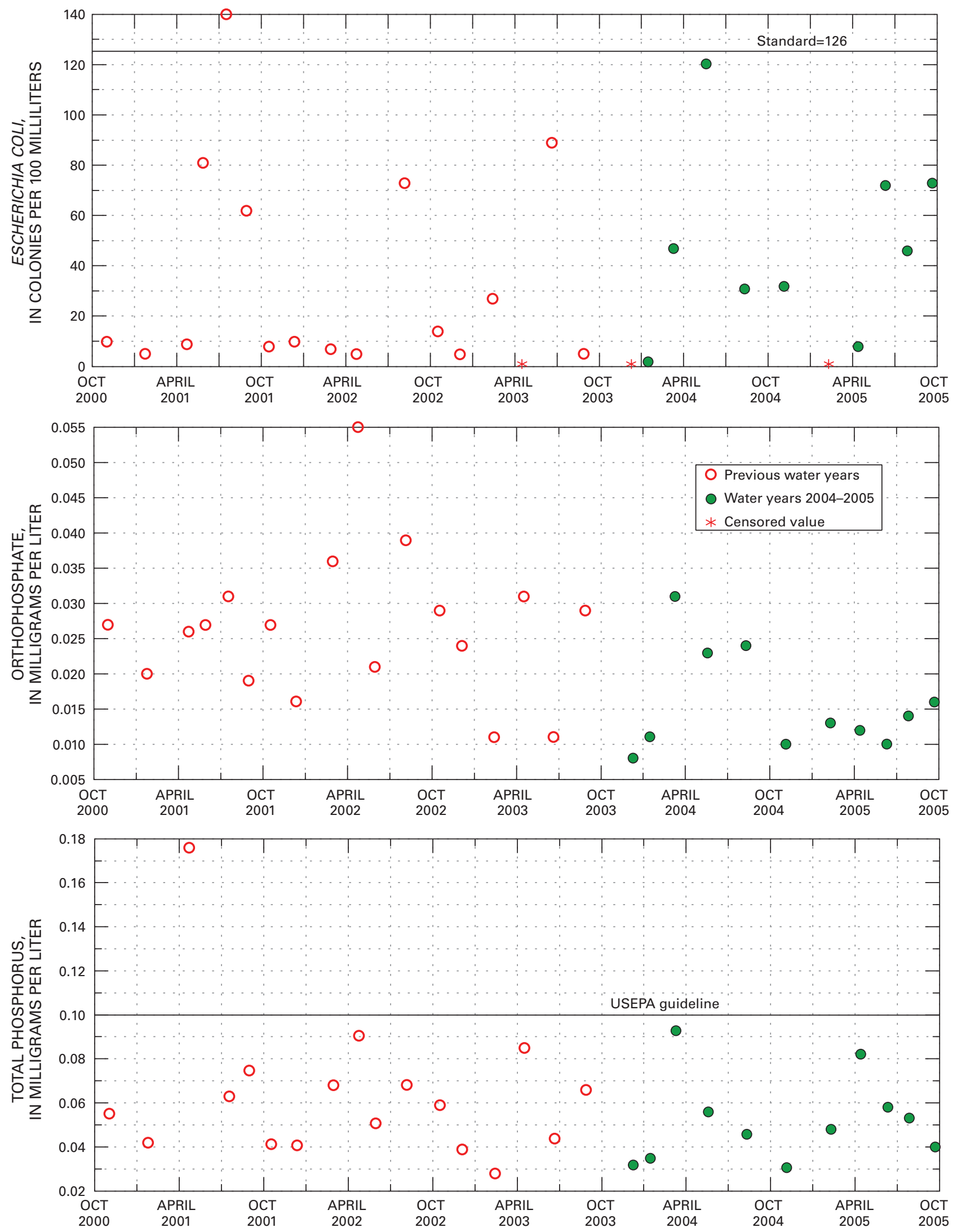

Figure 21. Distribution of selected water-quality constituents relative to time for Tomichi Creek below Cochetopa Creek. 
Table 11. Summary of measured constituents and properties for Tomichi Creek below Cochetopa Creek station 383126106475600.

[mg/L, milligrams per liter; $\mu \mathrm{S} / \mathrm{cm}$, microsiemens per centimeter at $25^{\circ}$ Celsius; $\mu \mathrm{g} / \mathrm{L}$, micrograms per liter; ${ }^{\circ} \mathrm{C}$, degrees Celsius; $\mathrm{CaCO}_{3}$, calcium carbonate; $\mathrm{N} / \mathrm{A}$, not applicable; $\mathrm{NTU}$, nephelometric turbidity units; (ch), chronic standard; mL, milliliters; --, no value; LRL, laboratory reporting level; <, less than]

\begin{tabular}{|c|c|c|c|c|c|c|c|c|c|c|c|c|}
\hline \multirow{2}{*}{$\begin{array}{l}\text { Constituent } \\
\text { or property }\end{array}$} & \multirow[b]{2}{*}{ Units } & \multirow{2}{*}{$\begin{array}{c}\text { Period } \\
\text { (water year) }\end{array}$} & \multirow{2}{*}{$\begin{array}{c}\text { Number of } \\
\text { samples }\end{array}$} & \multirow{2}{*}{$\begin{array}{c}\text { Number of } \\
\text { censored } \\
\text { values }\end{array}$} & \multirow[b]{2}{*}{ Median' } & \multicolumn{2}{|c|}{ Maximum } & \multirow{2}{*}{$\begin{array}{c}\text { 85th } \\
\text { percentile }^{1}\end{array}$} & \multicolumn{2}{|c|}{ Standard $^{2}$} & \multirow[b]{2}{*}{ Trend $^{3}$} & \multirow[b]{2}{*}{ Comment } \\
\hline & & & & & & Value & Date & & Value & $\begin{array}{c}\text { Number of } \\
\text { exceedances }\end{array}$ & & \\
\hline \multirow[t]{2}{*}{ Dissolved oxygen } & $\mathrm{mg} / \mathrm{L}$ & 2004-2005 & 11 & 0 & 9.5 & 11.1 & $08 / 10 / 04$ & 10.7 & 6 & 0 & None & Minimum=7.5 \\
\hline & & 2001-2003 & 17 & 0 & 9.4 & 11.0 & $01 / 24 / 01$ & & & 0 & & 15 th percentile $=8.6$ \\
\hline \multirow[t]{2}{*}{$\mathrm{pH}$} & Standard & 2004-2005 & 11 & 0 & 8.2 & 9.0 & $07 / 27 / 05$ & 8.4 & $6.5-9.0$ & 0 & None & Minimum=7.7 \\
\hline & units & $2001-2003$ & 18 & 0 & 8.2 & 8.8 & 08/06/02 & & & 0 & & 15 th percentile $=7.8$ \\
\hline \multirow[t]{2}{*}{ Specific conductance } & $\mu \mathrm{S} / \mathrm{cm}$ & 2004-2005 & 11 & 0 & 275 & 320 & $09 / 21 / 05$ & 320 & None & N/A & None & \\
\hline & & 2001-2003 & 18 & 0 & 260 & 420 & $05 / 31 / 02$ & & & N/A & & \\
\hline \multirow[t]{2}{*}{ Temperature } & ${ }^{\circ} \mathrm{C}$ & 2004-2005 & 11 & 0 & 7.0 & 21.8 & $07 / 27 / 05$ & 15.5 & 20 & 1 & N/A & \\
\hline & & 2001-2003 & 17 & 0 & 7.5 & 21.4 & 08/06/02 & & & 1 & & \\
\hline \multirow[t]{2}{*}{ Hardness (computed) } & $\mathrm{mg} / \mathrm{L}$ & 2004-2005 & 7 & 0 & 138 & 149 & $11 / 05 / 04$ & 164 & None & N/A & None & \\
\hline & as $\mathrm{CaCO}_{3}$ & $2001-2003$ & 12 & 0 & 125 & 196 & $05 / 31 / 02$ & & & N/A & & \\
\hline \multirow{2}{*}{ Calcium } & $\mathrm{mg} / \mathrm{L}^{3}$ & $2004-2005$ & 7 & 0 & 39 & 42 & $11 / 05 / 04$ & 46 & None & N/A & None & \\
\hline & & 2001-2003 & 12 & 0 & 36 & 54 & $05 / 31 / 02$ & & & N/A & & \\
\hline \multirow[t]{2}{*}{ Magnesium } & $\mathrm{mg} / \mathrm{L}$ & 2004-2005 & 7 & 0 & 10.2 & 11.4 & 06/10/05 & 11.9 & None & N/A & None & \\
\hline & & $2001-2003$ & 12 & 0 & 8.6 & 14.9 & $05 / 31 / 02$ & & & N/A & & \\
\hline \multirow[t]{2}{*}{ Ammonia } & $\mathrm{mg} / \mathrm{L}$ & 2004-2005 & 11 & 7 & 0 & 0.016 & $02 / 09 / 05$ & 0.008 & None & N/A & $\left({ }^{5}\right)$ & Current LRL $=0.01$ \\
\hline & & 2001-2003 & 18 & 13 & 0 & 0.011 & $01 / 24 / 01$ & & & N/A & & \\
\hline \multirow{2}{*}{$\begin{array}{l}\text { Un-ionized ammonia } \\
\text { (computed) }\end{array}$} & $\mathrm{mg} / \mathrm{L}$ & 2004-2005 & 11 & 7 & 0 & 0.00188 & $07 / 27 / 05$ & 0.00007 & 0.02 & 0 & $\left({ }^{5}\right)$ & \\
\hline & & 2001-2003 & 17 & 13 & 0 & 0.00041 & $07 / 18 / 01$ & & & 0 & & \\
\hline \multirow{2}{*}{$\begin{array}{l}\text { Ammonia plus organic } \\
\text { nitrogen (total) }\end{array}$} & $\mathrm{mg} / \mathrm{L}$ & 2004-2005 & 11 & 0 & 0.33 & 0.49 & 05/19/04 & 0.47 & None & N/A & None & \\
\hline & & $2001-2003$ & 17 & 0 & 0.25 & 0.55 & $06 / 20 / 03$ & & & N/A & & \\
\hline \multirow[t]{2}{*}{ Nitrite plus nitrate } & $\mathrm{mg} / \mathrm{L}$ & 2004-2005 & 11 & 8 & 0 & 0.083 & $01 / 15 / 04$ & 0.029 & ${ }^{6} 10$ & 0 & $\left({ }^{5}\right)$ & Current LRL $=0.016$ \\
\hline & & $2001-2003$ & 18 & 10 & 0 & 0.088 & $01 / 24 / 01$ & & & 0 & & \\
\hline \multirow[t]{2}{*}{ Nitrite } & $\mathrm{mg} / \mathrm{L}$ & 2004-2005 & 11 & 4 & 0.001 & 0.003 & $02 / 09 / 05$ & 0.001 & 0.05 & 0 & $\left({ }^{5}\right)$ & Current LRL $=0.002$ \\
\hline & & $2001-2003$ & 18 & 15 & 0 & 0.002 & $02 / 28 / 02$ & & & 0 & & \\
\hline \multirow[t]{2}{*}{ Orthophosphate } & $\mathrm{mg} / \mathrm{L}$ & 2004-2005 & 11 & 0 & 0.013 & 0.031 & $03 / 10 / 04$ & 0.031 & None & N/A & Down & \\
\hline & & 2001-2003 & 18 & 0 & 0.027 & 0.055 & $04 / 24 / 02$ & & & N/A & & \\
\hline \multirow[t]{2}{*}{ Phosphorus (total) } & $\mathrm{mg} / \mathrm{L}$ & 2004-2005 & 11 & 0 & 0.048 & 0.093 & 03/10/04 & 0.082 & 0.1 & 0 & Down & Concern \\
\hline & & $2001-2003$ & 17 & 0 & 0.059 & 0.176 & $04 / 23 / 01$ & & & 1 & & \\
\hline \multirow[t]{2}{*}{ Aluminum } & $\mu \mathrm{g} / \mathrm{L}$ & 2004-2005 & 7 & 0 & 1 & 3 & ${ }^{4} 05 / 19 / 04$ & 2 & 87 (ch) & 0 & $\left({ }^{5}\right)$ & \\
\hline & & 2001-2003 & 12 & 10 & 0 & 2 & ${ }^{4} 08 / 28 / 03$ & & & 0 & & \\
\hline
\end{tabular}


Table 11. Summary of measured constituents and properties for Tomichi Creek below Cochetopa Creek station 383126106475600.-Continued

$\left[\mathrm{mg} / \mathrm{L}\right.$, milligrams per liter; $\mu \mathrm{S} / \mathrm{cm}$, microsiemens per centimeter at $25^{\circ}$ Celsius; $\mu \mathrm{g} / \mathrm{L}$, micrograms per liter; ${ }^{\circ} \mathrm{C}$, degrees Celsius; $\mathrm{CaCO}_{3}$, calcium carbonate; $\mathrm{N} / \mathrm{A}$, not applicable; $\mathrm{NTU}$, nephelometric turbidity units; (ch), chronic standard; mL, milliliters; --, no value; LRL, laboratory reporting level; <, less than]

\begin{tabular}{|c|c|c|c|c|c|c|c|c|c|c|c|c|}
\hline \multirow[b]{2}{*}{$\begin{array}{l}\text { Constituent } \\
\text { or property }\end{array}$} & \multirow[b]{2}{*}{ Units } & \multirow[b]{2}{*}{$\begin{array}{c}\text { Period } \\
\text { (water year) }\end{array}$} & \multirow[b]{2}{*}{$\begin{array}{l}\text { Number of } \\
\text { samples }\end{array}$} & \multirow{2}{*}{$\begin{array}{c}\text { Number of } \\
\text { censored } \\
\text { values }\end{array}$} & \multirow[b]{2}{*}{ Median' } & \multicolumn{2}{|c|}{ Maximum } & \multirow[b]{2}{*}{$\begin{array}{c}\text { 85th } \\
\text { percentile }^{1}\end{array}$} & \multicolumn{2}{|c|}{ Standard $^{2}$} & \multirow[b]{2}{*}{ Trend $^{3}$} & \multirow[b]{2}{*}{ Comment } \\
\hline & & & & & & Value & Date & & Value & $\begin{array}{c}\text { Number of } \\
\text { exceedances }\end{array}$ & & \\
\hline \multirow[t]{2}{*}{ Cadmium } & $\mu \mathrm{g} / \mathrm{L}$ & 2004-2005 & 7 & 5 & 0 & 0.02 & ${ }^{4} 06 / 10 / 05$ & 0 & $2.4(\mathrm{ch})$ & 0 & $\left({ }^{5}\right)$ & Current LRL $=0.4$ \\
\hline & & 2001-2003 & 12 & 12 & 0 & ${ }^{7}<0.2$ & -- & & & 0 & & \\
\hline \multirow[t]{2}{*}{ Copper } & $\mu \mathrm{g} / \mathrm{L}$ & 2004-2005 & 7 & 0 & 0.90 & 1.9 & 06/10/05 & 1.5 & $9.5(\mathrm{ch})$ & 0 & $\left({ }^{5}\right)$ & \\
\hline & & 2001-2003 & 12 & 6 & 0.35 & 1.8 & $05 / 29 / 01$ & & & 0 & & \\
\hline \multirow[t]{2}{*}{ Iron } & $\mu \mathrm{g} / \mathrm{L}$ & 2004-2005 & 7 & 0 & 73 & 87 & $05 / 19 / 04$ & 98 & 300 & 0 & None & Water supply standard \\
\hline & & 2001-2003 & 12 & 0 & 79 & 157 & $04 / 17 / 03$ & & & 0 & & \\
\hline \multirow[t]{2}{*}{ Lead } & $\mu \mathrm{g} / \mathrm{L}$ & 2004-2005 & 7 & 0 & 0.08 & 0.17 & $06 / 10 / 05$ & 0.11 & $2.7(\mathrm{ch})$ & 0 & $\left({ }^{5}\right)$ & \\
\hline & & 2001-2003 & 11 & 11 & 0 & ${ }^{7}<1$ & -- & & & 0 & & \\
\hline \multirow[t]{2}{*}{ Manganese } & $\mu \mathrm{g} / \mathrm{L}$ & 2004-2005 & 7 & 0 & 40 & 76 & $06 / 10 / 05$ & 72 & $1,689(\mathrm{ch})$ & 0 & None & Water supply standard \\
\hline & & 2001-2003 & 12 & 0 & 38 & 117 & $05 / 31 / 02$ & & & 0 & & is 50 \\
\hline \multirow[t]{2}{*}{ Silver } & $\mu \mathrm{g} / \mathrm{L}$ & 2004-2005 & 7 & 7 & 0 & ${ }^{7}<0.2$ & -- & 0 & $0 . .36(\mathrm{ch})$ & 0 & $\left({ }^{5}\right)$ & Current LRL $=0.2$ \\
\hline & & 2001-2003 & 12 & 12 & 0 & ${ }^{7}<0.3$ & -- & & & 0 & & \\
\hline \multirow[t]{2}{*}{ Zinc } & $\mu \mathrm{g} / \mathrm{L}$ & $2004-2005$ & 7 & 0 & 1.2 & 2.2 & $06 / 10 / 05$ & 2.2 & $125(\mathrm{ch})$ & 0 & $\left({ }^{5}\right)$ & \\
\hline & & 2001-2003 & 12 & 9 & 0 & 13 & $04 / 32 / 01$ & & & 0 & & \\
\hline \multirow[t]{2}{*}{ Suspended sediment } & $\mathrm{mg} / \mathrm{L}$ & 2004-2005 & 7 & 0 & 10 & 20 & $04 / 13 / 05$ & 20 & None & N/A & None & \\
\hline & & 2001-2003 & 12 & 0 & 6 & 104 & $04 / 23 / 01$ & & & N/A & & \\
\hline \multirow[t]{2}{*}{ Turbidity } & NTU & 2004-2005 & 7 & 1 & 3 & 8 & $04 / 13 / 05$ & 11 & None & N/A & $\left({ }^{5}\right)$ & \\
\hline & & 2001-2003 & 12 & 0 & 4 & 37 & $04 / 23 / 01$ & & & N/A & & \\
\hline \multirow[t]{2}{*}{ Escherichia coli } & Colonies & $2004-2005$ & 11 & 2 & 32 & 120 & $05 / 19 / 04$ & $\left({ }^{8}\right)$ & 126 & 0 & None & Geometric mean $=15$ \\
\hline & per $100 \mathrm{~mL}$ & 2001-2003 & 17 & 1 & 10 & 140 & $07 / 18 / 01$ & & & 1 & & \\
\hline
\end{tabular}

${ }^{1}$ Censored values were replaced with 0 to compute median and 85th percentiles (coliform censored values replaced with 1). See "Definitions of Terms" section.

${ }^{2}$ Colorado Department of Public Health and Environment, classification and numeric standards for Gunnison and Lower Dolores River Basins (2006); and USEPA, Quality criteria for water (1986).

${ }^{3}$ Period of record for trend analysis is water year 2001-water year 2005; flow-adjusted seasonal Kendall method, Helsel and Hirsch, Statistical Methods in Water Resources (1993).

${ }^{4}$ Multiple dates for maximum.

${ }^{5}$ Statistic cannot be computed due to number of censored values or insufficient data.

${ }^{6}$ Instream standard for nitrate.

${ }^{7}$ All values censored, current LRL used for maximum.

${ }^{8}$ Use geometric mean for comparison to standard. 


\section{Tomichi Creek at Gunnison, CO}

Current Reason for Inclusion: This station represents contribution of entire the Tomichi Creek Basin and allows for Tomichi Creek influences to be separated from those of the Gunnison River. Improves the spatial distribution of the network. Long-term monitoring.

\section{General Station Information:}

Location: 300 feet downstream from highway bridge, 1.8 miles southwest of United States Post Office in Gunnison and 2.0 miles upstream from mouth.

Station Type: USGS water quality and streamflow-gaging site

Latitude: 383118

Longitude: 1065625
Drainage area: $1,061 \mathrm{mi}^{2}$

Stream segment: 18

HUC: 14020003

\section{USGS Data Summary:}

Period of Record:

Water quality:

October 1990-September 1993

April 1995-September 2005

Streamflow gaging: October 1937-September 2005

General Chemistry:

Water type: Calcium carbonate

Hardness: Moderately hard

pH: Low concern

Dissolved oxygen: Low concern

Nutrients:

Total phosphorus: Concern

E. coli: Low concern

Trace Elements/Metals: Low concern

Other constituents of concern: None

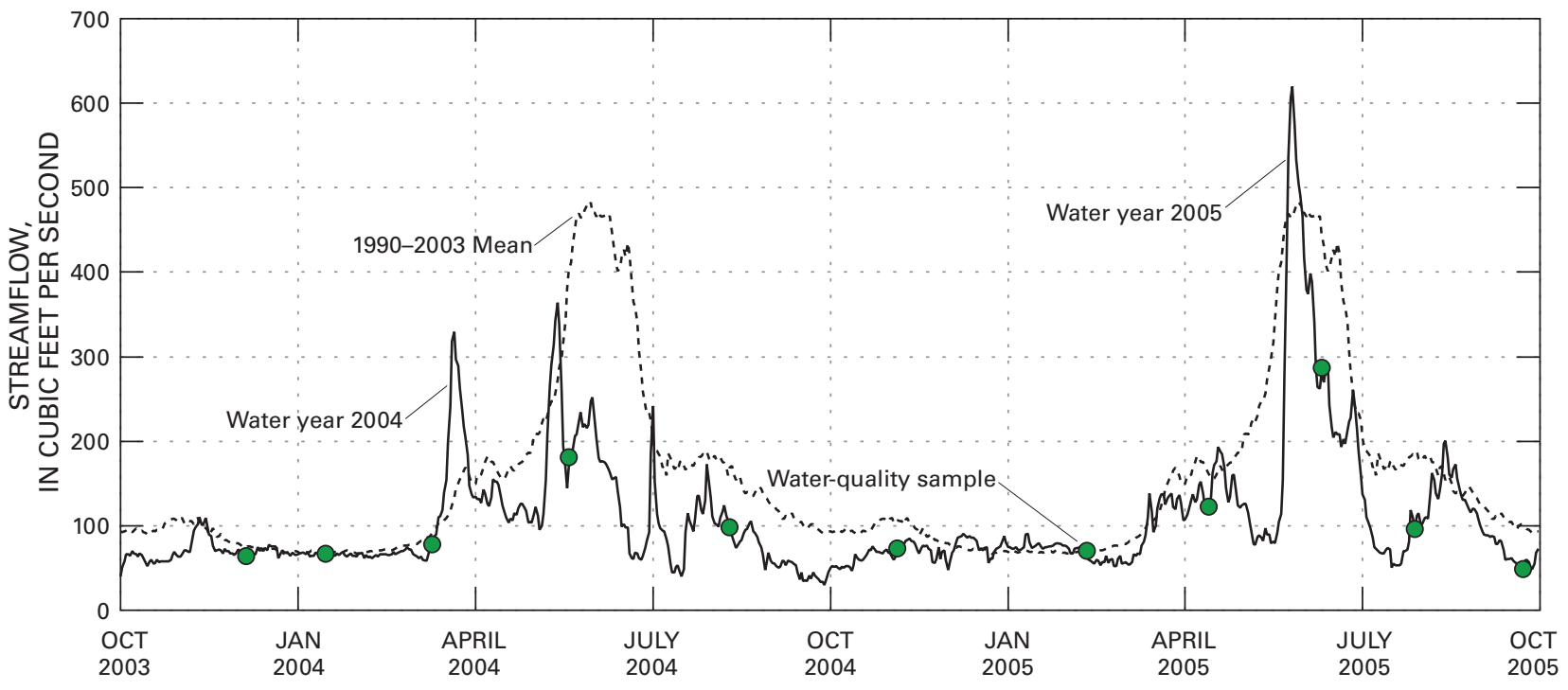

Figure 22. Streamflow and time distribution of water-quality samples for Tomichi Creek at Gunnison. 

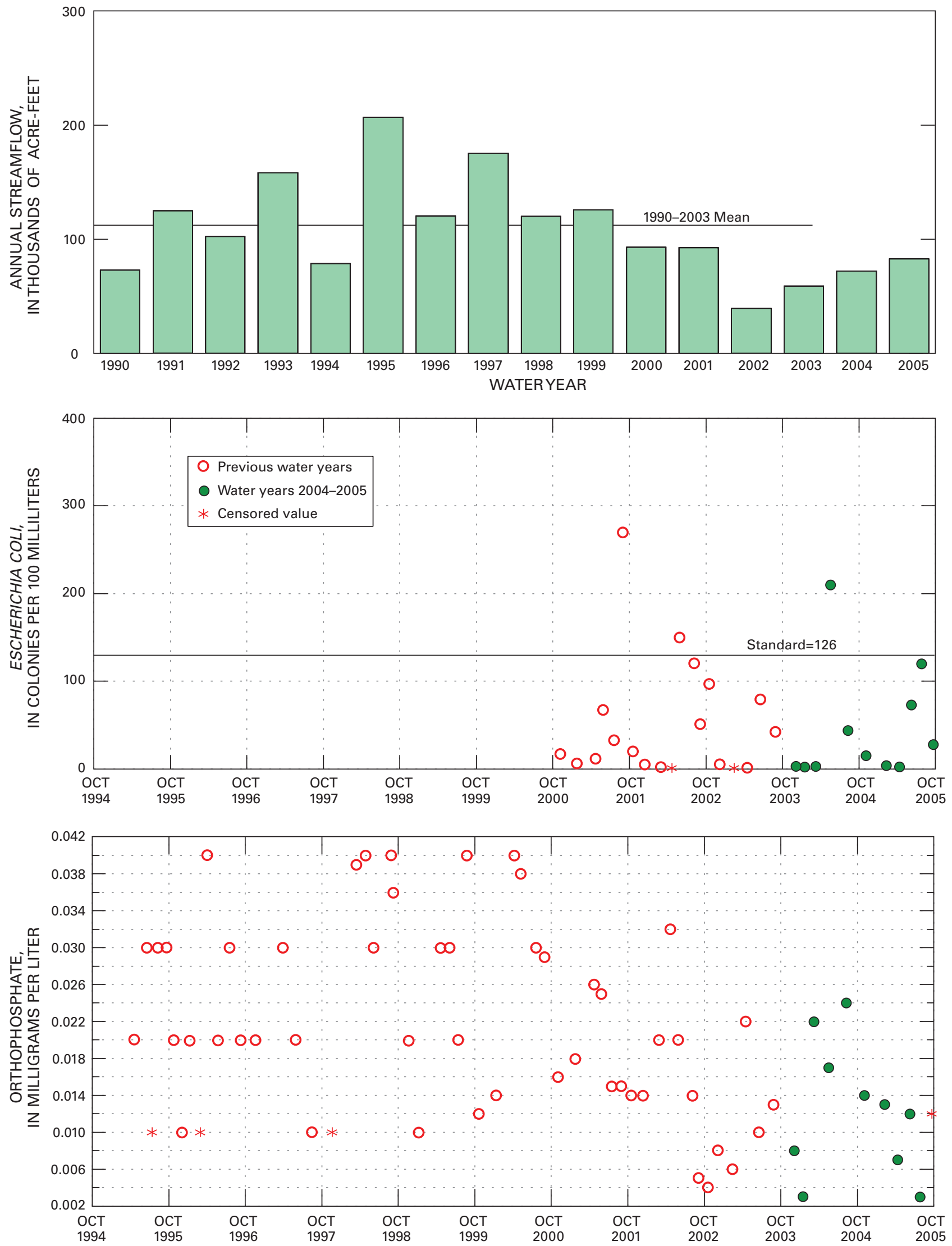

Figure 23. Annual streamflow and distribution of selected water-quality constituents relative to time for Tomichi Creek at Gunnison. 
Table 12. Summary of measured constituents and properties for Tomichi Creek at Gunnison station 09119000.

$\left[\mathrm{mg} / \mathrm{L}\right.$, milligrams per liter; $\mu \mathrm{S} / \mathrm{cm}$, microsiemens per centimeter at $25^{\circ}$ Celsius; $\mu \mathrm{g} / \mathrm{L}$, micrograms per liter; ${ }^{\circ} \mathrm{C}$, degrees Celsius; $\mathrm{CaCO}_{3}$, calcium carbonate; $\mathrm{N} / \mathrm{A}$, not applicable; $\mathrm{NTU}$, nephelometric turbidity units; (ch), chronic standard; mL, milliliters; --, no value; LRL, laboratory reporting level; <, less than; BOD, biochemical oxygen demand]

\begin{tabular}{|c|c|c|c|c|c|c|c|c|c|c|c|c|}
\hline \multirow[b]{2}{*}{$\begin{array}{l}\text { Constituent } \\
\text { or property }\end{array}$} & \multirow[b]{2}{*}{ Units } & \multirow{2}{*}{$\begin{array}{c}\text { Period } \\
\text { (water year) }\end{array}$} & \multirow{2}{*}{$\begin{array}{l}\text { Number of } \\
\text { samples }\end{array}$} & \multirow{2}{*}{$\begin{array}{l}\text { Number of } \\
\text { censored } \\
\text { values }\end{array}$} & \multirow[b]{2}{*}{ Median' } & \multicolumn{2}{|c|}{ Maximum } & \multirow{2}{*}{$\begin{array}{c}\text { 85th } \\
\text { percentile }^{1}\end{array}$} & \multicolumn{2}{|c|}{ Standard $^{2}$} & \multirow[b]{2}{*}{ Trend $^{3}$} & \multirow[b]{2}{*}{ Comment } \\
\hline & & & & & & Value & Date & & Value & $\begin{array}{c}\text { Number of } \\
\text { exceedances }\end{array}$ & & \\
\hline \multirow[t]{2}{*}{ Dissolved oxygen } & $\mathrm{mg} / \mathrm{L}$ & $2004-2005$ & 11 & 0 & 9.7 & 11.9 & $08 / 10 / 04$ & 10.7 & 6 & 0 & None & Minimum=6.0 \\
\hline & & $1995-2003$ & 54 & 0 & 9.4 & 12.1 & $11 / 20 / 97$ & & & 0 & & 15 th percentile $=7.8$ \\
\hline \multirow[t]{2}{*}{$\mathrm{pH}$} & Standard & 2004-2005 & 11 & 0 & 8.2 & 8.8 & 08/10/04 & 8.4 & $6.5-9.0$ & 0 & None & Minimum=7.4 \\
\hline & units & $1995-2003$ & 54 & 0 & 8.2 & 8.7 & ${ }^{4} 08 / 27 / 98$ & & & 0 & & 15 th percentile $=7.9$ \\
\hline \multirow[t]{2}{*}{ Specific conductance } & $\mu \mathrm{S} / \mathrm{cm}$ & 2004-2005 & 11 & 0 & 276 & 327 & 09/22/05 & 340 & None & N/A & None & \\
\hline & & $1995-2003$ & 54 & 0 & 254 & 399 & $05 / 31 / 02$ & & & N/A & & \\
\hline \multirow[t]{2}{*}{ Temperature } & ${ }^{\circ} \mathrm{C}$ & 2004-2005 & 11 & 0 & 9.6 & 19.8 & 08/10/04 & 17.0 & 20 & 0 & N/A & \\
\hline & & $1995-2003$ & 54 & 0 & 10.6 & 21.5 & $07 / 18 / 01$ & & & 6 & & \\
\hline \multirow[t]{2}{*}{ Hardness (computed) } & $\mathrm{mg} / \mathrm{L}$ & 2004-2005 & 7 & 0 & 152 & 164 & $09 / 22 / 05$ & 167 & None & N/A & $\left({ }^{5}\right)$ & \\
\hline & as $\mathrm{CaCO}_{3}$ & $1995-2003$ & 36 & 0 & 114 & 191 & $05 / 31 / 02$ & & & N/A & & \\
\hline \multirow[t]{2}{*}{ Calcium } & $\mathrm{mg} / \mathrm{L}^{3}$ & 2004-2005 & 7 & 0 & 42 & 48 & $09 / 22 / 05$ & 47 & None & N/A & $\left({ }^{5}\right)$ & \\
\hline & & $1995-2003$ & 36 & 0 & 32 & 57 & $05 / 31 / 02$ & & & N/A & & \\
\hline \multirow[t]{2}{*}{ Magnesium } & $\mathrm{mg} / \mathrm{L}$ & 2004-2005 & 7 & 0 & 11 & 12 & ${ }^{4} 05 / 19 / 04$ & 12 & None & N/A & $\left({ }^{5}\right)$ & \\
\hline & & $1995-2003$ & 36 & 0 & 8 & 13 & ${ }^{4} 08 / 07 / 02$ & & & N/A & & \\
\hline \multirow[t]{2}{*}{ Ammonia } & $\mathrm{mg} / \mathrm{L}$ & 2004-2005 & 11 & 7 & 0 & 0.018 & $02 / 10 / 05$ & 0.017 & None & N/A & $\left({ }^{5}\right)$ & Current LRL $=0.01$ \\
\hline & & $1995-2003$ & 54 & 34 & 0 & 0.050 & ${ }^{4} 04 / 01 / 96$ & & & N/A & & \\
\hline \multirow{2}{*}{$\begin{array}{l}\text { Un-ionized ammonia } \\
\text { (computed) }\end{array}$} & $\mathrm{mg} / \mathrm{L}$ & 2004-2005 & 11 & 7 & 0 & 0.00028 & 05/19/04 & 0.00036 & 0.02 & 0 & $\left({ }^{5}\right)$ & \\
\hline & & $1995-2003$ & 54 & 34 & 0 & 0.00158 & 07/19/96 & & & 0 & & \\
\hline \multirow{2}{*}{$\begin{array}{l}\text { Ammonia plus organic } \\
\text { nitrogen (total) }\end{array}$} & $\mathrm{mg} / \mathrm{L}$ & 2004-2005 & 11 & 0 & 0.34 & 0.60 & 05/19/04 & 0.53 & None & N/A & $\left({ }^{5}\right)$ & \\
\hline & & $1995-2003$ & 54 & 6 & 0.28 & 1.2 & $04 / 07 / 00$ & & & N/A & & \\
\hline \multirow[t]{2}{*}{ Nitrite plus nitrate } & $\mathrm{mg} / \mathrm{L}$ & 2004-2005 & 11 & 7 & 0 & 0.090 & $02 / 10 / 05$ & 0.060 & ${ }^{6} 10$ & 0 & $\left({ }^{5}\right)$ & Current LRL $=0.016$ \\
\hline & & $1995-2003$ & 54 & 30 & 0 & 0.106 & $01 / 12 / 00$ & & & 0 & & \\
\hline \multirow[t]{2}{*}{ Nitrite } & $\mathrm{mg} / \mathrm{L}$ & 2004-2005 & 11 & 2 & 0.001 & 0.003 & $02 / 10 / 05$ & 0.001 & 0.05 & 0 & $\left({ }^{5}\right)$ & Current LRL $=0.002$ \\
\hline & & $1995-2003$ & 54 & 43 & 0 & 0.020 & ${ }^{4} 11 / 21 / 96$ & & & 0 & & \\
\hline \multirow[t]{2}{*}{ Orthophosphate } & $\mathrm{mg} / \mathrm{L}$ & 2004-2005 & 11 & 1 & 0.012 & 0.024 & 08/10/04 & 0.030 & None & N/A & Down & Current LRL $=0.012$ \\
\hline & & $1995-2003$ & 54 & 3 & 0.020 & 0.040 & ${ }^{4} 04 / 07 / 00$ & & & N/A & & \\
\hline \multirow[t]{2}{*}{ Phosphorus (total) } & $\mathrm{mg} / \mathrm{L}$ & 2004-2005 & 11 & 0 & 0.050 & 0.121 & 03/10/04 & 0.073 & 0.1 & 1 & None & Concern \\
\hline & & 1995-2003 & 54 & 1 & 0.043 & 0.270 & 04/07/00 & & & 3 & & \\
\hline \multirow[t]{2}{*}{ Aluminum } & $\mu \mathrm{g} / \mathrm{L}$ & 2004-2005 & 7 & 0 & 2 & 2 & ${ }^{4} 05 / 19 / 04$ & 2 & 87 (ch) & 0 & $\left({ }^{5}\right)$ & \\
\hline & & 1995-2003 & 29 & 23 & 0 & 80 & 06/19/95 & & & 0 & & \\
\hline
\end{tabular}


Table 12. Summary of measured constituents and properties for Tomichi Creek at Gunnison station 09119000.—Continued

[mg/L, milligrams per liter; $\mu \mathrm{S} / \mathrm{cm}$, microsiemens per centimeter at $25^{\circ}$ Celsius; $\mu \mathrm{g} / \mathrm{L}$, micrograms per liter; ${ }^{\circ} \mathrm{C}$, degrees Celsius; $\mathrm{CaCO}_{3}$, calcium carbonate; $\mathrm{N} / \mathrm{A}$, not applicable; $\mathrm{NTU}$, nephelometric turbidity units; (ch), chronic standard; mL, milliliters; --, no value; LRL, laboratory reporting level; <, less than; BOD, biochemical oxygen demand]

\begin{tabular}{|c|c|c|c|c|c|c|c|c|c|c|c|c|}
\hline \multirow{2}{*}{$\begin{array}{l}\text { Constituent } \\
\text { or property }\end{array}$} & \multirow[b]{2}{*}{ Units } & \multirow{2}{*}{$\begin{array}{c}\text { Period } \\
\text { (water year) }\end{array}$} & \multirow{2}{*}{$\begin{array}{l}\text { Number of } \\
\text { samples }\end{array}$} & \multirow{2}{*}{$\begin{array}{c}\text { Number of } \\
\text { censored } \\
\text { values }\end{array}$} & \multirow[b]{2}{*}{ Median' } & \multicolumn{2}{|c|}{ Maximum } & \multirow{2}{*}{$\begin{array}{c}\text { 85th } \\
\text { percentile }^{1}\end{array}$} & \multicolumn{2}{|c|}{ Standard $^{2}$} & \multirow[b]{2}{*}{ Trend $^{3}$} & \multirow[b]{2}{*}{ Comment } \\
\hline & & & & & & Value & Date & & Value & $\begin{array}{c}\text { Number of } \\
\text { exceedances }\end{array}$ & & \\
\hline \multirow[t]{2}{*}{ Cadmium } & $\mu \mathrm{g} / \mathrm{L}$ & 2004-2005 & 7 & 7 & 0 & ${ }^{7}<0.04$ & -- & 0 & $2.5(\mathrm{ch})$ & 0 & $\left({ }^{5}\right)$ & Current LRL $=0.04$ \\
\hline & & $1995-2003$ & 29 & 29 & 0 & ${ }^{7}<0.1$ & -- & & & 0 & & \\
\hline \multirow[t]{2}{*}{ Copper } & $\mu \mathrm{g} / \mathrm{L}$ & 2004-2005 & 7 & 0 & 1 & 2.1 & $04 / 13 / 05$ & 1.5 & $10.0(\mathrm{ch})$ & 0 & $\left({ }^{5}\right)$ & \\
\hline & & 1995-2003 & 29 & 15 & 0 & 2.0 & ${ }^{4} 06 / 19 / 95$ & & & 0 & & \\
\hline \multirow[t]{2}{*}{ Iron } & $\mu \mathrm{g} / \mathrm{L}$ & 2004-2005 & 7 & 0 & 62 & 78 & $06 / 10 / 05$ & 110 & 300 & 0 & $\left({ }^{5}\right)$ & Water supply standard \\
\hline & & 1995-2003 & 24 & 0 & 66 & 190 & 06/19/95 & & & 0 & & \\
\hline \multirow[t]{2}{*}{ Lead } & $\mu \mathrm{g} / \mathrm{L}$ & 2004-2005 & 7 & 0 & 0.09 & 0.22 & 04/13/05 & 0.06 & $2.9(\mathrm{ch})$ & 0 & $\left({ }^{5}\right)$ & \\
\hline & & $1995-2003$ & 29 & 28 & 0 & 0.09 & 09/04/02 & & & 0 & & \\
\hline \multirow[t]{2}{*}{ Manganese } & $\mu \mathrm{g} / \mathrm{L}$ & 2004-2005 & 7 & 0 & 40 & 92 & $06 / 10 / 05$ & 85 & 1,721 & 0 & $\left({ }^{5}\right)$ & Water supply standard $=50$ \\
\hline & & $1995-2003$ & 24 & 0 & 31 & 124 & $04 / 22 / 02$ & & & 0 & & \\
\hline \multirow[t]{2}{*}{ Silver } & $\mu \mathrm{g} / \mathrm{L}$ & 2004-2005 & 7 & 7 & 0 & ${ }^{7}<0.2$ & -- & 0 & $0.40(\mathrm{ch})$ & 0 & $\left({ }^{5}\right)$ & Current LRL $=0.2$ \\
\hline & & $1995-2003$ & 17 & 17 & 0 & ${ }^{7}<0.3$ & -- & & & 0 & & \\
\hline \multirow[t]{2}{*}{ Zinc } & $\mu \mathrm{g} / \mathrm{L}$ & 2004-2005 & 7 & 0 & 0.8 & 1.0 & 06/10/05 & 0.9 & $132(\mathrm{ch})$ & 0 & $\left({ }^{5}\right)$ & \\
\hline & & 1995-2003 & 29 & 25 & 0 & 12 & 04/23/01 & & & 0 & & \\
\hline \multirow[t]{2}{*}{ Suspended sediment } & $\mathrm{mg} / \mathrm{L}$ & 2004-2005 & 7 & 0 & 6 & 17 & $04 / 13 / 05$ & 24 & None & N/A & $\left({ }^{5}\right)$ & \\
\hline & & 1995-2003 & 34 & 0 & 10 & 135 & $03 / 27 / 97$ & & & N/A & & \\
\hline \multirow[t]{2}{*}{ Turbidity } & NTU & 2004-2005 & 7 & 0 & 3.3 & 6.8 & 03/10/04 & 8.7 & None & N/A & $\left({ }^{5}\right)$ & \\
\hline & & $1995-2003$ & 13 & 0 & 3.5 & 14.0 & $05 / 29 / 01$ & & & N/A & & \\
\hline \multirow[t]{2}{*}{ Escherichia coli } & Colonies & 2004-2005 & 11 & 0 & 15 & 210 & $05 / 19 / 04$ & $\left({ }^{8}\right)$ & 126 & 1 & $\left({ }^{5}\right)$ & Geometric mean $=12$ \\
\hline & per $100 \mathrm{~mL}$ & 1995-2003 & 19 & 2 & 20 & 270 & 08/31/01 & & & 2 & & \\
\hline \multirow[t]{2}{*}{ BOD } & $\mathrm{mg} / \mathrm{L}$ & 2004-2005 & 0 & -- & -- & -- & -- & 2.4 & None & -- & $\left({ }^{5}\right)$ & \\
\hline & & $1995-2003$ & 32 & 25 & 0 & 2.7 & 04/07/00 & & & N/A & & \\
\hline
\end{tabular}

${ }^{1}$ Censored values were replaced with 0 to compute median and 85 th percentiles (coliform censored values replaced with 1). See "Definitions of Terms" section.

${ }^{2}$ Colorado Department of Public Health and Environment, classification and numeric standards for Gunnison and Lower Dolores River Basins (2006); and USEPA, Quality criteria for water (1986).

${ }^{3}$ Period of record for trend analysis is water year 1996-water year 2005; flow-adjusted seasonal Kendall method, Helsel and Hirsch, Statistical Methods in Water Resources (1993).

${ }^{4}$ Multiple dates for maximum.

${ }^{5}$ Statistic cannot be computed due to number of censored values or insufficient data.

${ }^{6}$ Instream standard for nitrate.

${ }^{7}$ All values censored, current LRL used for maximum.

${ }^{8}$ Use geometric mean for comparison to standard. 


\section{Gunnison River at County Road 32 below Gunnison, C0}

Current Reason for Inclusion: This station is downstream from the City of Gunnison treatment plant discharge. When compared to sites Gunnison River at Gunnison and Tomichi Creek at Gunnison, this site should give insight to the change in water quality due to point and nonpoint sources in the City of Gunnison. Characterize water-quality conditions upstream from Curecanti National Recreation Area. Long-term monitoring.

General Station Information:

Location: County Road 32 bridge, 0.25 mile south of U.S. Highway 50, and 3.3 miles west of Gunnison.

Station Type: USGS water quality

Latitude: 383103

Drainage area: $2,128 \mathrm{mi}^{2}$

HUC: 14020002

Longitude: 1065942

Stream segment: 14

\section{USGS Data Summary:}

Period of Record:

Water quality: December 1994 to September 2005

General Chemistry:

Water type: Calcium carbonate

Hardness: Moderately hard

pH: Low concern

Dissolved oxygen: Low concern

Nutrients:

Total phosphorus: Concern

E. coli: Low concern

Trace Elements/Metals: Low concern

Other constituents of concern: None

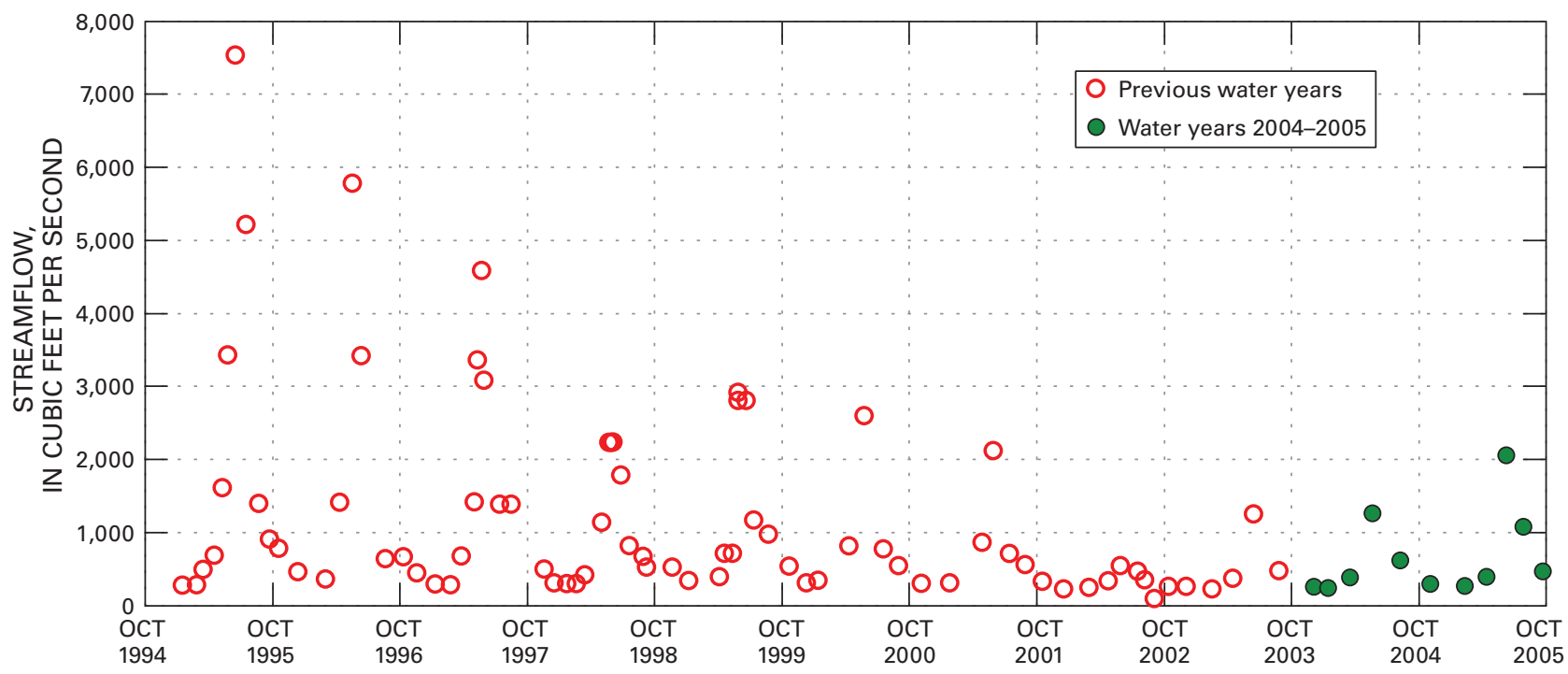

Figure 24. Time distribution and streamflow of water-quality samples for Gunnison River at County Road 32 below Gunnison. 

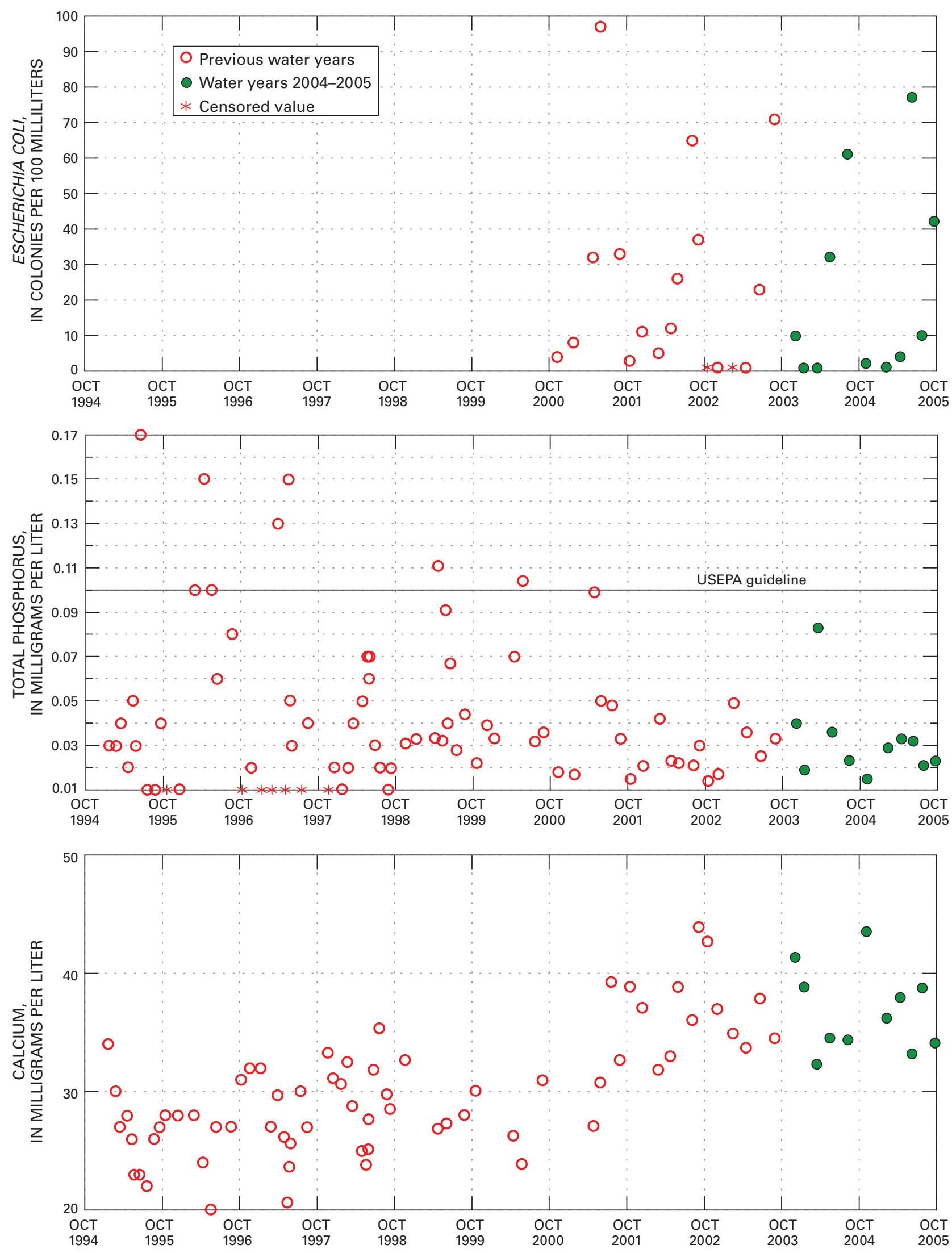

Figure 25. Distribution of selected water-quality constituents relative to time for Gunnison River at County Road 32 below Gunnison. 
Table 13. Summary of measured constituents and properties for Gunnison River at County Road 32 station 383103106594200.

$\left[\mathrm{mg} / \mathrm{L}\right.$, milligrams per liter; $\mu \mathrm{S} / \mathrm{cm}$, microsiemens per centimeter at $25^{\circ}$ Celsius; $\mu \mathrm{g} / \mathrm{L}$, micrograms per liter; ${ }^{\circ} \mathrm{C}$, degrees Celsius; $\mathrm{CaCO}_{3}$, calcium carbonate; $\mathrm{N} / \mathrm{A}$, not applicable; $\mathrm{NTU}$, nephelometric turbidity units; (ch), chronic standard; mL, milliliters; --, no value; LRL, laboratory reporting level; <, less than; BOD, biological oxygen demand]

\begin{tabular}{|c|c|c|c|c|c|c|c|c|c|c|c|c|}
\hline \multirow{2}{*}{$\begin{array}{l}\text { Constituent } \\
\text { or property }\end{array}$} & \multirow[b]{2}{*}{ Units } & \multirow{2}{*}{$\begin{array}{c}\text { Period } \\
\text { (water year) }\end{array}$} & \multirow{2}{*}{$\begin{array}{l}\text { Number of } \\
\text { samples }\end{array}$} & \multirow{2}{*}{$\begin{array}{c}\text { Number of } \\
\text { censored } \\
\text { values }\end{array}$} & \multirow{2}{*}{ Median' } & \multicolumn{2}{|c|}{ Maximum } & \multirow{2}{*}{$\begin{array}{c}\text { 85th } \\
\text { percentile }^{1}\end{array}$} & \multicolumn{2}{|c|}{ Standard $^{2}$} & \multirow[b]{2}{*}{ Trend $^{3}$} & \multirow[b]{2}{*}{ Comment } \\
\hline & & & & & & Value & Date & & Value & $\begin{array}{c}\text { Number of } \\
\text { exceedances }\end{array}$ & & \\
\hline \multirow[t]{2}{*}{ Dissolved oxygen } & $\mathrm{mg} / \mathrm{L}$ & 2004-2005 & 11 & 0 & 9.5 & 12.8 & $02 / 10 / 05$ & 11.2 & 6 & 0 & None & Minimum=7.0 \\
\hline & & 1995-2003 & 77 & 0 & 9.3 & 12.5 & $12 / 17 / 97$ & & & 0 & & 15 th percentile $=8.3$ \\
\hline \multirow[t]{2}{*}{$\mathrm{pH}$} & Standard & 2004-2005 & 11 & 0 & 8.5 & 8.8 & $02 / 10 / 05$ & 8.5 & $6.5-9.0$ & 0 & None & Minimum=7.6 \\
\hline & Units & 1995-2003 & 77 & 0 & 8.2 & 8.6 & ${ }^{4} 01 / 19 / 95$ & & & 0 & & 15 th percentile $=8.0$ \\
\hline \multirow[t]{2}{*}{ Specific conductance } & $\mu \mathrm{S} / \mathrm{cm}$ & 2004-2005 & 11 & 0 & 232 & 276 & $04 / 13 / 05$ & 255 & None & N/A & Up & \\
\hline & & 1995-2003 & 78 & 0 & 224 & 288 & 09/05/02 & & & N/A & & \\
\hline \multirow[t]{2}{*}{ Temperature } & ${ }^{\circ} \mathrm{C}$ & 2004-2005 & 11 & 0 & 9.8 & 14.5 & $05 / 19 / 04$ & 14.4 & 20 & 0 & N/A & \\
\hline & & 1995-2003 & 78 & 0 & 7.6 & 17.3 & 07/20/98 & & & 0 & & \\
\hline \multirow[t]{2}{*}{ Hardness (computed) } & $\mathrm{mg} / \mathrm{L}$ & 2004-2005 & 11 & 0 & 123 & 145 & $11 / 04 / 04$ & 126 & None & N/A & Up & \\
\hline & as $\mathrm{CaCO}_{3}$ & 1995-2003 & 66 & 0 & 100 & 147 & $09 / 05 / 02$ & & & N/A & & \\
\hline \multirow[t]{2}{*}{ Calcium } & $\mathrm{mg} / \mathrm{L}$ & 2004-2005 & 11 & 0 & 36 & 44 & $11 / 04 / 04$ & 37 & None & N/A & Up & \\
\hline & & 1995-2003 & 66 & 0 & 29 & 44 & 09/05/02 & & & N/A & & \\
\hline \multirow[t]{2}{*}{ Magnesium } & $\mathrm{mg} / \mathrm{L}$ & 2004-2005 & 11 & 0 & 7.9 & 9.0 & $11 / 04 / 04$ & 7.9 & None & N/A & Up & \\
\hline & & 1995-2003 & 66 & 0 & 6.5 & 9.1 & 09/05/02 & & & N/A & & \\
\hline \multirow[t]{2}{*}{ Ammonia } & $\mathrm{mg} / \mathrm{L}$ & 2004-2005 & 11 & 5 & 0.005 & 0.008 & ${ }^{4} 04 / 13 / 05$ & 0.020 & None & N/A & $\left({ }^{5}\right)$ & Current LRL $=0.01$ \\
\hline & & $1995-2003$ & 77 & 45 & 0 & 0.070 & $05 / 22 / 98$ & & & N/A & & \\
\hline \multirow{2}{*}{$\begin{array}{l}\text { Un-ionized ammonia } \\
\text { (computed) }\end{array}$} & $\mathrm{mg} / \mathrm{L}$ & 2004-2005 & 11 & 5 & 0.0001 & 0.00040 & $02 / 10 / 05$ & 0.00032 & 0.02 & 0 & $\left({ }^{5}\right)$ & \\
\hline & & 1995-2003 & 77 & 45 & 0 & 0.00317 & $09 / 09 / 98$ & & & 0 & & \\
\hline \multirow{2}{*}{$\begin{array}{l}\text { Ammonia plus organic } \\
\text { nitrogen (total) }\end{array}$} & $\mathrm{mg} / \mathrm{L}$ & 2004-2005 & 11 & 0 & 0.20 & 0.43 & 03/17/04 & 0.35 & None & N/A & $\left({ }^{5}\right)$ & \\
\hline & & 1995-2003 & 77 & 19 & 0.18 & 0.60 & ${ }^{4} 06 / 19 / 95$ & & & N/A & & \\
\hline \multirow[t]{2}{*}{ Nitrite plus nitrate } & $\mathrm{mg} / \mathrm{L}$ & 2004-2005 & 11 & 1 & 0.038 & 0.127 & $01 / 15 / 05$ & 0.104 & ${ }^{6} 10$ & 0 & $\left({ }^{5}\right)$ & Current LRL $=0.016$ \\
\hline & & 1995-2003 & 77 & 12 & 0.060 & 0.178 & 03/01/02 & & & 0 & & \\
\hline \multirow[t]{2}{*}{ Nitrite } & $\mathrm{mg} / \mathrm{L}$ & 2004-2005 & 11 & 2 & 0.001 & 0.002 & ${ }^{4} 02 / 10 / 05$ & 0.002 & 0.05 & 0 & $\left({ }^{5}\right)$ & Current LRL $=0.002$ \\
\hline & & $1995-2003$ & 77 & 48 & 0 & 0.020 & ${ }^{4} 06 / 13 / 96$ & & & 0 & & \\
\hline \multirow[t]{2}{*}{ Orthophosphate } & $\mathrm{mg} / \mathrm{L}$ & 2004-2005 & 11 & 3 & 0.005 & 0.027 & $03 / 17 / 04$ & 0.020 & None & N/A & $\left({ }^{5}\right)$ & Current LRL $=0.006$ \\
\hline & & 1995-2003 & 77 & 10 & 0.010 & 0.030 & ${ }^{4} 02 / 20 / 98$ & & & N/A & & \\
\hline \multirow[t]{2}{*}{ Phosphorus (total) } & $\mathrm{mg} / \mathrm{L}$ & 2004-2005 & 11 & 0 & 0.029 & 0.083 & 03/17/04 & 0.070 & 0.1 & 0 & None & Concern \\
\hline & & 1995-2003 & 77 & 7 & 0.032 & 0.170 & 06/16/95 & & & 6 & & \\
\hline
\end{tabular}


Table 13. Summary of measured constituents and properties for Gunnison River at County Road 32 station 383103106594200.-Continued

[mg/L, milligrams per liter; $\mu \mathrm{S} / \mathrm{cm}$, microsiemens per centimeter at $25^{\circ}$ Celsius; $\mu \mathrm{g} / \mathrm{L}$, micrograms per liter; ${ }^{\circ} \mathrm{C}$, degrees Celsius; $\mathrm{CaCO}_{3}$, calcium carbonate; $\mathrm{N} / \mathrm{A}$, not applicable; $\mathrm{NTU}$, nephelometric turbidity units; (ch), chronic standard; mL, milliliters; --, no value; LRL, laboratory reporting level; <, less than; BOD, biological oxygen demand]

\begin{tabular}{|c|c|c|c|c|c|c|c|c|c|c|c|c|}
\hline \multirow[b]{2}{*}{$\begin{array}{l}\text { Constituent } \\
\text { or property }\end{array}$} & \multirow[b]{2}{*}{ Units } & \multirow{2}{*}{$\begin{array}{c}\text { Period } \\
\text { (water year) }\end{array}$} & \multirow[b]{2}{*}{$\begin{array}{l}\text { Number of } \\
\text { samples }\end{array}$} & \multirow{2}{*}{$\begin{array}{l}\text { Number of } \\
\text { censored } \\
\text { values }\end{array}$} & \multirow[b]{2}{*}{ Median' ${ }^{1}$} & \multicolumn{2}{|c|}{ Maximum } & \multirow{2}{*}{$\begin{array}{c}\text { 85th } \\
\text { percentile }^{1}\end{array}$} & \multicolumn{2}{|c|}{ Standard $^{2}$} & \multirow[b]{2}{*}{ Trend $^{3}$} & \multirow[b]{2}{*}{ Comment } \\
\hline & & & & & & Value & Date & & Value & $\begin{array}{c}\text { Number of } \\
\text { exceedances }\end{array}$ & & \\
\hline \multirow[t]{2}{*}{ Selenium } & $\mu \mathrm{g} / \mathrm{L}$ & $2004-2005$ & 11 & 9 & 0 & 0.3 & $01 / 15 / 04$ & 0.3 & $4.6(\mathrm{ch})$ & 00 & $\left({ }^{5}\right)$ & \\
\hline & & 2001-2003 & 16 & 11 & 0 & 0.5 & $04 / 25 / 02$ & & & 00 & & \\
\hline \multirow[t]{2}{*}{ Cadmium } & $\mu \mathrm{g} / \mathrm{L}$ & 2004-2005 & 11 & 9 & 0 & 0.03 & ${ }^{4} 06 / 09 / 05$ & 0 & $2.4(\mathrm{ch})$ & 0 & $\left({ }^{5}\right)$ & Current LRL $=0.04$ \\
\hline & & $1995-2003$ & 34 & 33 & 0 & 0.02 & 06/19/03 & & & 0 & & \\
\hline \multirow[t]{2}{*}{ Copper } & $\mu \mathrm{g} / \mathrm{L}$ & 2004-2005 & 11 & 0 & 0.9 & 1.8 & $06 / 09 / 05$ & 1.0 & $9.6(\mathrm{ch})$ & 0 & $\left({ }^{5}\right)$ & \\
\hline & & $1995-2003$ & 34 & 17 & 0.2 & 1.9 & 06/01/98 & & & 0 & & \\
\hline \multirow[t]{2}{*}{ Lead } & $\mu \mathrm{g} / \mathrm{L}$ & 2004-2005 & 11 & 6 & 0 & 0.2 & $03 / 17 / 04$ & 0.07 & $2.7(\mathrm{ch})$ & 0 & $\left({ }^{5}\right)$ & Current LRL $=0.08$ \\
\hline & & $1995-2003$ & 34 & 29 & 0 & 3.0 & 08/07/02 & & & 1 & & \\
\hline \multirow[t]{2}{*}{ Manganese } & $\mu \mathrm{g} / \mathrm{L}$ & 2004-2005 & 11 & 0 & 15 & 36 & $03 / 17 / 04$ & 21 & 1,691 & 0 & None & Water supply standard $=50$ \\
\hline & & $1995-2003$ & 66 & 0 & 15 & 38 & $03 / 16 / 95$ & & & 0 & & \\
\hline \multirow[t]{2}{*}{ Silver } & $\mu \mathrm{g} / \mathrm{L}$ & 2004-2005 & 11 & 11 & 0 & ${ }^{7}<0.2$ & -- & 0 & $0.36(\mathrm{ch})$ & 0 & $\left({ }^{5}\right)$ & Current LRL $=0.2$ \\
\hline & & 1999-2003 & 26 & 26 & 0 & ${ }^{7}<0.2$ & -- & & & 0 & & \\
\hline \multirow[t]{2}{*}{ Zinc } & $\mu \mathrm{g} / \mathrm{L}$ & 2004-2005 & 11 & 0 & 1.4 & 8.6 & $06 / 09 / 05$ & 5.0 & $123(\mathrm{ch})$ & 0 & $\left({ }^{5}\right)$ & \\
\hline & & $1995-2003$ & 34 & 20 & 0 & 16 & $04 / 27 / 01$ & & & 0 & & \\
\hline \multirow[t]{2}{*}{ Turbidity } & NTU & 2004-2005 & 11 & 0 & 2.8 & 4.5 & 03/17/04 & 5.0 & None & N/A & $\left({ }^{5}\right)$ & \\
\hline & & $2001-2003$ & 17 & 0 & 3.7 & 14.0 & $04 / 27 / 01$ & & & N/A & & \\
\hline \multirow[t]{2}{*}{ Escherichia coli } & Colonies & 2004-2005 & 11 & 0 & 10 & 77 & $06 / 09 / 05$ & $\left({ }^{8}\right)$ & 126 & 0 & $\left({ }^{5}\right)$ & Geometric mean $=8$ \\
\hline & per $100 \mathrm{~mL}$ & 2001-2003 & 18 & 2 & 12 & 97 & $05 / 31 / 01$ & & & 0 & & \\
\hline \multirow[t]{2}{*}{ BOD } & $\mathrm{mg} / \mathrm{L}$ & 2004-2005 & 11 & 8 & 0 & 2.6 & ${ }^{4} 02 / 10 / 05$ & 1.5 & None & N/A & $\left({ }^{5}\right)$ & \\
\hline & & 1995-2003 & 29 & 25 & 0 & 2.8 & 03/01/02 & & & N/A & & \\
\hline
\end{tabular}

${ }^{1}$ Censored values were replaced with 0 to compute median and 85 th percentiles (coliform censored values replaced with 1). See "Definitions of Terms" section.

${ }^{2}$ Colorado Department of Public Health and Environment, classification and numeric standards for Gunnison and Lower Dolores River Basins (2006) and USEPA, Quality criteria for water (1986).

${ }^{3}$ Period of record for trend analysis is water year 1996-water year 2005; flow-adjusted seasonal Kendall method, Helsel and Hirsch, Statistical Methods in Water Resources (1993).

${ }^{4}$ Multiple dates for maximum.

${ }^{5}$ Statistic cannot be computed due to number of censored values or insufficient data.

${ }^{6}$ Instream standard for nitrate.

${ }^{7}$ All values censored, current LRL used for maximum.

${ }^{8}$ Use geometric mean for comparison to standard. 


\section{Henson Creek at Mouth at Lake City}

Current Reason for Inclusion: To collect baseline data, characterize effects from mining, and facilitate remediation of the affected surface waters.

General Station Information:

Location: Highway 149 road bridge over Henson Creek in Lake City.

Station Type: USGS water quality

Latitude: 380133

Longitude: 1070900
Drainage area: $83 \mathrm{mi}^{2}$

Stream segment: 30

HUC: 14020002

\section{USGS Data Summary:}

Period of Record:

Water quality: December 2003 to September 2005

General Chemistry:

Water type: Insufficient major-ion data

pH: Low concern

Dissolved oxygen: Low concern

Nutrients: Low concern

Trace Elements/Metals:

Aluminum and zinc: Concern

Other constituents of concern: None

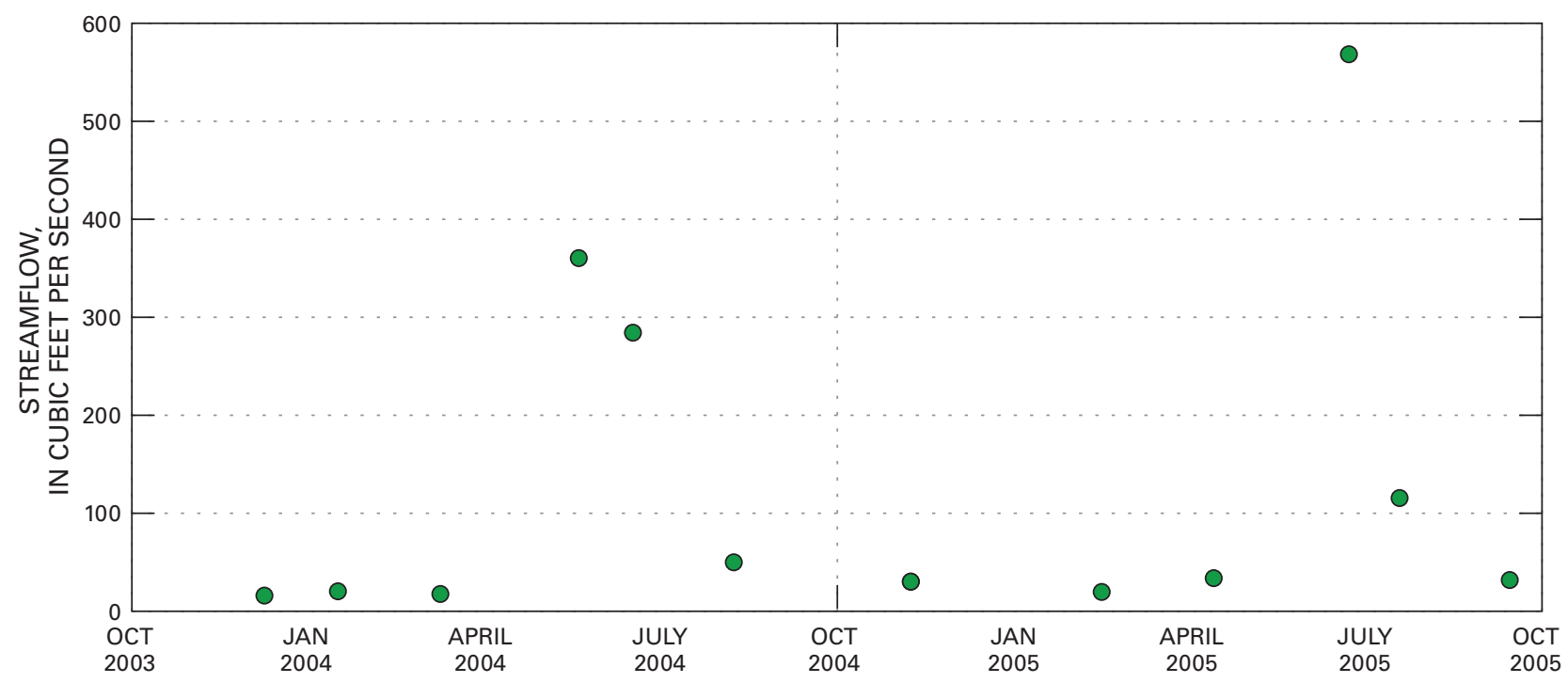

Figure 26. Time distribution and streamflow of water-quality samples for Henson Creek at mouth at Lake City. 

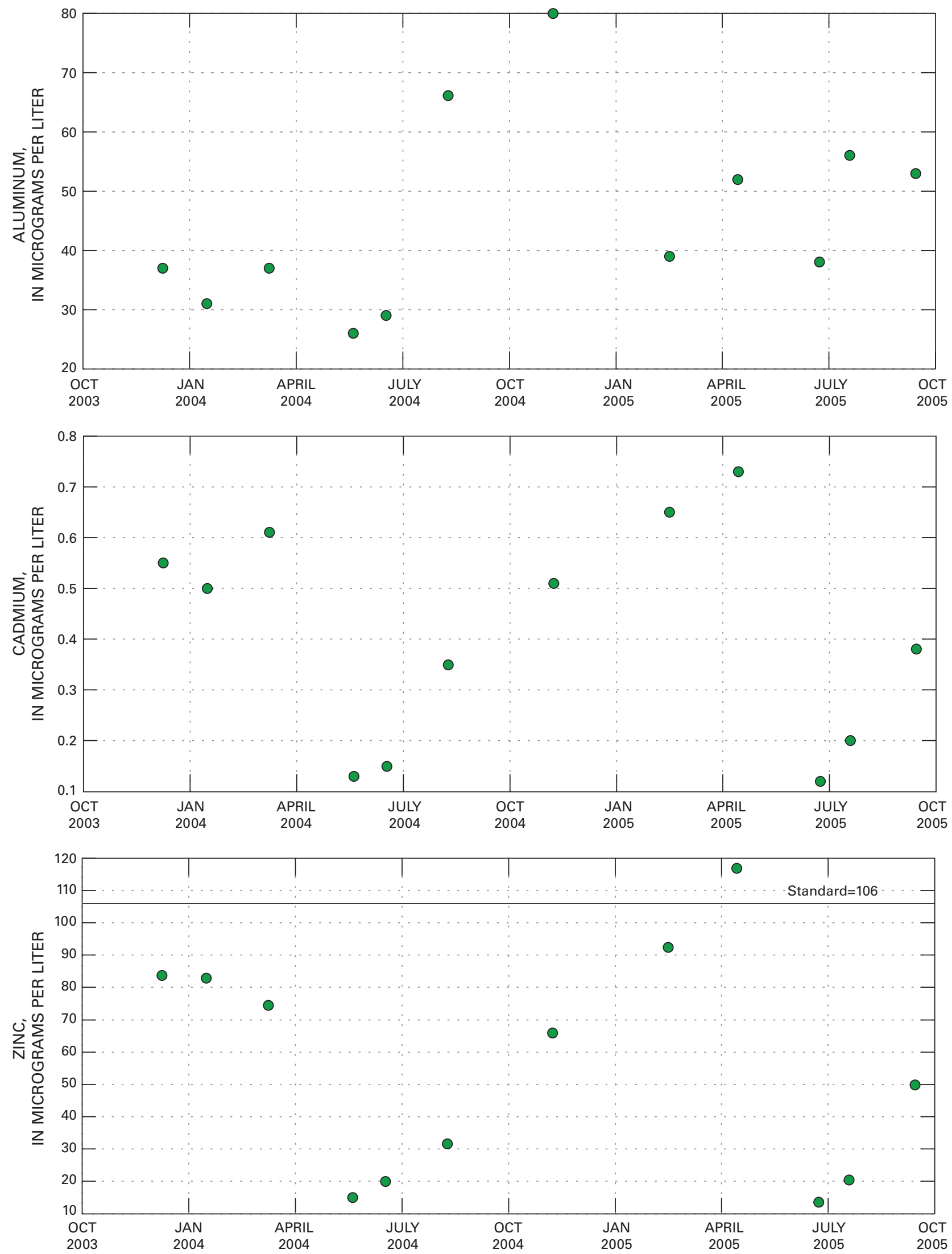

Figure 27. Distribution of selected water-quality constituents relative to time for Henson Creek at mouth at Lake City. 
Table 14. Summary of measured constituents and properties for Henson Creek at Mouth at Lake City station 380133107190000.

$\left[\mathrm{mg} / \mathrm{L}\right.$, milligrams per liter; $\mu \mathrm{S} / \mathrm{cm}$, microsiemens per centimeter at $25^{\circ}$ Celsius; $\mu \mathrm{g} / \mathrm{L}$, micrograms per liter; ${ }^{\circ} \mathrm{C}$, degrees Celsius; $\mathrm{CaCO}_{3}$, calcium carbonate; $\mathrm{N} / \mathrm{A}$, not applicable; $\mathrm{NTU}$, nephelometric turbidity units; (ch), chronic standard; mL, milliliters; --, no value; LRL, laboratory reporting level; <, less than]

\begin{tabular}{|c|c|c|c|c|c|c|c|c|c|c|c|c|}
\hline \multirow[b]{2}{*}{$\begin{array}{l}\text { Constituent } \\
\text { or property }\end{array}$} & \multirow[b]{2}{*}{ Units } & \multirow[b]{2}{*}{$\begin{array}{c}\text { Period } \\
\text { (water year) }\end{array}$} & \multirow{2}{*}{$\begin{array}{l}\text { Number of } \\
\text { samples }\end{array}$} & \multirow{2}{*}{$\begin{array}{c}\text { Number of } \\
\text { censored } \\
\text { values }\end{array}$} & \multirow{2}{*}{ Median' } & \multicolumn{2}{|c|}{ Maximum } & \multirow{2}{*}{$\begin{array}{c}\text { 85th } \\
\text { percentile }^{1}\end{array}$} & \multicolumn{2}{|c|}{ Standard $^{2}$} & \multirow[b]{2}{*}{ Trend $^{3}$} & \multirow[b]{2}{*}{ Comment } \\
\hline & & & & & & Value & Date & & Value & $\begin{array}{c}\text { Number of } \\
\text { exceedances }\end{array}$ & & \\
\hline Dissolved oxygen & $\mathrm{mg} / \mathrm{L}$ & 2004-2005 & 12 & 0 & 9.8 & 10.7 & $12 / 09 / 03$ & 10.5 & 6 & 0 & $\left({ }^{5}\right)$ & $\begin{array}{l}\text { Minimum }=7.7 \\
15 \text { th percentile }=8.3\end{array}$ \\
\hline $\mathrm{pH}$ & $\begin{array}{l}\text { Standard } \\
\text { units }\end{array}$ & 2004-2005 & 12 & 0 & 7.7 & 8.4 & 03/09/04 & 8.0 & $6.5-9.0$ & 0 & $\left({ }^{5}\right)$ & $\begin{array}{l}\text { Minimum }=7.1 \\
15 \text { th percentile }=7.4\end{array}$ \\
\hline Specific conductance & $\mu \mathrm{S} / \mathrm{cm}$ & 2004-2005 & 12 & 0 & 194 & 220 & $02 / 15 / 05$ & 216 & None & N/A & $\left({ }^{5}\right)$ & \\
\hline Temperature & ${ }^{\circ} \mathrm{C}$ & 2004-2005 & 12 & 0 & 2 & 14.9 & $07 / 19 / 05$ & 13.5 & 20 & 0 & N/A & \\
\hline Hardness (computed) & $\begin{array}{c}\mathrm{mg} / \mathrm{L} \\
\text { as } \mathrm{CaCO}_{3}\end{array}$ & 2004-2005 & 12 & 0 & 86 & 98 & $01 / 16 / 04$ & 98 & None & N/A & $\left({ }^{5}\right)$ & \\
\hline Calcium & $\mathrm{mg} / \mathrm{L}$ & 2004-2005 & 12 & 0 & 30 & 34 & $02 / 15 / 05$ & 34 & None & N/A & $\left({ }^{5}\right)$ & \\
\hline Magnesium & $\mathrm{mg} / \mathrm{L}$ & 2004-2005 & 12 & 0 & 2.9 & 3.6 & 03/09/04 & 3.5 & None & N/A & $\left({ }^{5}\right)$ & \\
\hline Ammonia & $\mathrm{mg} / \mathrm{L}$ & 2004-2005 & 12 & 10 & 0 & 0.013 & $07 / 19 / 05$ & 0.006 & None & N/A & $\left({ }^{5}\right)$ & Current LRL $=0.01$ \\
\hline $\begin{array}{l}\text { Un-ionized ammonia } \\
\text { (computed) }\end{array}$ & $\mathrm{mg} / \mathrm{L}$ & 2004-2005 & 12 & 10 & 0 & 0.00022 & $07 / 19 / 05$ & 0.00005 & 0.02 & 0 & $\left({ }^{5}\right)$ & \\
\hline $\begin{array}{l}\text { Ammonia plus organic } \\
\text { nitrogen (total) }\end{array}$ & $\mathrm{mg} / \mathrm{L}$ & 2004-2005 & 12 & 4 & 0.06 & 0.20 & $05 / 20 / 04$ & 0.09 & None & N/A & $\left({ }^{5}\right)$ & Current LRL $=0.01$ \\
\hline Nitrite plus nitrate & $\mathrm{mg} / \mathrm{L}$ & 2004-2005 & 12 & 0 & 0.116 & 0.184 & $05 / 20 / 04$ & 0.16 & ${ }^{6} 10$ & 0 & $\left({ }^{5}\right)$ & \\
\hline Nitrite & $\mathrm{mg} / \mathrm{L}$ & 2004-2005 & 12 & 6 & 0.0005 & 0.001 & ${ }^{4} 09 / 14 / 05$ & 0.001 & 0.05 & 0 & $\left({ }^{5}\right)$ & Current LRL $=0.002$ \\
\hline Orthophosphate & $\mathrm{mg} / \mathrm{L}$ & 2004-2005 & 12 & 12 & 0 & ${ }^{7}<0.006$ & -- & 0 & None & N/A & $\left({ }^{5}\right)$ & Current LRL $=0.006$ \\
\hline Phosphorus (total) & $\mathrm{mg} / \mathrm{L}$ & 2004-2005 & 12 & 0 & 0.012 & 0.088 & $05 / 20 / 04$ & 0.045 & 0.1 & 0 & $(5)$ & \\
\hline Aluminum & $\mu \mathrm{g} / \mathrm{L}$ & 2004-2005 & 12 & 0 & 38 & 80 & $11 / 08 / 04$ & 66 & 87 (ch) & 0 & $\left({ }^{5}\right)$ & Concern \\
\hline Cadmium & $\mu \mathrm{g} / \mathrm{L}$ & 2004-2005 & 12 & 0 & 0.44 & 0.73 & $04 / 14 / 05$ & 0.65 & $2.0(\mathrm{ch})$ & 0 & $\left(5^{5}\right)$ & \\
\hline Copper & $\mu \mathrm{g} / \mathrm{L}$ & 2004-2005 & 12 & 0 & 1.9 & 2.7 & $04 / 14 / 05$ & 2.3 & $8.0(\mathrm{ch})$ & 0 & $\left({ }^{5}\right)$ & \\
\hline Iron & $\mu \mathrm{g} / \mathrm{L}$ & 2004-2005 & 12 & 2 & 5.5 & 22 & 08/09/04 & 18 & 300 (WS) & & $\left({ }^{5}\right)$ & Current LRL $=6$ \\
\hline Lead & $\mu \mathrm{g} / \mathrm{L}$ & 2004-2005 & 12 & 0 & 0.20 & 0.56 & 08/09/04 & 0.30 & $2.2(\mathrm{ch})$ & 0 & $\left({ }^{5}\right)$ & \\
\hline Manganese & $\mu \mathrm{g} / \mathrm{L}$ & 2004-2005 & 12 & 0 & 30 & 48 & $04 / 14 / 05$ & 47 & 1,583 & 0 & $\left({ }^{5}\right)$ & Water supply standard $=50$ \\
\hline Silver & $\mu \mathrm{g} / \mathrm{L}$ & 2004-2005 & 12 & 12 & 0 & ${ }^{7}<0.2$ & -- & 0 & $0.26(\mathrm{ch})$ & 0 & $\left({ }^{5}\right)$ & Current LRL $=0.2$ \\
\hline Zinc & $\mu \mathrm{g} / \mathrm{L}$ & 2004-2005 & 12 & 0 & 58 & 117 & $04 / 14 / 05$ & 92 & $106(\mathrm{ch})$ & 1 & $\left({ }^{5}\right)$ & Concern \\
\hline Turbidity & NTU & 2004-2005 & 12 & 4 & 2.7 & 15 & $05 / 20 / 04$ & 7.9 & None & N/A & $(5)$ & Current LRL $=2$ \\
\hline Sediment & $\mathrm{mg} / \mathrm{L}$ & 2004-2005 & 12 & 0 & 4.5 & 79 & $05 / 20 / 04$ & 36 & None & N/A & $\left({ }^{5}\right)$ & \\
\hline
\end{tabular}

${ }^{1}$ Censored values were replaced with 0 to compute median and 85 th percentiles (coliform censored values replaced with 1). See "Definitions of Terms" section.

${ }^{2}$ Colorado Department of Public Health and Environment, classification and numeric standards for Gunnison and Lower Dolores River Basins (2006) and USEPA, Quality criteria for water (1986).

${ }^{3}$ Period of record for trend analysis is water year 1996-water year 2005; flow-adjusted seasonal Kendall method, Helsel and Hirsch, Statistical Methods in Water Resources (1993).

${ }^{4}$ Multiple dates for maximum.

${ }^{5}$ Statistic cannot be computed due to number of censored values or insufficient data.

${ }^{6}$ Instream standard for nitrate.

${ }^{7}$ All values censored, current LRL used for maximum. 


\section{Lake Fork Gunnison River near Lake City}

Current Reason for Inclusion: To collect baseline data, characterize effects from mining, and facilitate remediation of the affected surface waters.

\section{General Station Information:}

Location: Private road bridge, 1/8 mile east of Hwy 149, and 1.1 miles northeast of Lake City.

Station Type: USGS water quality

Latitude: 380233

Longitude: 1071840
Drainage area: Not determined Stream segment: 29
HUC: 14020002

\section{USGS Data Summary:}

Period of Record:

Water quality: December 2003 to September 2005

General Chemistry:

Water type: Insufficient major-ion data

pH: Low concern

Dissolved oxygen: Low concern

Nutrients: Low concern

E. coli: Low concern

Trace Elements/Metals:

Aluminum: Concern

Other constituents of concern: None

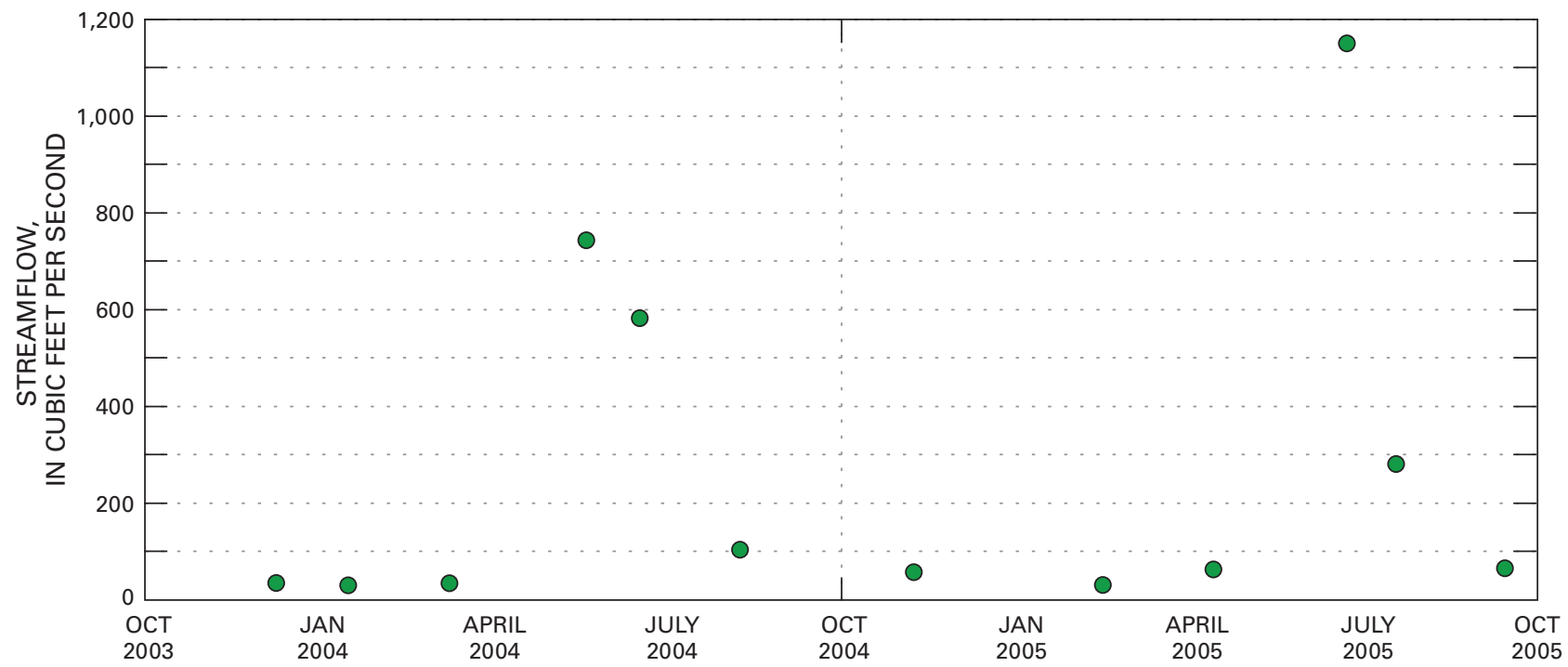

Figure 28. Time distribution and streamflow of water-quality samples for Lake Fork Gunnison River near Lake City. 

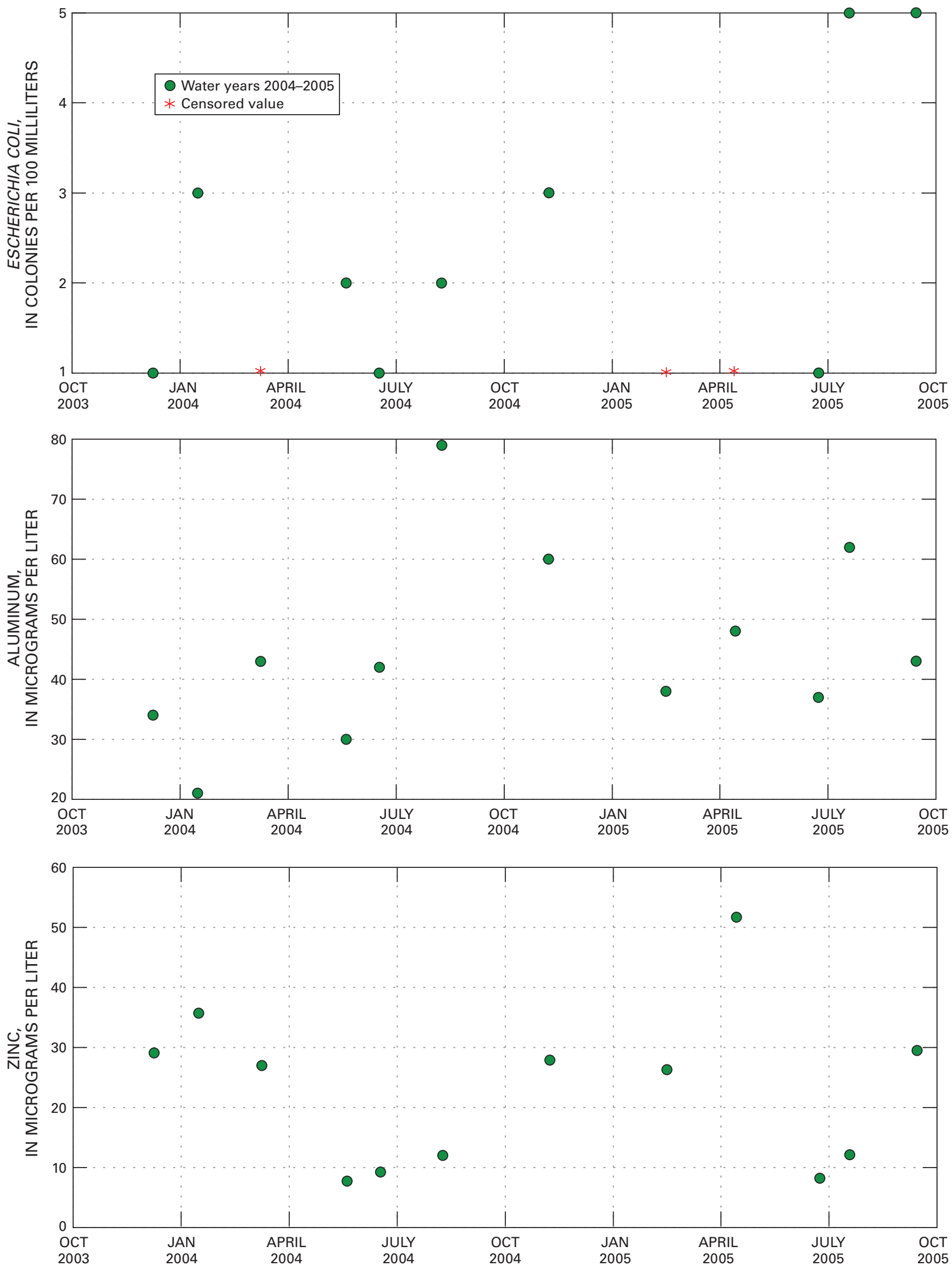

Figure 29. Distribution of selected water-quality constituents relative to time for Lake Fork Gunnison River near Lake City. 
Table 15. Summary of measured constituents and properties for Lake Fork Gunnison River near Lake City station 380233107180701.

$\left[\mathrm{mg} / \mathrm{L}\right.$, milligrams per liter; $\mu \mathrm{S} / \mathrm{cm}$, microsiemens per centimeter at $25^{\circ}$ Celsius; $\mu \mathrm{g} / \mathrm{L}$, micrograms per liter; ${ }^{\circ} \mathrm{C}$, degrees Celsius; $\mathrm{CaCO}_{3}$, calcium carbonate; $\mathrm{N} / \mathrm{A}$, not applicable; $\mathrm{NTU}$, nephelometric turbidity units; (ch), chronic standard; mL, milliliters; --, no value; LRL, laboratory reporting level; <, less than]

\begin{tabular}{|c|c|c|c|c|c|c|c|c|c|c|c|c|}
\hline \multirow[b]{2}{*}{$\begin{array}{l}\text { Constituent } \\
\text { or property }\end{array}$} & \multirow[b]{2}{*}{ Units } & \multirow[b]{2}{*}{$\begin{array}{c}\text { Period } \\
\text { (water year) }\end{array}$} & \multirow[b]{2}{*}{$\begin{array}{c}\text { Number of } \\
\text { samples }\end{array}$} & \multirow{2}{*}{$\begin{array}{c}\text { Number of } \\
\text { censored } \\
\text { values }\end{array}$} & \multirow[b]{2}{*}{ Median' } & \multicolumn{2}{|c|}{ Maximum } & \multirow[b]{2}{*}{$\begin{array}{c}\text { 85th } \\
\text { percentile }^{1}\end{array}$} & \multicolumn{2}{|c|}{ Standard $^{2}$} & \multirow[b]{2}{*}{ Trend $^{3}$} & \multirow[b]{2}{*}{ Comment } \\
\hline & & & & & & Value & Date & & Value & $\begin{array}{c}\text { Number of } \\
\text { exceedances }\end{array}$ & & \\
\hline Dissolved oxygen & $\mathrm{mg} / \mathrm{L}$ & 2004-2005 & 12 & 0 & 9.0 & 10.4 & ${ }^{4} 02 / 15 / 05$ & 10.4 & 6 & 0 & $\left({ }^{5}\right)$ & $\begin{array}{l}\text { Minimum }=7.6 \\
15 \text { th percentile }=7.6\end{array}$ \\
\hline $\mathrm{pH}$ & $\begin{array}{l}\text { Standard } \\
\text { Units }\end{array}$ & 2004-2005 & 12 & 0 & 8.0 & 8.4 & 03/09/04 & 8.2 & $6.5-9.0$ & 0 & $\left({ }^{5}\right)$ & $\begin{array}{l}\text { Minimum }=7.6 \\
15 \text { th percentile }=7.8\end{array}$ \\
\hline Specific conductance & $\mu \mathrm{S} / \mathrm{cm}$ & 2004-2005 & 12 & 0 & 187 & 218 & $03 / 09 / 04$ & 203 & None & N/A & $\left({ }^{5}\right)$ & \\
\hline Temperature & ${ }^{\circ} \mathrm{C}$ & 2004-2005 & 12 & 0 & 6.2 & 17.5 & 08/09/04 & 16.7 & 20 & 0 & N/A & \\
\hline $\begin{array}{l}\text { Hardness } \\
\text { (computed) }\end{array}$ & $\begin{array}{c}\mathrm{mg} / \mathrm{L} \\
\text { as } \mathrm{CaCO}_{3}\end{array}$ & 2004-2005 & 12 & 0 & 81 & 90 & ${ }^{4} 03 / 09 / 04$ & 90 & None & N/A & $\left({ }^{5}\right)$ & \\
\hline Calcium & $\mathrm{mg} / \mathrm{L}^{3}$ & 2004-2005 & 12 & 0 & 28 & 30 & ${ }^{4} 01 / 16 / 04$ & 30 & None & N/A & $\left({ }^{5}\right)$ & \\
\hline Magnesium & $\mathrm{mg} / \mathrm{L}$ & 2004-2005 & 12 & 0 & 3.0 & 3.8 & 03/09/04 & 3.6 & None & N/A & (5) & \\
\hline Ammonia & $\mathrm{mg} / \mathrm{L}$ & 2004-2005 & 12 & 5 & 0.008 & 0.039 & 01/16/04 & 0.031 & None & N/A & $\left({ }^{5}\right)$ & Current LRL $=0.01$ \\
\hline $\begin{array}{l}\text { Un-ionized ammonia } \\
\text { (computed) }\end{array}$ & $\mathrm{mg} / \mathrm{L}$ & 2004-2005 & 12 & 5 & 0.00007 & 0.00104 & 08/09/04 & 0.00082 & 0.02 & 0 & $\left({ }^{5}\right)$ & \\
\hline $\begin{array}{l}\text { Ammonia plus organic } \\
\text { nitrogen (total) }\end{array}$ & $\mathrm{mg} / \mathrm{L}$ & 2004-2005 & 12 & 1 & 0.08 & 0.17 & $03 / 09 / 04$ & 0.16 & None & N/A & $\left({ }^{5}\right)$ & Current LRL $=0.01$ \\
\hline Nitrite plus nitrate & $\mathrm{mg} / \mathrm{L}$ & 2004-2005 & 12 & 0 & 0.059 & 0.109 & $05 / 20 / 04$ & 0.102 & ${ }^{6} 10$ & 0 & $\left({ }^{5}\right)$ & \\
\hline Nitrite & $\mathrm{mg} / \mathrm{L}$ & 2004-2005 & 12 & 5 & 0.001 & 0.005 & $09 / 14 / 05$ & 0.003 & 0.05 & 0 & $\left({ }^{5}\right)$ & Current LRL $=0.002$ \\
\hline Orthophosphate & $\mathrm{mg} / \mathrm{L}$ & 2004-2005 & 12 & 11 & 0 & 0.003 & 08/09/04 & 0 & None & N/A & $\left({ }^{5}\right)$ & Current LRL $=0.006$ \\
\hline Phosphorus (total) & $\mathrm{mg} / \mathrm{L}$ & 2004-2005 & 12 & 0 & 0.013 & 0.047 & $05 / 20 / 04$ & 0.030 & 0.1 & 0 & $\left({ }^{5}\right)$ & \\
\hline Aluminum & $\mu \mathrm{g} / \mathrm{L}$ & 2004-2005 & 12 & 0 & 42 & 79 & 08/09/04 & 62 & 87 (ch) & 0 & $\left({ }^{5}\right)$ & Concern \\
\hline Cadmium & $\mu \mathrm{g} / \mathrm{L}$ & 2004-2005 & 12 & 0 & 0.20 & 0.36 & $04 / 14 / 05$ & 0.26 & $1.9(\mathrm{ch})$ & 0 & $\left({ }^{5}\right)$ & \\
\hline Copper & $\mu \mathrm{g} / \mathrm{L}$ & 2004-2005 & 12 & 0 & 1.6 & 2.0 & $04 / 14 / 05$ & 1.9 & $7.6(\mathrm{ch})$ & 0 & $\left({ }^{5}\right)$ & \\
\hline Iron & $\mu \mathrm{g} / \mathrm{L}$ & $2004-2005$ & 12 & 1 & 8.5 & 20 & 08/09/04 & 15 & 300 (WS) & & $(5)$ & Current LRL $=6$ \\
\hline Lead & $\mu \mathrm{g} / \mathrm{L}$ & 2004-2005 & 12 & 1 & 0.18 & 0.33 & 08/09/04 & 0.28 & $2.0(\mathrm{ch})$ & 0 & $\left({ }^{5}\right)$ & Current LRL $=0.08$ \\
\hline Manganese & $\mu \mathrm{g} / \mathrm{L}$ & 2004-2005 & 12 & 0 & 25 & 42 & $05 / 20 / 04$ & 33 & 1,543 & 0 & $(5)$ & Water supply standard $=50$ \\
\hline Silver & $\mu \mathrm{g} / \mathrm{L}$ & 2004-2005 & 12 & 12 & 0 & ${ }^{7}<0.2$ & -- & 0 & $0.23(\mathrm{ch})$ & 0 & $\left({ }^{5}\right)$ & Current LRL $=0.2$ \\
\hline Zinc & $\mu \mathrm{g} / \mathrm{L}$ & 2004-2005 & 12 & 0 & 27 & 52 & $04 / 14 / 05$ & 36 & $100(\mathrm{ch})$ & 1 & $\left({ }^{5}\right)$ & \\
\hline Escherichia coli & $\begin{array}{l}\text { Colonies } \\
\text { per } 100 \mathrm{~mL}\end{array}$ & 2004-2005 & 12 & 3 & 1.5 & 5 & ${ }^{4} 07 / 19 / 05$ & $\left({ }^{8}\right)$ & 126 & 0 & $(5)$ & Geometric mean $=2$ \\
\hline Turbidity & NTU & 2004-2005 & 12 & 5 & 2.1 & 6.8 & $05 / 20 / 04$ & 4.5 & None & N/A & $\left({ }^{5}\right)$ & Current LRL $=2$ \\
\hline Sediment & $\mathrm{mg} / \mathrm{L}$ & 2004-2005 & 12 & 0 & 2.5 & 116 & $06 / 17 / 04$ & 35 & None & N/A & $\left(5^{5}\right)$ & \\
\hline
\end{tabular}

${ }^{1}$ Censored values were replaced with 0 to compute median and 85th percentiles (coliform censored values replaced with 1). See "Definitions of Terms" section.

${ }^{2}$ Colorado Department of Public Health and Environment, classification and numeric standards for Gunnison and Lower Dolores River Basins (2006) and USEPA, Quality criteria for water (1986).

${ }^{3}$ Period of record for trend analysis is water year 1996-water year 2005; flow-adjusted seasonal Kendall method, Helsel and Hirsch, Statistical Methods in Water Resources (1993).

${ }^{4}$ Multiple dates for maximum.

${ }^{5}$ Statistic cannot be computed due to number of censored values or insufficient data.

${ }^{6}$ Instream standard for nitrate.

${ }^{7}$ All values censored, current LRL used for maximum.

${ }^{8}$ Use geometric mean for comparison to standard. 


\section{Crested Butte Wastewater Treatment Plant}

Current Reason for Inclusion: Describe phosphorus concentrations.

\section{General Station Information:}

Station Type: USGS water quality

\section{USGS Data Summary:}

Period of Record:

Water quality: December 2001 to September 2005

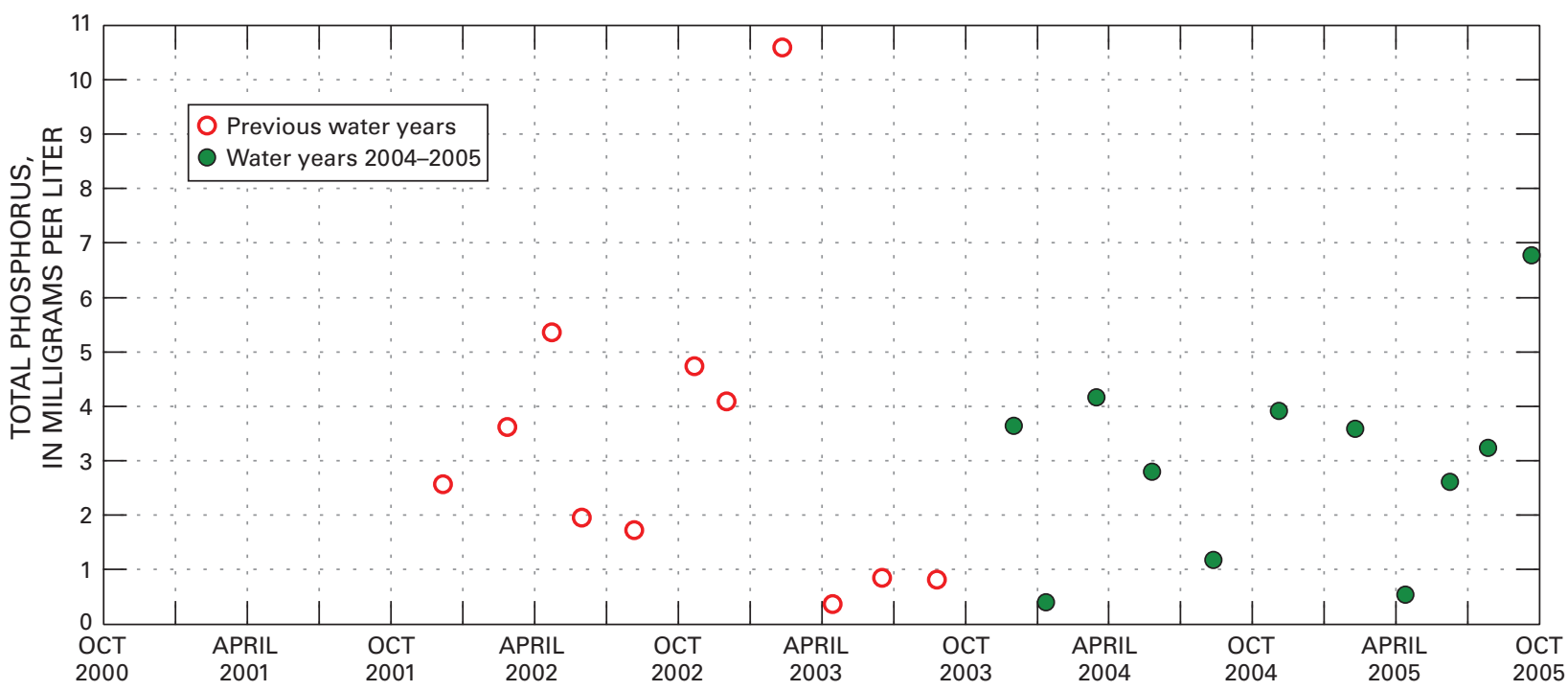

Figure 30. Distribution of total phosphorus samples relative to time for Crested Butte Wastewater Treatment Plant.

Table 16. Summary of total phosphorus for Crested Butte Wastewater Treatment Plant site 385229106583100.

[mg/L, milligrams per liter; N/A, not applicable]

\begin{tabular}{|c|c|c|c|c|c|c|c|c|c|c|c|}
\hline \multirow{2}{*}{$\begin{array}{l}\text { Constituent } \\
\text { or property }\end{array}$} & \multirow[b]{2}{*}{ Units } & \multirow{2}{*}{$\begin{array}{c}\text { Period } \\
\text { (water year) }\end{array}$} & \multirow{2}{*}{$\begin{array}{l}\text { Number of } \\
\text { samples }\end{array}$} & \multirow{2}{*}{$\begin{array}{c}\text { Number of } \\
\text { censored } \\
\text { values }\end{array}$} & \multirow[b]{2}{*}{ Median' } & \multicolumn{2}{|c|}{ Maximum } & \multirow{2}{*}{$\begin{array}{c}\text { 85th } \\
\text { percentile }^{1}\end{array}$} & \multicolumn{2}{|r|}{ Standard } & \multirow[b]{2}{*}{ Trend $^{2}$} \\
\hline & & & & & & Value & Date & & Value & $\begin{array}{c}\text { Number of } \\
\text { exceedances }\end{array}$ & \\
\hline Phosphorus (total) & $\mathrm{mg} / \mathrm{L}$ & 2004-2005 & 11 & 0 & 3.2 & 6.8 & $09 / 20 / 05$ & 4.8 & N/A & N/A & $\left({ }^{2}\right)$ \\
\hline
\end{tabular}

${ }^{1}$ Censored values were replaced with 0 to compute median and 85 th percentiles (coliform censored values replaced with 1 ). See definitions section.

${ }^{2}$ Statistic cannot be computed due to number of censored values or insufficient data. 


\section{References}

Biggs, B.J.F., 1996, Patterns in benthic algae of streams, chap. 2, in Stevenson, R. J., Bothwell, M.L., and Lowe, R.L., Algal ecology_Freshwater benthic ecosystems: San Diego, Academic Press, Harcourt Brace and Company, p. 31-56.

Colorado Department of Public Health and Environment, Water Quality Control Commission, 2006, Classification and numeric standards for Gunnison and lower Dolores River Basins, Regulation No. 35: Colorado Department of Public Health and Environment, Water Quality Control Commission [variously paginated].

Gurdak, J.J., Greve, A.I., and Spahr, N.E., 2002, Water-quality data analysis of the Upper Gunnison River Watershed, Colorado, 1989-99: U.S. Geological Survey Water-Resources Investigations Report 2002-4001, 61 p.

Helsel, D.R., and Hirsch, R.M, 1993, Statistical methods in water resources: New York, Elsevier Science Publishers B.V., 529 p.

Hem, J.D., 1992, Study and interpretation of the chemical characteristics of natural waters: U.S. Geological Survey WaterSupply Paper 2254, 263 p.

U.S. Environmental Protection Agency, 1986, Quality criteria for water, 1986: U.S. Environmental Protection Agency Report 440/5-86-001, variously paginated.

U.S. Environmental Protection Agency, 2000, Drinking water standards and health advisories: U.S. Environmental Protection Agency Report 822-B-00-001, 12 p.

U.S. Geological Survey, variously dated, National field manual for the collection of water-quality data: U.S. Geological Survey Techniques of Water-Resources Investigations, book 9, chaps. A1-A9, available online at http://pubs.water.usgs.gov/twri9A.

For more information concerning this publication, contact:

Director, Colorado Water Science Center

U.S. Geological Survey

Box 25046, MS 415

Denver Federal Center

Lakewood, Colorado 80225

(303) 236-5900

Or visit the Colorado Water Science Center Web site at:

http://co.water.usgs.gov/

This publication is available online at:

http://pubs.usgs.gov/ds/331

Publishing support provided by:

Denver Publishing Service Center, Denver, Colorado

Manuscript approved for publication, February 22, 2008

Edited by Mary A. Kidd

Graphics and layout by Joy Monson 
言

韋

$\frac{\overline{0}}{\omega}$

8

产.

을

은

产

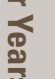

ััธ

言

I

음.

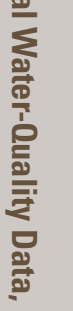

듬

D

.

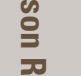

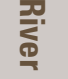

实

.

$\delta$

을

Printed on recycled paper 